\title{
Geometry and BMS Lie algebras of spatially isotropic homogeneous spacetimes
}

\author{
José Figueroa-O'Farrill, ${ }^{a}$ Ross Grassie ${ }^{a}$ and Stefan Prohazka ${ }^{b}$ \\ ${ }^{a}$ Maxwell Institute and School of Mathematics, The University of Edinburgh, \\ James Clerk Maxwell Building, Peter Guthrie Tait Road, Edinburgh EH9 3FD, Scotland, U.K. \\ ${ }^{b}$ Université Libre de Bruxelles and International Solvay Institutes, \\ Physique Mathématique des Interactions Fondamentales, \\ Campus Plaine - CP 231, B-1050 Bruxelles, Belgium, Europe \\ E-mail: j.m.figueroa@ed.ac.uk, s1131494@sms.ed.ac.uk, \\ stefan.prohazka@ulb.ac.be
}

ABSTRACT: Simply-connected homogeneous spacetimes for kinematical and aristotelian Lie algebras (with space isotropy) have recently been classified in all dimensions. In this paper, we continue the study of these "maximally symmetric" spacetimes by investigating their local geometry. For each such spacetime and relative to exponential coordinates, we calculate the (infinitesimal) action of the kinematical symmetries, paying particular attention to the action of the boosts, showing in almost all cases that they act with generic non-compact orbits. We also calculate the soldering form, the associated vielbein and any invariant aristotelian, galilean or carrollian structures. The (conformal) symmetries of the galilean and carrollian structures we determine are typically infinite-dimensional and reminiscent of BMS Lie algebras. We also determine the space of invariant affine connections on each homogeneous spacetime and work out their torsion and curvature.

Keywords: Space-Time Symmetries, Differential and Algebraic Geometry

ARXIV EPRINT: 1905.00034

Amelie Prohazka gewidmet. 


\section{Contents}

1 Introduction $\quad 2$

2 Homogeneous kinematical spacetimes 4

2.1 Classification 5

2.2 Geometric limits $\quad 7$

2.3 Geometrical properties 9

3 Local geometry of homogeneous spacetimes $\quad 11$

$\begin{array}{lll}3.1 & \text { Exponential coordinates } & 11\end{array}$

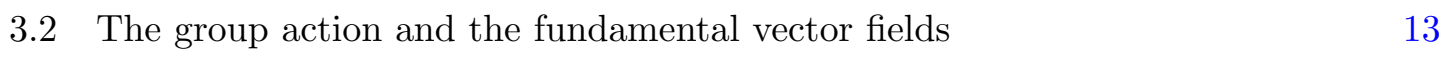

$\begin{array}{ll}3.3 \text { The action of the rotations } & 15\end{array}$

$\begin{array}{ll}3.4 & \text { The action of the boosts } \\ & 16\end{array}$

$\begin{array}{ll}3.5 & \text { Invariant connections } \\ \end{array}$

3.6 The soldering form and the canonical connection 20

$\begin{array}{lll}3.7 & \text { Invariant tensors } & 21\end{array}$

4 Invariant connections, curvature, and torsion for reductive spacetimes 22

4.1 Nomizu maps for lorentzian spacetimes 22

$\begin{array}{lll}4.1 .1 & D \geq 4 & 23\end{array}$

$\begin{array}{lll}4.1 .2 D & D=3 & 23\end{array}$

$\begin{array}{lll}4.1 .3 \quad D=2 & 23\end{array}$

$\begin{array}{lll}\text { 4.1.4 } D=1 & 23\end{array}$

4.2 Nomizu maps for riemannian spacetimes 24

$\begin{array}{lll}4.3 & \text { Nomizu maps for galilean spacetimes } & 24\end{array}$

$\begin{array}{lll}\text { 4.3.1 } & \text { Galilean spacetime }(G) & 24\end{array}$

$\begin{array}{lll}\text { 4.3.2 Galilean de Sitter spacetime (dSG) } & 25\end{array}$

4.3.3 Galilean anti de Sitter spacetime (AdSG) 25

4.3.4 Torsional galilean de Sitter spacetime $\left(\mathrm{dSG}_{\gamma=1}\right) \quad 25$

4.3.5 Torsional galilean de Sitter spacetime $\left(\mathrm{dSG}_{\gamma \neq 1}\right) \quad 26$

4.3.6 Torsional galilean anti de Sitter spacetime $\left(\right.$ AdSG $\left._{\chi}\right) \quad 27$

$\begin{array}{lll}\text { 4.3.7 Spacetime } \mathrm{S} 12_{\gamma, \chi} & 27\end{array}$

4.4 Nomizu maps for carrollian spacetimes 28

$\begin{array}{lll}\text { 4.4.1 Carrollian spacetimes (C) } & 28\end{array}$

4.4.2 (Anti) de Sitter carrollian spacetimes (dSC and AdSC) 28

$\begin{array}{lll}\text { 4.4.3 Carrollian light cone (LC) } & 29\end{array}$

$\begin{array}{lll}4.5 & \text { Nomizu maps for exotic two-dimensional spacetimes } & 29\end{array}$

4.6 Nomizu maps for aristotelian spacetimes 30

$\begin{array}{lll}\text { 4.6.1 Static spacetime (S) } & 30\end{array}$

$\begin{array}{lll}\text { 4.6.2 } & \text { Torsional static spacetime (TS) } & 32\end{array}$

4.6.3 Aristotelian spacetime A23 $\varepsilon_{\varepsilon} \quad 33$ 
5 Pseudo-riemannian spacetimes and their limits $\quad 34$

$\begin{array}{lll}5.1 & \text { Invariant structures } & 35\end{array}$

$\begin{array}{lll}\text { 5.1.1 Lorentzian and riemannian case } & 36\end{array}$

5.1.2 Non- and ultra-relativistic limits 36

5.2 Action of the boosts 36

$\begin{array}{lll}\text { 5.2.1 Lorentzian boosts } & 37\end{array}$

$\begin{array}{ll}5.2 .2 \text { Euclidean "boosts" } & 38\end{array}$

$\begin{array}{lll}5.2 .3 & \text { Galilean boosts } & 38\end{array}$

5.2.4 Carrollian boosts 38

$\begin{array}{lll}5.3 & \text { Fundamental vector fields } & 39\end{array}$

$\begin{array}{ll}5.4 & \text { Soldering form and connection one-form }\end{array} 42$

5.5 Flat limit, Minkowski (M) and euclidean spacetime (E) 43

5.6 Galilean spacetime $(\mathrm{G}) \quad 43$

$\begin{array}{lll}5.7 & \text { Carrollian spacetime (C) } & 43\end{array}$

5.8 Non-relativistic limit 44

5.9 Galilean de Sitter spacetime (dSG) 44

5.10 Galilean anti de Sitter spacetime (AdSG) 45

$\begin{array}{ll}5.11 \text { Ultra-relativistic limit } & 46\end{array}$

5.12 (Anti) de Sitter carrollian spacetimes (dSC and AdSC) 46

6 Torsional galilean spacetimes $\quad 48$

6.1 Torsional galilean de Sitter spacetime $\left(\mathrm{dSG}_{\gamma \neq 1}\right) \quad 48$

$\begin{array}{lll}\text { 6.1.1 Fundamental vector fields } & 48\end{array}$

$\begin{array}{ll}\text { 6.1.2 Soldering form and canonical connection } & 50\end{array}$

6.2 Torsional galilean de Sitter spacetime $\left(\mathrm{dSG}_{\gamma=1}\right) \quad 50$

6.2.1 Fundamental vector fields 51

6.2.2 Soldering form and canonical connection 52

6.3 Torsional galilean anti de Sitter spacetime $\left(\right.$ AdSG $\left._{\chi}\right)$

$\begin{array}{lll}\text { 6.3.1 Fundamental vector fields } & 53\end{array}$

6.3.2 Soldering form and canonical connection $\quad 54$

$\begin{array}{ll}6.4 & \text { Spacetime } \mathrm{S} 12_{\gamma, \chi}\end{array} 55$

6.4.1 Fundamental vector fields $\quad 55$

$\begin{array}{ll}\text { 6.4.2 Soldering form and canonical connection } & 57\end{array}$

$\begin{array}{lll}\text { 6.5 The action of the boosts } & 58\end{array}$

7 Carrollian light cone (LC) $\quad 62$

$\begin{array}{lll}7.1 & \text { Action of the boosts } & 62\end{array}$

$\begin{array}{lll}7.2 & \text { Fundamental vector fields } & 63\end{array}$

$\begin{array}{ll}7.3 & \text { Soldering form and canonical connection }\end{array}$ 
8 Exotic two-dimensional spacetimes $\quad 65$

$\begin{array}{lll}8.1 & \text { Spacetime S17 } & 66\end{array}$

$\begin{array}{ll}8.2 & \text { Spacetime S18 }\end{array}$

$\begin{array}{ll}8.3 & \text { Spacetime } \mathrm{S} 19 \chi\end{array}$

$\begin{array}{lll}8.4 & \text { Spacetime S20 } & 67\end{array}$

9 Aristotelian spacetimes $\quad \mathbf{6 7}$

$\begin{array}{lll}9.1 & \text { Static spacetime (S) } & 68\end{array}$

9.2 Torsional static spacetime (TS) 68

$\begin{array}{lll}\text { 9.2.1 } & \text { Fundamental vector fields } & 68\end{array}$

$\begin{array}{ll}\text { 9.2.2 Soldering form and canonical connection } & 68\end{array}$

$\begin{array}{lll}9.3 & \text { Aristotelian spacetime A23 } & 69\end{array}$

$\begin{array}{lll}\text { 9.3.1 Fundamental vector fields } & 69\end{array}$

$\begin{array}{ll}\text { 9.3.2 Soldering form and canonical connection } & 70\end{array}$

$\begin{array}{lll}9.4 & \text { Aristotelian spacetime A24 } & 70\end{array}$

$\begin{array}{lll}\text { 9.4.1 Fundamental vector fields } & 70\end{array}$

$\begin{array}{ll}\text { 9.4.2 Soldering form and canonical connection } & 71\end{array}$

10 Symmetries of the spacetime structure $\quad 71$

10.1 Symmetries of the carrollian structure (C) 72

10.2 Symmetries of the (anti) de Sitter carrollian structure (dSC and AdSC) $\quad 75$

10.3 Symmetries of the carrollian light cone (LC) 78

$\begin{array}{ll}10.4 \text { Symmetries of galilean structures } & 80\end{array}$

11 Conclusions $\quad 82$

A Modified exponential coordinates $\quad 84$

$\begin{array}{lll}\text { A.1 Carrollian spacetimes } & 84\end{array}$

A.1.1 Carrollian (anti) de Sitter spacetimes $\quad 84$

A.2 Galilean spacetimes $\quad 85$

$\begin{array}{lll}\text { A.2.1 Galilean spacetime } & 87\end{array}$

$\begin{array}{lll}\text { A.2.2 Galilean de Sitter spacetime } & 87\end{array}$

$\begin{array}{lll}\text { A.2.3 Torsional galilean de Sitter spacetime } & 87\end{array}$

$\begin{array}{lll}\text { A.2.4 Galilean anti de Sitter spacetime } & 88\end{array}$

$\begin{array}{lll}\text { A.2.5 Torsional galilean anti de Sitter spacetime } & 88\end{array}$

$\begin{array}{lll}\text { A.2.6 Spacetime } \mathrm{S} 12_{\gamma, \chi} & 88\end{array}$

$\begin{array}{ll}\text { B Conformal Killing vectors in low dimension } & 89\end{array}$ 


\section{Introduction}

Half a century ago, Bacry and Lévy-Leblond [1] asked what were the possible kinematics. They provided an answer to this question by classifying kinematical Lie algebras in $3+1$ dimensions subject to the assumptions of invariance under parity and time-reversal. They also showed that the kinematical Lie algebras in their classification could be related by contractions. Moreover they observed that each such Lie algebra acts transitively on some $(3+1)$-dimensional spatially isotropic homogeneous spacetime and that the contractions could be interpreted as geometric limits of the corresponding spacetimes. Physically, we can understand these limits as approximations and this interpretation explains why these particular spacetimes are relevant and continue to show up in different corners of physics.

Indeed, most of the spacetimes in their work are known to play a fundamental rôle in physics. For example, the de Sitter spacetime is important for cosmology, the anti de Sitter spacetime currently drives much of our understanding of quantum gravity due to the AdS/CFT correspondence [2], and, in the limit where the cosmological constant goes to zero, Minkowski spacetime is fundamental in particle physics. Other important spacetimes of this type include the galilean spacetime, which is the playing field for condensed matter systems, and the carrollian spacetime, whose relation to Bondi-Metzner-Sachs (BMS) symmetries, as shown in [3], is leading to exciting progress in our understanding of infrared physics in asymptotically flat spaces (for reviews see $[4,5]){ }^{1}$

Twenty years later, Bacry and Nuyts [18] dropped the "by no means compelling" assumptions of parity and time-reversal invariance and hence classified all kinematical Lie algebras in $3+1$ dimensions, observing that once again each such Lie algebra acts transitively on some $(3+1)$-dimensional homogeneous spacetime.

Strictly speaking, what was shown in $[1,18]$ is that every kinematical Lie algebra $\mathfrak{k}$ in their classification has a Lie subalgebra $\mathfrak{h}$ spanned by the infinitesimal generators of rotations and boosts. This suggests the existence of Lie groups $\mathcal{H} \subset \mathcal{K}$ with Lie algebras $\mathfrak{h} \subset \mathfrak{k}$ and hence of a homogeneous spacetime $\mathcal{K} / \mathcal{H}$. However the very existence of the homogeneous spacetime and its precise relationship to the infinitesimal description in terms of the Lie pair $(\mathfrak{k}, \mathfrak{h})$ turns out to be subtle. Furthermore, as mentioned already in $[1,18]$, a physically desirable property of a kinematical spacetime is that orbits of the boost generators should be non-compact. To the best of our knowledge, a proof of this fact did not exist for many of the spacetimes in [18]. With this in mind, and based on a recent deformation-theoretic classification of kinematical Lie algebras [19-21], we revisited this problem and in [6] classified and showed the existence of simply-connected spatially isotropic homogeneous spacetimes in arbitrary dimension, making en passant a small correction to the $(3+1)$-dimensional classification in [18]. Another novel aspect of [6] was the classification of aristotelian spacetimes, which lack boost symmetry. One way to interpret this classification is as a generalisation of the classification of maximally symmetric rie-

\footnotetext{
${ }^{1}$ We refer to, e.g., [6] for further motivation and a (non-exhaustive) list of further references. While this work was under completion the interesting work [7] appeared which discusses similar aspects as this and our earlier work. Recently, also further interesting works, which fall in the realm of the kinematical Lie algebras and spacetimes, have appeared, see, e.g., [8-17].
} 
mannian and lorentzian spacetimes when we drop the requirement that there should exist an invariant metric.

Another way is to understand this work as a generalisation of the work of Bacry and Lévy-Leblond [1] when the assumption of parity and time reversal invariance and the restriction to $3+1$ dimensions is dropped. Simultaneously imposing parity and time reversal invariance ${ }^{2}$ selects the symmetric spaces, leading to the omission of some interesting spacetimes like, e.g., the non-reductive carrollian light cone LC and the torsional galilean spacetimes.

Let us emphasise that in identifying specific Lie algebra generators as "translations" or "boosts" one is actually implicitly referring to the homogeneous space. Indeed, the Lie algebra itself does not provide this interpretation. For example, by inspecting table 1 one recognises that the Minkowski (M) and AdS carrollian (AdSC) spacetimes share the same underlying Lie algebra. They are however different homogeneous spacetimes and the precise relationship between the kinematical Lie algebras and their spacetimes was also analysed in [6] and will be seen explicitly in the following analysis.

The methods employed in [6] are Lie algebraic and this means that in that paper we concentrated on geometrical properties which could be probed infinitesimally, such as determining the characteristic invariant structures (in low rank) that such a spacetime might possess, leaving the investigation of the orbits of the boosts to the present paper. Indeed, we will prove that the boosts do act with (generic) non-compact orbits in all spacetimes with the unsurprising exceptions of the aristotelian spacetimes (which have no boosts) and the riemannian symmetric spaces, where the "boosts" are actually rotations. ${ }^{3}$

To those ends we introduce exponential coordinates for each of the spacetimes in [6], relative to which we write down the fundamental vector fields which generate the action of the transitive Lie algebra. We also give explicit expressions for the invariant structure (lorentzian, galilean, carrollian, aristotelian) that the spacetime may possess. In addition, we determine the invariant connections which the homogeneous spacetimes admit (if any) and determine their torsion and curvature. We also pay particularly close attention to the orbits of the boost generators and in most cases show that the generic orbit is non-compact, as one would expect to be the case for any reasonable spacetime.

Finally, using modified exponential coordinates, we determine the infinitesimal (conformal) symmetries of the galilean and carrollian structures of our spacetimes. They are infinite-dimensional and reminiscent of BMS algebras. Many of the results already appear in $[3,29]$. Unobserved however was the close relation of the conformal symmetries of the (anti) de Sitter carrollian structure, belonging to null surfaces of (anti) de Sitter spacetime, and BMS symmetries. Section 10 can be read in large parts independently.

The paper is organised as follows. In section 2 we summarise the results of the classification in [6]. In tables 1 and 2 we list the simply-connected, spatially isotropic, homogeneous kinematical and aristotelian spacetimes, respectively. These are the spacetimes whose geometry we study in this paper. Figures 1, 2, and 3 summarise the relationships

\footnotetext{
${ }^{2}$ This operation is $\sigma(H)=-H$ and $\sigma(\boldsymbol{P})=-\boldsymbol{P}$ leaving the remaining generators unaltered.

${ }^{3}$ Since some of these spacetimes are well studied, there is necessarily some overlap with existing work, like the original works $[1,18]$ or more recent works that also discuss homogeneous spacetimes, e.g., [7, 22-28].
} 
between these spacetimes. These relationships take the form of limits which, in many cases, manifest themselves as contractions of the corresponding kinematical Lie algebras. Table 3 summarises some of the geometrical properties of the spacetimes in tables 1 and 2 . The list of spacetimes naturally breaks up into classes depending on which invariant structures (if any) the spacetimes possess: lorentzian, riemannian, galilean, carrollian and aristotelian. There are also exotic two-dimensional spacetimes with no discernible invariant structure. In section 3 we briefly review the basic notions of the local geometry of homogeneous spaces, tailored to the case at hand and compute the action of the rotations and boosts on the spacetimes. In section 4 we discuss the space of invariant connections for the reductive homogeneous spacetimes in tables 1 and 2 and calculate their torsion and curvature, paying particular attention to the existence of flat and/or torsion-free connections. In section 5 we discuss the lorentzian and riemannian homogeneous spaces and their limits. This leaves a few spacetimes which are not obviously obtained in this way and we discuss them separately: the torsional galilean homogeneous spacetimes are discussed in section 6 , the carrollian light cone in section 7 , the exotic two-dimensional spacetimes in section 8 , and the aristotelian spacetimes in section 9 . In section 10 we determine the infinitesimal (resp. conformal) symmetries of the galilean and carrollian spacetimes; namely, the vector fields which preserve (resp. rescale) the corresponding galilean and carrollian structure . The corresponding Lie algebras are typically infinite-dimensional and reminiscent of the BMS algebras. Finally, in section 11 we offer some conclusions. The paper contains two appendices: in appendix A we discuss the carrollian and galilean spacetimes in terms of modified exponential coordinates, which are the most convenient coordinates in order to discuss their symmetries, and in appendix B we record for convenience the Lie algebras of conformal Killing vectors on low-dimensional maximally symmetric riemannian manifolds.

\section{Homogeneous kinematical spacetimes}

We use the notation of [6], which we now review. Recall that a simply-connected homogeneous kinematical spacetime is described infinitesimally by a Lie pair $(\mathfrak{k}, \mathfrak{h})$. Here $\mathfrak{k}$ is a kinematical Lie algebra with $D$-dimensional space isotropy: namely, a real $\frac{(D+2)(D+1)}{2}$ dimensional Lie algebra with generators $J_{a b}, 1 \leq a<b \leq D$, spanning a Lie subalgebra isomorphic to $\mathfrak{s o}(D), B_{a}$ and $P_{a}$, for $1 \leq a \leq D$, transforming as vectors of $\mathfrak{s o}(D)$ and $H$ transforming as a scalar. The Lie subalgebra $\mathfrak{h}$ of $\mathfrak{k}$ contains $\mathfrak{s o}(D)$ and an $\mathfrak{s o}(D)$-vector representation, which is spanned by $\alpha B_{a}+\beta P_{a}, 1 \leq a \leq D$, for some non-zero $\alpha, \beta \in \mathbb{R}$. We choose a basis for $\mathfrak{k}$ such that $\mathfrak{h}$ is always spanned by $J_{a b}$ and $B_{a}$. In this fashion, the Lie brackets of $\mathfrak{k}$ uniquely specify the Lie pair $(\mathfrak{k}, \mathfrak{h})$.

Let us make a notational remark: we will refer to the generators $B_{a}$ as (infinitesimal) boosts, even though in some cases (e.g., the riemannian symmetric spaces) they act as rotations. A substantial part of the work that went into this paper was devoted to determining when the boosts really act like boosts and not, say, like rotations.

Notice that in writing down the Lie brackets of $\mathfrak{k}$, it is only necessary to list those brackets which do not involve $J_{a b}$ since those involving $J_{a b}$ are common for all kinematical Lie algebras and restate the fact that $J_{a b}$ span an $\mathfrak{s o}(D)$ subalgebra under which $B_{a}$ and 
$P_{a}$ are vectors and $H$ is a scalar. Explicitly, this reads

$$
\begin{aligned}
{\left[J_{a b}, J_{c d}\right] } & =\delta_{b c} J_{a d}-\delta_{a c} J_{b d}-\delta_{b d} J_{a c}+\delta_{a d} J_{b c} \\
{\left[J_{a b}, B_{c}\right] } & =\delta_{b c} B_{a}-\delta_{a c} B_{b} \\
{\left[J_{a b}, P_{c}\right] } & =\delta_{b c} P_{a}-\delta_{a c} P_{b} \\
{\left[J_{a b}, H\right] } & =0
\end{aligned}
$$

although we will use an abbreviated notation in which we do not write the $\mathfrak{s o}(D)$ indices explicitly. We write $\boldsymbol{J}, \boldsymbol{B}, \boldsymbol{P}$, and $H$ for the generators and rewrite the kinematical Lie brackets in (2.1) as

$$
[\boldsymbol{J}, \boldsymbol{J}]=\boldsymbol{J}, \quad[\boldsymbol{J}, \boldsymbol{B}]=\boldsymbol{B}, \quad[\boldsymbol{J}, \boldsymbol{P}]=\boldsymbol{P}, \quad \text { and } \quad[\boldsymbol{J}, H]=0 .
$$

For $D \neq 2$, any other brackets can be reconstructed unambiguously from the abbreviated expression since there is only one way to reintroduce indices in an $\mathfrak{s o}(D)$-equivariant fashion. For example,

$$
[H, \boldsymbol{B}]=\boldsymbol{P} \Longrightarrow\left[H, B_{a}\right]=P_{a} \quad \text { and } \quad[\boldsymbol{B}, \boldsymbol{P}]=H+\boldsymbol{J} \Longrightarrow\left[B_{a}, P_{b}\right]=\delta_{a b} H+J_{a b}
$$

In $D=3$ we may also have brackets of the form

$$
[\boldsymbol{P}, \boldsymbol{P}]=\boldsymbol{P} \Longrightarrow\left[P_{a}, P_{b}\right]=\epsilon_{a b c} P_{c} .
$$

Similarly, for $D=2, \epsilon_{a b}$ is rotationally invariant and can appear in Lie brackets. So we will write, e.g.,

$$
[H, \boldsymbol{B}]=\boldsymbol{B}+\widetilde{\boldsymbol{P}} \quad \text { for } \quad\left[H, B_{a}\right]=B_{a}+\epsilon_{a b} P_{b} .
$$

If the Lie subalgebra $\mathfrak{h}$ contains an ideal $\mathfrak{b}$ of $\mathfrak{k}$, we say that the Lie pair $(\mathfrak{k}, \mathfrak{h})$ is not effective. For a kinematical Lie algebra $\mathfrak{k}$, such an ideal is necessarily the one spanned by the boosts, which act trivially on the homogeneous spacetime. In such cases, we quotient by $\mathfrak{b}$ to arrive at an effective (by construction) Lie pair $(\mathfrak{a}, \mathfrak{r})$, where $\mathfrak{a}=\mathfrak{k} / \mathfrak{b}$ is an aristotelian Lie algebra and $\mathfrak{r} \cong \mathfrak{s o}(D)$ is the Lie subalgebra of rotations. The Lie pair $(\mathfrak{a}, \mathfrak{r})$ corresponds to an aristotelian spacetime. Not all aristotelian spacetimes arise in this way, and this justifies the separate classification of aristotelian Lie algebras and their corresponding spacetimes in [6, Appendix A].

\subsection{Classification}

We now summarise the results of [6]. Table 1 lists the (isomorphism classes of) simplyconnected, spatially isotropic, homogeneous spacetimes. We shall refer to them as "simplyconnected homogeneous kinematical spacetimes" from now on. These are described by Lie pairs $(\mathfrak{k}, \mathfrak{h})$, where $\mathfrak{k}$ is a kinematical Lie algebra with generators $\boldsymbol{J}, \boldsymbol{B}, \boldsymbol{P}, H$ and $\mathfrak{h}$ is the Lie subalgebra spanned by $\boldsymbol{J}, \boldsymbol{B}$. The first column is the label given in [6]. The second column specifies the value of $D$, where the dimension of the spacetime is $D+1$. The middle columns are the Lie brackets of $\mathfrak{k}$ in addition to the common kinematical Lie brackets in equation (2.2). It is tacitly assumed that when $D=1$, we set $\boldsymbol{J}=0$ whenever it appears. The final column contains any relevant comments, including the name of the spacetime if known. The table is divided by horizontal rules into five sections, from top to bottom: 
Lorentzian. These are the homogeneous kinematical spacetimes admitting an invariant lorentzian metric, which due to the dimension of the symmetry algebra must be maximally symmetric:

- Minkowski spacetime (M),

- de Sitter spacetime (dS), and

- anti de Sitter spacetime (AdS).

Riemannian. These are the homogeneous kinematical "spacetimes" admitting an invariant riemannian metric, which again must be maximally symmetric by dimension:

- euclidean space $(\mathbb{E})$,

- round sphere $(\mathbb{S})$, and

- hyperbolic space (H).

Galilean. These are the homogeneous kinematical spacetimes admitting an invariant galilean structure:

- galilean spacetime $(G)$,

- galilean de Sitter spacetime $\left(\mathrm{dSG}=\mathrm{dSG}_{-1}\right)$,

- torsional galilean de Sitter spacetime $\left(\mathrm{dSG}_{\gamma}, \gamma \in(-1,1]\right)$,

- galilean anti de Sitter spacetime $\left(A d S G=A d S G_{0}\right)$, and

- torsional galilean anti de Sitter spacetime $\left(\mathrm{AdSG}_{\chi}, \chi>0\right)$,

- a two-parameter family $\left(\mathrm{S} 12_{\gamma, \chi}\right)$ of three-dimensional galilean spacetimes interpolating between the torsional galilean (anti) de Sitter spacetimes.

Carrollian. These are the homogeneous kinematical spacetimes admitting an invariant carrollian structure:

- carrollian spacetime (C),

- carrollian de Sitter spacetime (dSC),

- carrollian anti de Sitter spacetime (AdSC), and

- carrollian light cone (LC).

These spacetimes are identifiable as null hypersurfaces in homogeneous, lorentzian kinematical spacetimes in one dimension higher: $M$ for $C$ and LC, AdS for AdSC and dS for dSC. In particular, the image of the embedding $L C \subset M$ is the future light cone. ${ }^{4}$

Exotic. These are two-dimensional kinematical spacetimes without any discernible invariant structures.

\footnotetext{
${ }^{4}$ Strictly speaking, it is the future light cone if $D>1$ and its universal cover if $D=1$, a fact that was initially glossed over in [6].
} 


\begin{tabular}{|c|c|c|c|c|c|c|c|}
\hline Label & $D$ & & Non-zero Lie brackets in addition to & {$[\boldsymbol{J}, \boldsymbol{J}]=\boldsymbol{J},[\boldsymbol{J}$} & $\boldsymbol{B}]=\boldsymbol{B},[\boldsymbol{J}, \boldsymbol{P}]=\boldsymbol{P}$ & & Comments \\
\hline S1 & $\geq 1$ & {$[H, \boldsymbol{B}]=-\boldsymbol{P}$} & & {$[\boldsymbol{B}, \boldsymbol{B}]=\boldsymbol{J}$} & {$[\boldsymbol{B}, \boldsymbol{P}]=H$} & & M \\
\hline S2 & $\geq 2$ & {$[H, \boldsymbol{B}]=-\boldsymbol{P}$} & {$[H, \boldsymbol{P}]=-\boldsymbol{B}$} & {$[\boldsymbol{B}, \boldsymbol{B}]=\boldsymbol{J}$} & {$[\boldsymbol{B}, \boldsymbol{P}]=H$} & {$[\boldsymbol{P}, \boldsymbol{P}]=-\boldsymbol{J}$} & dS \\
\hline S3 & $\geq 1$ & {$[H, \boldsymbol{B}]=-\boldsymbol{P}$} & {$[H, \boldsymbol{P}]=\boldsymbol{B}$} & {$[\boldsymbol{B}, \boldsymbol{B}]=\boldsymbol{J}$} & {$[\boldsymbol{B}, \boldsymbol{P}]=H$} & {$[P, P]=J$} & AdS \\
\hline S4 & $\geq 1$ & {$[H, \boldsymbol{B}]=\boldsymbol{P}$} & & {$[\boldsymbol{B}, \boldsymbol{B}]=-\boldsymbol{J}$} & {$[\boldsymbol{B}, \boldsymbol{P}]=H$} & & $\mathbb{E}$ \\
\hline S5 & $\geq 1$ & {$[H, \boldsymbol{B}]=\boldsymbol{P}$} & {$[H, \boldsymbol{P}]=-\boldsymbol{B}$} & {$[\boldsymbol{B}, \boldsymbol{B}]=-\boldsymbol{J}$} & {$[\boldsymbol{B}, \boldsymbol{P}]=H$} & {$[\boldsymbol{P}, \boldsymbol{P}]=-\boldsymbol{J}$} & S \\
\hline S6 & $\geq 1$ & {$[H, \boldsymbol{B}]=\boldsymbol{P}$} & {$[H, \boldsymbol{P}]=\boldsymbol{B}$} & {$[\boldsymbol{B}, \boldsymbol{B}]=-\boldsymbol{J}$} & {$[\boldsymbol{B}, \boldsymbol{P}]=H$} & {$[P, P]=J$} & H \\
\hline S7 & $\geq 1$ & {$[H, \boldsymbol{B}]=-\boldsymbol{P}$} & & & & & G \\
\hline S8 & $\geq 1$ & {$[H, \boldsymbol{B}]=-\boldsymbol{P}$} & {$[H, \boldsymbol{P}]=-\boldsymbol{B}$} & & & & $\mathrm{dSG}=\mathrm{dSG}_{\gamma=-1}$ \\
\hline $\mathrm{S} 9_{\gamma}$ & $\geq 1$ & {$[H, \boldsymbol{B}]=-\boldsymbol{P}$} & {$[H, \boldsymbol{P}]=\gamma \boldsymbol{B}+(1+\gamma) \boldsymbol{P}$} & & & & $\mathrm{dSG}_{\gamma \in(-1,1]}$ \\
\hline S10 & $\geq 1$ & {$[H, \boldsymbol{B}]=-\boldsymbol{P}$} & {$[H, P]=\boldsymbol{B}$} & & & & $A d S G=\operatorname{AdSG}_{\chi=0}$ \\
\hline $\mathrm{S} 11_{\chi}$ & $\geq 1$ & {$[H, \boldsymbol{B}]=-\boldsymbol{P}$} & {$[H, \boldsymbol{P}]=\left(1+\chi^{2}\right) \boldsymbol{B}+2 \chi \boldsymbol{P}$} & & & & $\operatorname{AdSG}_{\chi>0}$ \\
\hline $\mathrm{S} 12_{\gamma, \chi}$ & 2 & {$[H, \boldsymbol{B}]=-\boldsymbol{P}$} & {$[H, \boldsymbol{P}]=(1+\gamma) \boldsymbol{P}-\chi \widetilde{\boldsymbol{P}}+\gamma \boldsymbol{B}-\chi \widetilde{\boldsymbol{B}}$} & & & & $\gamma \in[-1,1), \chi>0$ \\
\hline $\mathrm{S} 13$ & $\geq 2$ & & & & {$[\boldsymbol{B}, \boldsymbol{P}]=H$} & & $\mathrm{C}$ \\
\hline S14 & $\geq 2$ & & {$[H, \boldsymbol{P}]=-\boldsymbol{B}$} & & {$[\boldsymbol{B}, \boldsymbol{P}]=H$} & {$[\boldsymbol{P}, \boldsymbol{P}]=-\boldsymbol{J}$} & dSC \\
\hline S15 & $\geq 2$ & & {$[H, \boldsymbol{P}]=\boldsymbol{B}$} & & {$[\boldsymbol{B}, \boldsymbol{P}]=H$} & {$[P, P]=J$} & AdSC \\
\hline S16 & $\geq 1$ & {$[H, B]=\boldsymbol{B}$} & {$[H, \boldsymbol{P}]=-\boldsymbol{P}$} & & {$[\boldsymbol{B}, \boldsymbol{P}]=H+\boldsymbol{J}$} & & LC \\
\hline S17 & 1 & {$[H, B]=-P$} & & & {$[B, P]=-H-2 P$} & & \\
\hline S18 & 1 & {$[H, B]=H$} & & & {$[B, P]=-P$} & & \\
\hline $\mathrm{S} 19_{\chi}$ & 1 & {$[H, B]=(1+\chi) H$} & & & {$[B, P]=(1-\chi) P$} & & $\chi>0$ \\
\hline $\mathrm{S} 20_{\chi}$ & 1 & {$[H, B]=-P$} & & & {$[B, P]=-\left(1+\chi^{2}\right) H-2 \chi P$} & & $\chi>0$ \\
\hline
\end{tabular}

Table 1. Simply-connected homogeneous $(D+1)$-dimensional kinematical spacetimes. The horizontal rules separate the lorentzian, riemannian, galilean, carrollian and exotic spacetimes. For further properties see table 3 .

Since, in two dimensions, it is largely a matter of convention what one calls space and time, ${ }^{5}$ some of the spacetimes become accidentally pairwise isomorphic when $D=1$ : namely, $C \cong G, d S \cong A d S, d S C \cong A d S G$ and $A d S C \cong d S G$. In order to arrive at a one-to-one correspondence between the rows of the table and the isomorphism class of simply-connected homogeneous spacetimes, we write $D \geq 2$ for dS, C, dSC and AdSC.

Table 2 lists the isomorphism classes of simply-connected aristotelian spacetimes. Homogeneous aristotelian spacetimes are always reductive, and they admit simultaneously invariant galilean and carrollian structures. We label them as A\# as opposed to S\#, for mnemonic reasons:

- A21 is the static aristotelian spacetime (S),

- A22 is the torsional static aristotelian spacetime (TS),

- $\mathrm{A} 23_{\varepsilon}$ are the Einstein static spacetime $\mathbb{R} \times \mathbb{S}^{D}$ for $\varepsilon=+1$ and the hyperbolic version $\mathbb{R} \times \mathbb{H}^{D}$ for $\varepsilon=-1$, and

- A24 is a three-dimensional static spacetime with underlying manifold the Heisenberg Lie group.

\subsection{Geometric limits}

Many of the above spacetimes are connected by geometric limits, some of which manifest themselves as contractions of the kinematical Lie algebras. Figure 1 illustrates these limits for generic $D \geq 3$. For $D \leq 2$, the picture is modified in a way that will be explained below.

\footnotetext{
${ }^{5}$ While true when discussing the geometry of homogeneous spacetimes, there is of course a physical distinction between space and time: time translations are generated by the hamiltonian, whose spectrum one often requires to be bounded from below, whereas the spectrum of spatial translations is not subject to such a requirement.
} 


\begin{tabular}{|l|c|r|l|}
\hline Label & $D$ & Non-zero Lie brackets in addition to $[\boldsymbol{J}, \boldsymbol{J}]=\boldsymbol{J}$ and $[\boldsymbol{J}, \boldsymbol{P}]=\boldsymbol{P}$ & Comments \\
\hline A21 & $\geq 0$ & & $\mathrm{~S}$ \\
A22 & $\geq 1$ & {$[H, \boldsymbol{P}]=\boldsymbol{P}$} & TS \\
A23 & $\geq 2$ & {$[\boldsymbol{P}, \boldsymbol{P}]=-\boldsymbol{J}$} & $\mathbb{R} \times \mathbb{S}^{D}$ \\
A23 & $\geq 2$ & {$[\boldsymbol{P}, \boldsymbol{P}]=\boldsymbol{J}$} & $\mathbb{R} \times \mathbb{H}^{D}$ \\
A24 & 2 & {$[\boldsymbol{P}, \boldsymbol{P}]=H$} & \\
\hline
\end{tabular}

Table 2. Simply-connected homogeneous $(D+1)$-dimensional aristotelian spacetimes. For further properties see table 3 .

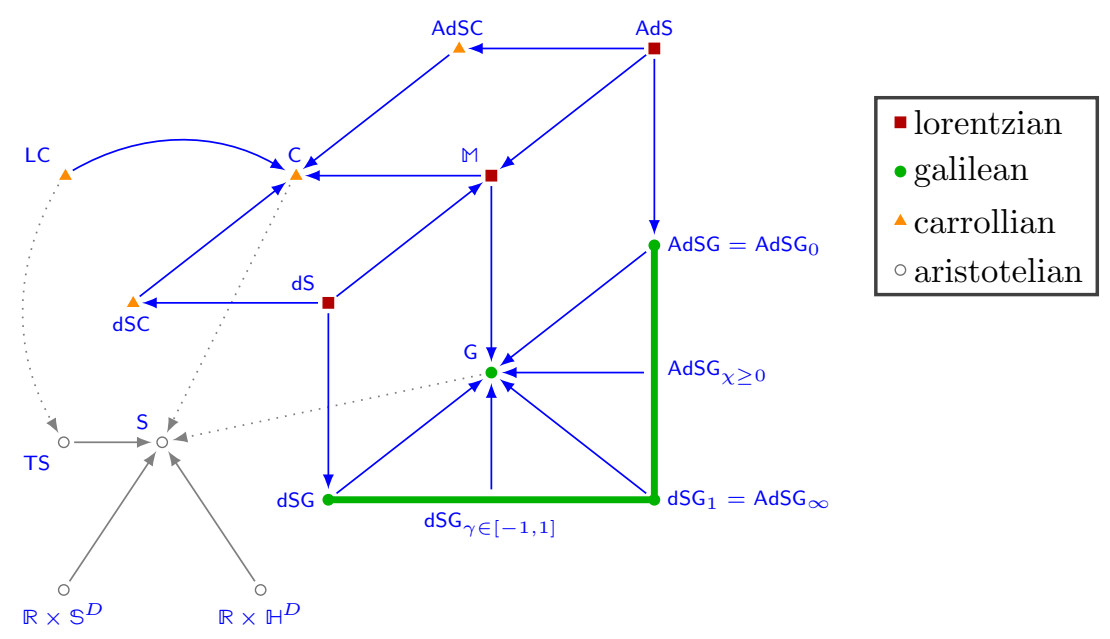

Figure 1. Homogeneous spacetimes in dimension $D+1 \geq 4$ and their limits.

There are several types of limits displayed in figure 1:

- flat limits in which the curvature of the canonical connection goes to zero: AdS $\rightarrow$ M, $\mathrm{dS} \rightarrow \mathrm{M}, \mathrm{AdSC} \rightarrow \mathrm{C}, \mathrm{dSC} \rightarrow \mathrm{C}, \mathrm{AdSG} \rightarrow \mathrm{G}$ and $\mathrm{dSG} \rightarrow \mathrm{G}$

- non-relativistic limits in which the speed of light goes to infinity (morally speaking): $\mathrm{M} \rightarrow \mathrm{G}, \mathrm{AdS} \rightarrow$ AdSG and dS $\rightarrow \mathrm{dSG}$

in this limit there is still the notion of relativity, it just differs from the standard lorentzian one. Therefore, although it might be more appropriate to call it the "galilean limit", we will conform to the literature and call it the non-relativistic limit.

- ultra-relativistic limits in which the speed of light goes to zero (again, morally speaking) $\mathrm{M} \rightarrow \mathrm{C}, \mathrm{AdS} \rightarrow$ AdSC and $\mathrm{dS} \rightarrow \mathrm{dSC}$.

- limits to non-effective Lie pairs which, after quotienting by the ideal generated by the boosts, result in an aristotelian spacetime: the dotted arrows LC $\rightarrow$ TS, C $\rightarrow$ S and $\mathrm{G} \rightarrow \mathrm{S}$;

- $\mathrm{LC} \rightarrow \mathrm{C}$, which is a contraction of $\mathfrak{s o}(D+1,1)$;

- $\mathrm{dSG}_{\gamma} \rightarrow \mathrm{G}$ and $\mathrm{AdSG}_{\gamma} \rightarrow \mathrm{G}$, which are contractions of the corresponding kinematical Lie algebras; 


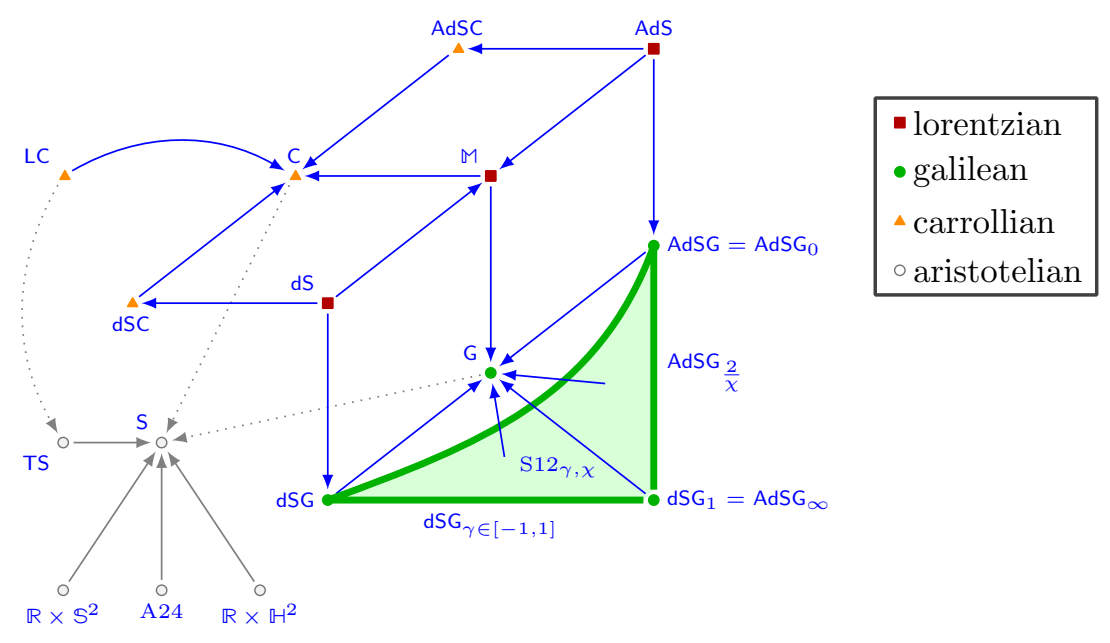

Figure 2. Three-dimensional homogeneous spacetimes and their limits.

- limits between aristotelian spacetimes $\mathrm{TS} \rightarrow \mathrm{S}, \mathbb{R} \times \mathbb{S}^{D} \rightarrow \mathrm{S}$ and $\mathbb{R} \times \mathbb{H}^{D} \rightarrow \mathrm{S}$; and

- a limit $\lim _{\chi \rightarrow \infty} \mathrm{AdSG}_{\chi}=\mathrm{dSG}$, which is not due to a contraction of the kinematical Lie algebras.

We can compose these limits like arrows in a commutative diagram, and therefore we do not show all the possible limits. All these limits are explained in [6].

The situation in $D \leq 2$ is slightly different. As can be seen in tables 1 and 2 , there are two classes of spacetimes which are unique to $D=2$ : a two-parameter family of galilean spacetimes $\left(\mathrm{S} 12_{\gamma, \chi}\right.$, for $\gamma \in[-1,1)$ and $\left.\chi>0\right)$ and the aristotelian spacetime A24. We can understand this latter spacetime as the group manifold of the three-dimensional Heisenberg group. The former two-parameter family interpolates between the torsional galilean (anti) de Sitter spacetimes. As shown in figure 2, the limit $\gamma \rightarrow 1$ of $\mathrm{S}_{2} 2_{\gamma, \chi}$ is $\mathrm{AdSG}_{2 / \chi}$, so that if we then take $\chi \rightarrow 0$, we arrive at $\mathrm{dSG}_{1}$. More generally, the limit $\chi \rightarrow 0$ of $\mathrm{S}_{2} 2_{\gamma, \chi}$ is $\mathrm{dSG}_{\gamma}$, whereas the limit $\chi \rightarrow \infty$ is independent of $\gamma$ and given by AdSG.

Table 1 shows that there are four classes of two-dimensional spacetimes unique to $D=1$. These spacetimes are affine but have no discernible structure. In [6] we describe a number of limits involving these two-dimensional spacetimes. Figure 3 illustrates the relationship between the two-dimensional spacetimes. This figure includes the riemannian maximally symmetric spaces which are missing from figures 1 and 2 .

\subsection{Geometrical properties}

In table 3 we summarise the basic properties of the homogeneous kinematical spacetimes in table 1 and aristotelian spacetimes in table 2. The first column is our label in this paper, the second column specifies the value of $D$, where the dimension of the spacetime is $D+1$. The columns labelled "R", "S", and "A" indicate whether or not the spacetime is reductive, symmetric, or affine, respectively. A $\checkmark$ indicates that it is. A $(\checkmark)$ in the affine column reflects the existence of an invariant connection (other than the canonical connection) with vanishing torsion and curvature. The columns labelled "L", "E", "G", and "C" indicate the 


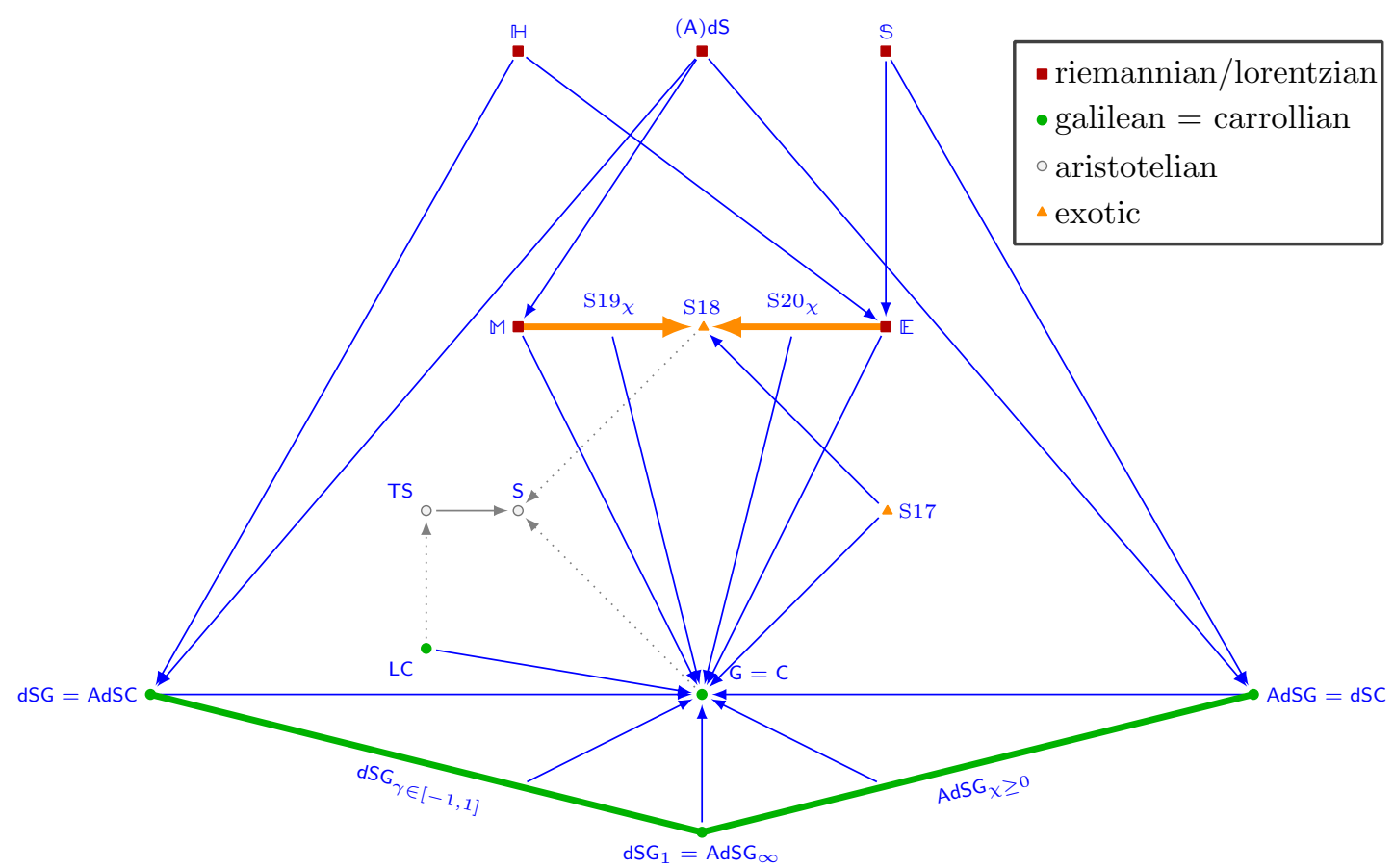

Figure 3. Two-dimensional homogeneous spacetimes and their limits.

kind of invariant structures the spacetime possesses: lorentzian, riemannian ("euclidean"), galilean, and carrollian, respectively. Again a $\checkmark$ indicates that the spacetime possesses that structure. The columns "P", "T", and "PT" indicate whether the spacetime is invariant under parity, time reversal or their combination, respectively, with $\checkmark$ signalling that they do. The column "B" summarises results of the current paper (to be found below) and indicates whether the boosts act with non-compact orbits in a kinematical spacetime. The columns " $\Theta$ " and " $\Omega$ " tell us, respectively, about the torsion and curvature of the canonical invariant connection for the reductive spacetimes (that is, all but LC). A " $\neq 0$ " indicates the presence of torsion, curvature, or both torsion and curvature. Its absence indicates that the connection is torsion-free, flat, or both. The final column contains any relevant comments, including, when known, the name of the spacetime.

The table is divided into six sections. The first four correspond to lorentzian, euclidean, galilean and carrollian spacetimes. The fifth section contains two-dimensional spacetimes with no invariant structure of these kinds. The sixth and last section contains the aristotelian spacetimes. Some of the spacetimes which exist for all $D \geq 1$ become accidentally pairwise isomorphic in $D=1$ : namely, $\mathrm{C} \cong \mathrm{G}, \mathrm{dS} \cong \mathrm{AdS}, \mathrm{dSC} \cong \mathrm{AdSG}$ and $\mathrm{AdSC} \cong \mathrm{dSG}$. These accidental isomorphisms explain why we write $D \geq 2$ for carrollian, de Sitter, and carrollian (anti) de Sitter. In this way no two rows are isomorphic, and hence every row in the table specifies a unique simply-connected homogeneous spacetime, up to isomorphism. 


\begin{tabular}{|c|c|c|c|c|c|c|c|c|c|c|c|c|c|c|c|}
\hline Label & $D$ & $R$ & $S$ & $A$ & $L$ & $E$ & $G$ & $C$ & $P$ & $T$ & $P T$ & $B$ & $\Theta$ & $\Omega$ & Comments \\
\hline S1 & $\geq 1$ & $\checkmark$ & $\checkmark$ & $\checkmark$ & $\checkmark$ & & & & $\checkmark$ & $\checkmark$ & $\checkmark$ & $\checkmark$ & & & $M$ \\
\hline S2 & $\geq 2$ & $\checkmark$ & $\checkmark$ & & $\checkmark$ & & & & $\checkmark$ & $\checkmark$ & $\checkmark$ & $\checkmark$ & & $\neq 0$ & dS \\
\hline S3 & $\geq 1$ & $\checkmark$ & $\checkmark$ & & $\checkmark$ & & & & $\checkmark$ & $\checkmark$ & $\checkmark$ & $\checkmark$ & & $\neq 0$ & AdS \\
\hline S4 & $\geq 1$ & $\checkmark$ & $\checkmark$ & $\checkmark$ & & $\checkmark$ & & & $\checkmark$ & $\checkmark$ & $\checkmark$ & & & & $\mathbb{E}$ \\
\hline S5 & $\geq 1$ & $\checkmark$ & $\checkmark$ & & & $\checkmark$ & & & $\checkmark$ & $\checkmark$ & $\checkmark$ & & & $\neq 0$ & $\mathbb{S}$ \\
\hline $\mathrm{S} 6$ & $\geq 1$ & $\checkmark$ & $\checkmark$ & & & $\checkmark$ & & & $\checkmark$ & $\checkmark$ & $\checkmark$ & & & $\neq 0$ & $H$ \\
\hline S7 & $\geq 1$ & $\checkmark$ & $\checkmark$ & $\checkmark$ & & & $\checkmark$ & & $\checkmark$ & $\bar{\checkmark}$ & $\checkmark$ & $\checkmark$ & & & $G$ \\
\hline S8 & $\geq 1$ & $\checkmark$ & $\checkmark$ & $(\checkmark)$ & & & $\checkmark$ & & $\checkmark$ & $\checkmark$ & $\checkmark$ & $\checkmark$ & & $\neq 0$ & dSG \\
\hline $\mathrm{S} 9_{\gamma \neq 0}$ & $\geq 1$ & $\checkmark$ & & $(\checkmark)$ & & & $\checkmark$ & & $\checkmark$ & & & $\checkmark$ & $\neq 0$ & $\neq 0$ & $\mathrm{dSG}_{\gamma}, 0 \neq \gamma \in(-1,1]$ \\
\hline $\mathrm{S} 9_{0}$ & $\geq 1$ & $\checkmark$ & & $(\checkmark)$ & & & $\checkmark$ & & $\checkmark$ & & & $\checkmark$ & $\neq 0$ & & $\mathrm{dSG}_{0}$ \\
\hline S10 & $\geq 1$ & $\checkmark$ & $\checkmark$ & & & & $\checkmark$ & & $\checkmark$ & $\checkmark$ & $\checkmark$ & $\checkmark$ & & $\neq 0$ & AdSG \\
\hline $\mathrm{S} 11_{\chi}$ & $\geq 1$ & $\checkmark$ & & & & & $\checkmark$ & & $\checkmark$ & & & $\checkmark$ & $\neq 0$ & $\neq 0$ & $\operatorname{AdSG}_{\chi}, \chi>0$ \\
\hline $\mathrm{S} 12_{\gamma, \chi}$ & 2 & $\checkmark$ & & & & & $\checkmark$ & & $\checkmark$ & & & $\checkmark$ & $\neq 0$ & $\neq 0$ & $\gamma \in[-1,1), \chi>0$ \\
\hline S13 & $\geq 2$ & $\checkmark$ & $\checkmark$ & $\checkmark$ & & & & $\checkmark$ & $\checkmark$ & $\checkmark$ & $\checkmark$ & $\checkmark$ & & & $\mathrm{C}$ \\
\hline S14 & $\geq 2$ & $\checkmark$ & $\checkmark$ & & & & & $\checkmark$ & $\checkmark$ & $\checkmark$ & $\checkmark$ & $\checkmark$ & & $\neq 0$ & $\mathrm{dSC}$ \\
\hline S15 & $\geq 2$ & $\checkmark$ & $\checkmark$ & & & & & $\checkmark$ & $\checkmark$ & $\checkmark$ & $\checkmark$ & $\checkmark$ & & $\neq 0$ & AdSC \\
\hline S16 & $\geq 1$ & & & $\checkmark)_{D=1}$ & & & & $\checkmark$ & $\checkmark$ & & & $\checkmark$ & & & LC \\
\hline S17 & 1 & $\checkmark$ & $\checkmark$ & $\checkmark$ & & & & & & & $\checkmark$ & $\checkmark$ & & & \\
\hline S18 & 1 & $\checkmark$ & $\checkmark$ & $\checkmark$ & & & & & & $\checkmark$ & & $\checkmark$ & & & \\
\hline $\mathrm{S} 19_{\chi}$ & 1 & $\checkmark$ & $\checkmark$ & $\checkmark$ & & & & & & $\checkmark$ & & $\checkmark$ & & & $\chi>0$ \\
\hline $\mathrm{S} 20_{\chi}$ & 1 & $\checkmark$ & $\checkmark$ & $\checkmark$ & & & & & & & $\checkmark$ & $\checkmark$ & & & $\chi>0$ \\
\hline A21 & $\geq 0$ & $\checkmark$ & $\checkmark$ & $\checkmark$ & $\checkmark$ & $\checkmark$ & $\checkmark$ & $\checkmark$ & $\checkmark$ & $\checkmark$ & $\checkmark$ & & & & $\mathrm{S}$ \\
\hline $\mathrm{A} 22$ & $\geq 1$ & $\checkmark$ & & $(\checkmark)$ & $\checkmark$ & $\checkmark$ & $\checkmark$ & $\checkmark$ & $\checkmark$ & & & & $\neq 0$ & & TS \\
\hline $\mathrm{A} 23_{+1}$ & $\geq 2$ & $\checkmark$ & $\checkmark$ & & $\checkmark$ & $\checkmark$ & $\checkmark$ & $\checkmark$ & $\checkmark$ & $\checkmark$ & $\checkmark$ & & & $\neq 0$ & $\mathbb{R} \times \mathbb{S}^{D}$ \\
\hline $\mathrm{A} 23_{-1}$ & $\geq 2$ & $\checkmark$ & $\checkmark$ & & $\checkmark$ & $\checkmark$ & $\checkmark$ & $\checkmark$ & $\checkmark$ & $\checkmark$ & $\checkmark$ & & & $\neq 0$ & $\mathbb{R} \times \mathbb{H}^{D}$ \\
\hline A24 & 2 & $\checkmark$ & & $(\checkmark)$ & $\checkmark$ & $\checkmark$ & $\checkmark$ & $\checkmark$ & $\checkmark$ & & & & $\neq 0$ & & \\
\hline
\end{tabular}

Table 3. Properties of simply-connected homogeneous spacetimes. This table describes if a $D+1$ dimensional kinematical spacetime (table 1) or aristotelian spacetime (table 2) is reductive $(\mathrm{R})$, symmetric $(\mathrm{S})$ or affine $(\mathrm{A})$. A spacetime might exhibit a lorentzian $(\mathrm{L})$, riemannian $(\mathrm{E})$, galilean $(\mathrm{G})$ or carrollian $(\mathrm{C})$ structure, and be invariant under parity $(\mathrm{P})$, time reversal $(\mathrm{T})$ or their combination $(\mathrm{PT})$. The boosts (B) may act with non-compact orbits. Furthermore the canonical connection of a reductive spacetime might be have torsion $(\Theta)$ and/or curvature $(\Omega)$.

\section{Local geometry of homogeneous spacetimes}

In this section, we review some basic properties of homogeneous spaces, tailored to the cases of interest. We discuss exponential coordinates, the fundamental vector fields, the group action, the action of rotations and boosts, the soldering form, and the vielbein. In addition, we discuss the invariant connections on a reductive homogeneous space.

\subsection{Exponential coordinates}

Let $M=\mathcal{K} / \mathcal{H}$ be a kinematical spacetime with associated Lie pair $(\mathfrak{k}, \mathfrak{h})$ in which $\mathfrak{k}$ is a kinematical Lie algebra and $\mathfrak{h}$ is the Lie subalgebra spanned by the rotations $J_{a b}$ and the boosts $B_{a}$. The identification of $M$ with the coset manifold $\mathcal{K} / \mathcal{H}$ singles out a point $o \in M$ corresponding to the identity coset. We call it the origin of $M$. Any other point in $M$ would be equally valid as an "origin", but that choice would induce an identification 
with a different coset manifold since the new origin typically has a different, but of course conjugate, stabiliser subgroup.

The action of $\mathcal{K}$ on $M$ is induced by left multiplication on $\mathcal{K}$. If we let $\varpi: \mathcal{K} \rightarrow M=$ $\mathcal{K} / \mathcal{H}$ denote the canonical surjection, then for all $g \in \mathcal{K}$, we have that

$$
g \cdot \varpi(k)=\varpi(g k) .
$$

This is well defined because if $\varpi(k)=\varpi\left(k^{\prime}\right)$, then there is some $h \in \mathcal{H}$ such that $k^{\prime}=k h$ and by associativity of the group multiplication $g k^{\prime}=g(k h)=(g k) h$, so that $\varpi(g k)=\varpi\left(g k^{\prime}\right)$.

Now consider acting with $g \in \mathcal{K}$ on the origin. If $g \in \mathcal{H}, g \cdot o=o$, so this suggests the following. Let $\mathfrak{m}=\operatorname{span}\left\{P_{a}, H\right\}$ denote a vector space complement of $\mathfrak{h}$ in $\mathfrak{k}$ and define $\exp _{o}: \mathfrak{m} \rightarrow M$ by

$$
\exp _{o}(X)=\exp (X) \cdot o \quad \text { for all } X \in \mathfrak{m} .
$$

This map defines a local diffeomorphism from a neighbourhood of 0 in $\mathfrak{m}$ and a neighbourhood of $o$ in $M$, and hence it defines exponential coordinates near $o$ via $\sigma: \mathbb{R}^{D+1} \rightarrow M$, where $\sigma(t, \boldsymbol{x})=\exp _{o}(t H+\boldsymbol{x} \cdot \boldsymbol{P})$. This coordinate chart has an origin $o \in M$, which is the point with coordinates $(t, \boldsymbol{x})=(0, \mathbf{0})$. We may translate this coordinate chart from the origin to any other point of $M$ via the action of the group and in this way arrive at an exponential coordinate atlas for $M$. It is not the only natural coordinate system associated with a choice of basis for $\mathfrak{m}$. Indeed, it is often more convenient computationally to use modified exponential coordinates via products of exponentials, say, $\sigma^{\prime}(t, \boldsymbol{x})=\exp (t H) \exp (\boldsymbol{x} \cdot \boldsymbol{P}) \cdot o$. For most of this work we have opted to use strict exponential coordinates in our calculations for uniformity and to ease comparison: the exception being the determination of the symmetries, where modified exponential coordinates (as described in appendix A) allow for a more uniform description.

There are some natural questions one can ask about the local diffeomorphism $\exp _{o}$ : $\mathfrak{m} \rightarrow M$ or, equivalently, the local diffeomorphism $\sigma: \mathbb{R}^{D+1} \rightarrow M$. One can ask how much of $M$ is covered by the image of $\exp _{o}$. We say that $M$ is exponential if $M=\exp _{o}(\mathfrak{m})$ and weakly exponential if $M=\overline{\exp _{o}(\mathfrak{m})}$, where the bar denotes topological closure. Similarly, we can ask about the domain of validity of exponential coordinates: namely, the subspace of $\mathbb{R}^{D+1}$ where $\sigma$ remains injective. In particular, if $\sigma$ is everywhere injective, does it follow that $\sigma$ is also surjective? We know very little about these questions for general homogeneous spaces, even in the reductive case. However, there are some general theorems for the case of $M$ a symmetric space.

Theorem 1 (Voglaire [30]). Let $M=\mathcal{K} / \mathcal{H}$ be a connected symmetric space with symmetric decomposition $\mathfrak{k}=\mathfrak{h} \oplus \mathfrak{m}$ and define $\exp _{o}: \mathfrak{m} \rightarrow M$. Then the following are equivalent:

1. $\exp _{o}: \mathfrak{m} \rightarrow M$ is injective

2. $\exp _{o}: \mathfrak{m} \rightarrow M$ is a global diffeomorphism

3. $M$ is simply connected and for no $X \in \mathfrak{m}$, does $\operatorname{ad}_{X}: \mathfrak{k} \rightarrow \mathfrak{k}$ have purely imaginary eigenvalues. 
Since our homogeneous spaces are by assumption simply-connected, the last criterion in the theorem is infinitesimal and, therefore, easily checked from the Lie algebra. This result makes it a relatively simple task to inspect table 1 and determine for which of the symmetric spaces the last criterion holds by studying the eigenvalues of $\operatorname{ad}_{H} \operatorname{and}_{\operatorname{ad}_{P_{a}}}$ on $\mathfrak{k}$. Inspection of table 1 shows that $M, \mathbb{E}, \mathbb{H}, \mathrm{G}, \mathrm{dSG}, \mathrm{C}$ and AdSC satisfy criterion (3) above and hence that the exponential coordinates define a diffeomorphism to $\mathbb{R}^{D+1}$ for these spaces. It also follows by inspection that dS, AdS, $\mathbb{S}$, AdSG and dSC do not satisfy criterion (3) above and hence the exponential coordinates do not give us a global chart. We will be able to confirm this directly when we calculate the soldering form for these symmetric spaces.

Concerning the (weak) exponentiality of symmetric spaces, we will make use of the following result.

Theorem 2 (Rozanov [31]). Let $M=\mathcal{K} / \mathcal{H}$ be a symmetric space with $\mathcal{K}$ connected. Then

1. If $\mathcal{K}$ is solvable, then $M$ is weakly exponential.

2. $M$ is weakly exponential if and only if $\widehat{M}=\widehat{\mathcal{K}} / \widehat{\mathcal{H}}$ is weakly exponential, where $\widehat{\mathcal{K}}=$ $\mathcal{K} / \operatorname{Rad}(\mathcal{K})$ and similarly for $\widehat{\mathcal{H}}$, where the radical $\operatorname{Rad}(\mathcal{K})$ is the maximal connected solvable normal subgroup of $\mathcal{K}$.

The Lie algebra of $\operatorname{Rad}(\mathcal{K})$ is the radical of the Lie algebra $\mathfrak{k}$, which is the maximal solvable ideal, and can be calculated efficiently via the identification $\operatorname{rad} \mathfrak{k}=[\mathfrak{k}, \mathfrak{k}]^{\perp}$, namely, the radical is the perpendicular subspace (relative to the Killing form, which may be degenerate) of the first derived ideal.

It will follow from Theorem 2 that AdSG is weakly exponential.

\subsection{The group action and the fundamental vector fields}

The action of the group $\mathcal{K}$ on $M$ is induced by left multiplication on the group. Indeed, we have a commuting square

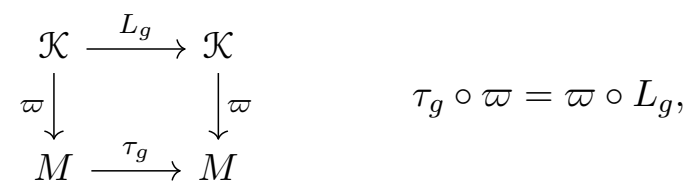

where $L_{g}$ is the diffeomorphism of $\mathcal{K}$ given by left multiplication by $g \in \mathcal{K}$ and $\tau_{g}$ is the diffeomorphism of $M$ given by acting with $g$. In terms of exponential coordinates, we have $g \cdot(t, \boldsymbol{x})=\left(t^{\prime}, \boldsymbol{x}^{\prime}\right)$ where

$$
g \exp (t H+\boldsymbol{x} \cdot \boldsymbol{P})=\exp \left(t^{\prime} H+\boldsymbol{x}^{\prime} \cdot \boldsymbol{P}\right) h
$$

for some $h \in \mathcal{H}$ which typically depends on $g, t$, and $\boldsymbol{x}$.

If $g=\exp (X)$ with $X \in \mathfrak{h}$ and if $A=t H+\boldsymbol{x} \cdot \boldsymbol{P} \in \mathfrak{m}$, the following identity will be useful:

$$
\exp (X) \exp (A)=\exp \left(\exp \left(\operatorname{ad}_{X}\right) A\right) \exp (X)
$$


If $M$ is reductive, so that $[\mathfrak{h}, \mathfrak{m}] \subset \mathfrak{m}$, which is the case for all but one of the kinematical spacetimes, then $\operatorname{ad}_{X} A \in \mathfrak{m}$ and, since $\mathfrak{m}$ is a finite-dimensional vector space and hence topologically complete, $\exp \left(\operatorname{ad}_{X}\right) A \in \mathfrak{m}$ as well. In this case, we may act on the origin $o \in M$, which is stabilised by $\mathcal{H}$, to rewrite equation (3.5) as

$$
\exp (X) \exp _{o}(A)=\exp _{o}\left(\exp \left(\operatorname{ad}_{X}\right) A\right)
$$

or, in terms of $\sigma$,

$$
\exp (X) \sigma(t, \boldsymbol{x})=\sigma\left(\exp \left(\operatorname{ad}_{X}\right)(t H+\boldsymbol{x} \cdot \boldsymbol{P})\right)=\sigma\left(t^{\prime}, \boldsymbol{x}^{\prime}\right) .
$$

This latter way of writing the equation shows the action of $\exp (X)$ on the exponential coordinates $(t, \boldsymbol{x})$, namely

$$
(t, \boldsymbol{x}) \mapsto\left(t^{\prime}, \boldsymbol{x}^{\prime}\right) \quad \text { where } \quad t^{\prime} H+\boldsymbol{x}^{\prime} \cdot \boldsymbol{P}:=\exp \left(\operatorname{ad}_{X}\right)(t H+\boldsymbol{x} \cdot \boldsymbol{P}) .
$$

As we will show below, the rotations act in the usual way: they leave $t$ invariant and rotate $\boldsymbol{x}$, so we will normally concentrate on the action of the boosts and translations. This requires calculating, for example,

$$
\exp \left(v^{a} P_{a}\right) \sigma(t, \boldsymbol{x})=\sigma\left(t^{\prime}, \boldsymbol{x}^{\prime}\right) h .
$$

In some cases, e.g., the non-flat spacetimes, this calculation is not practical and instead we may take $\boldsymbol{v}$ to be very small and work out $t^{\prime}$ and $\boldsymbol{x}^{\prime}$ to first order in $\boldsymbol{v}$. This approximation then gives the vector field $\xi_{P_{a}}$ generating the infinitesimal action of $P_{a}$. To be more concrete, let $X \in \mathfrak{k}$ and consider

$$
\exp (s X) \sigma(t, \boldsymbol{x})=\sigma\left(t^{\prime}, \boldsymbol{x}^{\prime}\right) h
$$

for $s$ small. Since for $s=0, t^{\prime}=t, \boldsymbol{x}^{\prime}=\boldsymbol{x}$, and $h=1$, we may write (up to $O\left(s^{2}\right)$ )

$$
\exp (s X) \sigma(t, \boldsymbol{x})=\sigma(t+s \tau, \boldsymbol{x}+s \boldsymbol{y}) \exp (Y(s))
$$

for some $Y(s) \in \mathfrak{h}$ with $Y(0)=0$, and where $\tau$ and $\boldsymbol{y}$ do not depend on $s$. Equivalently,

$$
\exp (s X) \sigma(t, \boldsymbol{x}) \exp (-Y(s))=\sigma(t+s \tau, \boldsymbol{x}+s \boldsymbol{y})
$$

again up to terms in $O\left(s^{2}\right)$. We now differentiate this equation with respect to $s$ at $s=0$. Since the equation holds up to $O\left(s^{2}\right)$, the differentiated equation is exact.

To calculate the derivative, we recall the expression for the differential of the exponential map (see, e.g., [32, § 1.2,Thm. 5])

$$
\left.\frac{d}{d s} \exp (X(s))\right|_{s=0}=\exp (X(0)) D\left(\operatorname{ad}_{X(0)}\right) X^{\prime}(0),
$$

where $D$ is the Maclaurin series corresponding to the analytic function

$$
D(z)=\frac{1-e^{-z}}{z}=1-\frac{1}{2} z+O\left(z^{2}\right) .
$$


(We have abused notation slightly and written equations as if we were working in a matrix group. This is only for clarity of exposition: the results are general.)

Let $A=t H+\boldsymbol{x} \cdot \boldsymbol{P}$. Differentiating equation (3.12), we find

$$
X \exp (A)-\exp (A) Y^{\prime}(0)=\exp (A) D\left(\operatorname{ad}_{A}\right)(\tau H+\boldsymbol{y} \cdot \boldsymbol{P})
$$

and multiplying through by $\exp (-A)$ and using that $D(z)$ is invertible as a power series with inverse the Maclaurin series corresponding to the analytic function $F(z)=z /\left(1-e^{-z}\right)$, we find

$$
G\left(\operatorname{ad}_{A}\right) X-F\left(\operatorname{ad}_{A}\right) Y^{\prime}(0)=\tau H+\boldsymbol{y} \cdot \boldsymbol{P},
$$

where we have introduced $G(z)=e^{-z} F(z)=z /\left(e^{z}-1\right)$. It is a useful observation that the analytic functions $F$ and $G$ satisfy the following relations:

$$
F(z)=K\left(z^{2}\right)+\frac{z}{2} \quad \text { and } \quad G(z)=K\left(z^{2}\right)-\frac{z}{2},
$$

for some analytic function $K(\zeta)=1+\frac{1}{12} \zeta+O\left(\zeta^{2}\right)$. To see this, simply notice that $F(z)-G(z)=z$ and that the analytic function $F(z)+G(z)$ is invariant under $z \mapsto-z$.

Equation (3.16) can now be solved for $\tau$ and $\boldsymbol{y}$ on a case by case basis. To do this, we need to compute $G\left(\operatorname{ad}_{A}\right)$ and $F\left(\operatorname{ad}_{A}\right)$ on Lie algebra elements. Often a pattern emerges which allows us to write down the result. If this fails, one can bring $\operatorname{ad}_{A}$ into Jordan normal form and then apply the usual techniques from operator calculus. A good check of our calculations is that the linear map $\mathfrak{k} \rightarrow \mathscr{X}(M)$, sending $X$ to the vector field

$$
\xi_{X}=\tau \frac{\partial}{\partial t}+y^{a} \frac{\partial}{\partial x^{a}}
$$

should be a Lie algebra anti-homomorphism: namely,

$$
\left[\xi_{X}, \xi_{Y}\right]=-\xi_{[X, Y]}
$$

We have an anti-homomorphism since the action of $\mathfrak{k}$ on $M$ is induced from the vector fields which generate left translations on $\mathcal{K}$ and these are right-invariant, hence obeying the opposite Lie algebra.

\subsection{The action of the rotations}

In this section, we illustrate the preceding discussion for the case of rotations. Here, of course, $D \geq 2$. We will see rotations act in the way we may naively expect on the exponential coordinates: namely, $t$ is a scalar and $x^{a}$ is a vector.

The infinitesimal action of the rotational generators $J_{a b}$ on the exponential coordinates can be deduced from

$$
\left[J_{a b}, H\right]=0 \quad \text { and } \quad\left[J_{a b}, P_{c}\right]=\delta_{b c} P_{a}-\delta_{a c} P_{b} .
$$

To be concrete, consider $J_{12}$, which rotates $P_{1}$ and $P_{2}$ into each other:

$$
\left[J_{12}, P_{1}\right]=-P_{2} \quad \text { and } \quad\left[J_{12}, P_{2}\right]=P_{1},
$$


but leaves $H$ and $P_{3}, \cdots, P_{D}$ inert. We see that $\operatorname{ad}_{J_{12}}^{2} P_{a}=-P_{a}$ for $a=1,2$, so that exponentiating,

$$
\begin{aligned}
\exp \left(\theta \operatorname{ad}_{J_{12}}\right)(t H+\boldsymbol{x} \cdot \boldsymbol{P})= & t H+x^{1}\left(\cos \theta P_{1}-\sin \theta P_{2}\right) \\
& +x^{2}\left(\cos \theta P_{2}+\sin \theta P_{1}\right)+x^{3} P_{3}+\cdots x^{D} P_{D} \\
= & t H+\left(x^{1} \cos \theta+x^{2} \sin \theta\right) P_{1} \\
& +\left(x^{2} \cos \theta-x^{1} \sin \theta\right) P_{2}+x^{3} P_{3}+\cdots+x^{D} P_{D} .
\end{aligned}
$$

Restricting attention to the $\left(x^{1}, x^{2}\right)$ plane, we see that the orbit of $\left(x_{0}^{1}, x_{0}^{2}\right)$ under the one-parameter subgroup $\exp \left(\theta J_{12}\right)$ of rotations is

$$
\left(\begin{array}{l}
x^{1}(\theta) \\
x^{2}(\theta)
\end{array}\right)=\left(\begin{array}{cc}
\cos \theta & \sin \theta \\
-\sin \theta & \cos \theta
\end{array}\right) \cdot\left(\begin{array}{l}
x_{0}^{1} \\
x_{0}^{2}
\end{array}\right) .
$$

Differentiating $\left(x^{1}(\theta), x^{1}(\theta)\right)$ with respect to $\theta$ yields

$$
\frac{d x^{1}}{d \theta}=x^{2} \quad \text { and } \quad \frac{d x^{2}}{d \theta}=-x^{1}
$$

so that

$$
\xi_{J_{12}}=x^{2} \frac{\partial}{\partial x^{1}}-x^{1} \frac{\partial}{\partial x^{2}}
$$

In the general case, and in the same way, we find

$$
\xi_{J_{a b}}=x^{b} \frac{\partial}{\partial x^{a}}-x^{a} \frac{\partial}{\partial x^{b}}
$$

which can be checked to obey the opposite Lie algebra

$$
\left[\xi_{J_{a b}}, \xi_{J_{c d}}\right]=-\delta_{b c} \xi_{J_{a d}}+\delta_{b d} \xi_{J_{a c}}+\delta_{a c} \xi_{J_{b d}}-\delta_{a d} \xi_{J_{b c}}=-\xi_{\left[J_{a b}, J_{c d}\right]} .
$$

\subsection{The action of the boosts}

For a homogeneous space $M=\mathcal{K} / \mathcal{H}$ of a kinematical Lie group $\mathcal{K}$ to admit a physical interpretation as a genuine spacetime, one would seem to require that the boosts act with non-compact orbits [1]. Otherwise, it would be more suitable to interpret them as (additional) rotations. In other words, if $(\mathfrak{k}, \mathfrak{h})$ is the Lie pair describing the homogeneous spacetime, with $\mathfrak{h}$ the subalgebra spanned by the rotations and the boosts, then a desirable geometrical property of $M$ is that for all $X=w^{a} B_{a} \in \mathfrak{h}$ the orbit of the one-parameter subgroup $\mathcal{B}_{X} \subset \mathcal{H}$ generated by $X$ should be homeomorphic to the real line. Of course, this requirement is strictly speaking never satisfied: the "origin" of $M$ is fixed by $\mathcal{H}$ and, in particular, by any one-parameter subgroup of $\mathcal{H}$, so its orbit under any $\mathcal{B}_{X}$ consists of just one point. Therefore the correct requirement is that the generic orbits be noncompact. It is interesting to note that we impose no such requirements on the space and time translations.

With the exception of the carrollian light cone LC, which will have to be studied separately, the action of the boosts are uniform in each class of spacetimes: lorentzian, riemannian, galilean and carrollian. (There are no boosts in aristotelian spacetimes.) We can read the action of the boosts (infinitesimally) from the Lie brackets: 
- lorentzian:

$$
[\boldsymbol{B}, H]=\boldsymbol{P}, \quad[\boldsymbol{B}, \boldsymbol{P}]=H \quad \text { and } \quad[\boldsymbol{B}, \boldsymbol{B}]=\boldsymbol{J}
$$

- riemannian:

$$
[\boldsymbol{B}, H]=-\boldsymbol{P}, \quad[\boldsymbol{B}, \boldsymbol{P}]=H \quad \text { and } \quad[\boldsymbol{B}, \boldsymbol{B}]=-\boldsymbol{J}
$$

- galilean:

$$
[\boldsymbol{B}, H]=\boldsymbol{P}
$$

- (reductive) carrollian:

$$
[\boldsymbol{B}, \boldsymbol{P}]=H
$$

- and carrollian light cone (LC):

$$
[\boldsymbol{B}, H]=-\boldsymbol{B} \quad \text { and } \quad[\boldsymbol{B}, \boldsymbol{P}]=H+\boldsymbol{J} .
$$

Below we will calculate the action of the boosts for all spacetimes except for the carrollian light cone and the exotic two-dimensional spacetimes (S17, S18, S19 $\chi$ and S20 $\chi$ ) which will be studied case by case.

In order to simplify the calculation, it is convenient to introduce two parameters $\varsigma$ and $c$ and write the infinitesimal action of the boosts as

$$
\left[B_{a}, H\right]=-\varsigma P_{a} \quad \text { and } \quad\left[B_{a}, P_{b}\right]=\frac{1}{c^{2}} \delta_{a b} H .
$$

Then $\left(\varsigma, c^{-1}\right)=(-1,1)$ for lorentzian, $\left(\varsigma, c^{-1}\right)=(1,1)$ for riemannian, $\left(\varsigma, c^{-1}\right)=(-1,0)$ for galilean and $\left(\varsigma, c^{-1}\right)=(0,1)$ for (reductive) carrollian spacetimes.

The action of the boosts on the exponential coordinates, as described in section 3.2, is given by equation (3.8), which in this case becomes

$$
t H+\boldsymbol{x} \cdot \boldsymbol{P} \mapsto \exp \left(\operatorname{ad}_{\boldsymbol{w} \cdot \boldsymbol{B}}\right)(t H+\boldsymbol{x} \cdot \boldsymbol{P}) .
$$

From equation (3.33), we see that

$$
\begin{aligned}
& \operatorname{ad}_{\boldsymbol{w} \cdot \boldsymbol{B}} H=-\varsigma \boldsymbol{w} \cdot \boldsymbol{P} \\
& \operatorname{ad}_{\boldsymbol{w} \cdot \boldsymbol{B}}^{2} H=-\frac{1}{c^{2}} \varsigma w^{2} H, \\
& \text { and } \\
& \operatorname{ad}_{\boldsymbol{w} \cdot \boldsymbol{B}} \boldsymbol{P}=\frac{1}{c^{2}} \boldsymbol{w} H \\
& \operatorname{ad}_{\boldsymbol{w} \cdot \boldsymbol{B}}^{2} \boldsymbol{P}=-\frac{1}{c^{2}} \varsigma \boldsymbol{w}(\boldsymbol{w} \cdot \boldsymbol{P}),
\end{aligned}
$$

so that in all cases $\operatorname{ad}_{\boldsymbol{w} \cdot \boldsymbol{B}}^{3}=-\frac{1}{c^{2}} \varsigma w^{2} \operatorname{ad}_{\boldsymbol{w} \cdot \boldsymbol{B}}$. This allows us to exponentiate $\operatorname{ad}_{\boldsymbol{w} \cdot \boldsymbol{B}}$ easily:

$$
\exp \left(\operatorname{ad}_{\boldsymbol{w} \cdot \boldsymbol{B}}\right)=1+\frac{\sinh z}{z} \operatorname{ad}_{\boldsymbol{w} \cdot \boldsymbol{B}}+\frac{\cosh z-1}{z^{2}} \operatorname{ad}_{\boldsymbol{w} \cdot \boldsymbol{B}}^{2},
$$

where $z^{2}=-\frac{1}{c^{2}} \varsigma w^{2}$, and hence

$$
\begin{aligned}
\exp \left(\operatorname{ad}_{\boldsymbol{w} \cdot \boldsymbol{B}}\right) t H & =t \cosh z H-\varsigma t \frac{\sinh z}{z} \boldsymbol{w} \cdot \boldsymbol{P} \\
\exp \left(\operatorname{ad}_{\boldsymbol{w} \cdot \boldsymbol{B}}\right) \boldsymbol{x} \cdot \boldsymbol{P} & =\boldsymbol{x} \cdot \boldsymbol{P}+\frac{1}{c^{2}} \frac{\sinh z}{z} \boldsymbol{x} \cdot \boldsymbol{w} H+\frac{\cosh z-1}{w^{2}}(\boldsymbol{x} \cdot \boldsymbol{w}) \boldsymbol{w} \cdot \boldsymbol{P} .
\end{aligned}
$$


Therefore, the orbit of $\left(t_{0}, \boldsymbol{x}_{0}\right)$ under $\exp (s \boldsymbol{w} \cdot \boldsymbol{B})$ is given by

$$
\begin{aligned}
& t(s)=t_{0} \cosh (s z)+\frac{1}{c^{2}} \frac{\sinh (s z)}{z} \boldsymbol{x}_{0} \cdot \boldsymbol{w}, \\
& \boldsymbol{x}(s)=\boldsymbol{x}_{0}^{\perp}-\varsigma t_{0} \frac{\sinh (s z)}{z} \boldsymbol{w}+\frac{\cosh (s z)}{w^{2}}\left(\boldsymbol{x}_{0} \cdot \boldsymbol{w}\right) \boldsymbol{w},
\end{aligned}
$$

where we have introduced $\boldsymbol{x}_{0}^{\perp}:=\boldsymbol{x}_{0}-\frac{\boldsymbol{x}_{0} \cdot \boldsymbol{w}}{w^{2}} \boldsymbol{w}$ to be the component of $\boldsymbol{x}_{0}$ perpendicular to $\boldsymbol{w}$. It follows from this expression that $\boldsymbol{x}^{\perp}(s)=\boldsymbol{x}_{0}^{\perp}$, so that the orbit lies in a plane spanned by $\boldsymbol{w}$ and the time direction.

Differentiating these expressions with respect to $s$, we arrive at the fundamental vector field $\xi_{B_{a}}$. Indeed, differentiating $(t(s), \boldsymbol{x}(s))$ with respect to $s$ at $s=0$, we obtain the value of $\xi_{\boldsymbol{w} \cdot \boldsymbol{B}}$ at the point $\left(t_{0}, x_{0}\right)$. Letting $\left(t_{0}, x_{0}\right)$ vary we obtain that

$$
\xi_{B_{a}}=\frac{1}{c^{2}} x^{a} \frac{\partial}{\partial t}-\varsigma t \frac{\partial}{\partial x^{a}} .
$$

In particular, notice that one of the virtues of the exponential coordinates, is that the fundamental vector fields of the stabiliser $\mathfrak{h}$ - that is, of the rotations and the boosts - are linear and, in particular, they are complete. This will be useful in determining whether or not the generic orbits of one-parameter subgroup of boosts are compact.

Let $\exp (s \boldsymbol{w} \cdot \boldsymbol{B}), s \in \mathbb{R}$, be a one-parameter subgroup consisting of boosts. Given any $p \in M$, its orbit under this subgroup is the image of the map $c: \mathbb{R} \rightarrow M$, where $c(s):=\exp (s \boldsymbol{w} \cdot \boldsymbol{B}) \cdot p$. As we just saw, in the reductive examples (all but LC) the fundamental vector field $\xi_{\boldsymbol{w} \cdot \boldsymbol{B}}$ is linear in the exponential coordinates, and hence it is complete. Therefore, its integral curves are one-dimensional connected submanifolds of $M$ and hence either homeomorphic to the real line (if non compact) or to the circle (if compact). The compact case occurs if and only if the map $c$ is periodic.

If the exponential coordinates define a global coordinate chart (which means, in particular, that the homogeneous space is diffeomorphic to $\mathbb{R}^{D+1}$ ), then it is only a matter of solving a linear ODE to determine whether or not $c$ is periodic. In any case, we can determine whether or not this is the case in the exponential coordinate chart centred at the origin. For the special case of symmetric spaces, which are the spaces obtained via limits from the riemannian and lorentzian maximally symmetric spaces, we may use Theorem 1 , which gives an infinitesimal criterion for when the exponential coordinates define a global chart. Recalling the discussion in section 3.1 , we again state that $\mathbb{M}, \mathbb{E}, \mathbb{H}, \mathrm{G}, \mathrm{dSG}, \mathrm{C}$, and AdSC satisfy criterion (3) in Theorem 1 and hence that the exponential coordinates define a diffeomorphism $M \cong \mathbb{R}^{D+1}$. Using exponential coordinates, we will see that the orbits of boosts in $\mathbb{E}$ and $\mathbb{H}$ are compact, whereas the generic orbits of boosts in the other cases are non-compact.

The remaining symmetric spacetimes dS, AdS, $\mathbb{S}$, AdSG, and dSC do not satisfy the infinitesimal criterion (3) in Theorem 1, and hence the exponential coordinates are not a global chart. It may nevertheless still be the case that the image of $\exp _{o}$ covers the homogeneous spacetime (or a dense subset). It turns out that $\mathbb{S}$ is exponential and AdSG is weakly exponential. The result for $\mathbb{S}$ is classical, since the sphere is a compact riemannian symmetric space, and the case of AdSG follows from Theorem 2. If $D \leq 2$, then the 
kinematical Lie group for AdSG is solvable and hence AdSG is weakly exponential, whereas if $D \geq 3$, the radicals $\operatorname{rad} \mathfrak{k}=\operatorname{span}\{\boldsymbol{B}, \boldsymbol{P}, H\}$ and $\operatorname{rad} \mathfrak{h}=\operatorname{span}\{\boldsymbol{B}\}$. Therefore, $\mathfrak{k} / \operatorname{rad} \mathfrak{k} \cong$ $\mathfrak{s o}(D) \cong \mathfrak{h} / \operatorname{rad} \mathfrak{h}$. Therefore, with $\widehat{\mathcal{K}}:=\mathcal{K} / \operatorname{Rad}(\mathcal{K})$ and similarly for $\widehat{\mathcal{H}}, \widehat{\mathcal{K}} / \widehat{\mathcal{H}}$ is trivially weakly exponential and hence, by Theorem 2 , so is $\mathcal{K} / \mathcal{H}$. We will see that boosts act with compact orbits in $\mathbb{S}$, but with non-compact orbits in AdSG.

Among the symmetric spaces in table 1, this leaves dS, AdS, and dSC. We treat those cases using the same technique, which will also work for the non-symmetric LC. Let $M$ be a simply-connected homogeneous spacetime and $q: M \rightarrow \bar{M}$ a covering map which is equivariant under the action of (the universal covering group of) $\mathcal{K}$. By equivariance, $q(\exp (s \boldsymbol{w} \cdot \boldsymbol{B}) \cdot o)=\exp (s \boldsymbol{w} \cdot \boldsymbol{B}) \cdot q(o)$, so the orbit of $o \in M$ under the boost is sent by $q$ to the orbit of $q(o) \in \bar{M}$. Since $q$ is continuous it sends compact sets to compact sets, so if the orbit of $q(o) \in \bar{M}$ is not compact then neither is the orbit of $o \in M$. For $M$ one of dS, AdS, dSC, or LC, there is some covering $q: M \rightarrow \bar{M}$ such that we can equivariantly embed $\bar{M}$ as a hypersurface in some pseudo-euclidean space where $\mathcal{K}$ acts linearly. It is a simple matter to work out the nature of the orbits of the boosts in the ambient pseudo-euclidean space (and hence on $\bar{M}$ ), with the caveat that what is a boost in $\bar{M}$ need not be a boost in the ambient space. Having shown that the boost orbit is non-compact on $\bar{M}$ we deduce that the orbit is non-compact on $M$. We will show in this way that the generic boost orbits are non-compact for dS, AdS, dSC, and LC.

Finally, this still leaves the torsional galilean spacetimes $\mathrm{dSG}_{\gamma}, \mathrm{AdSG}_{\chi}$ and $\mathrm{S}_{2} 2_{\gamma, \chi}$, which require a different argument to be explained when we discuss these spacetimes in section 6.5.

\subsection{Invariant connections}

There is only one non-reductive homogeneous spacetime in table 1 and 2, namely LC, and its invariant connections were already determined in [6]. There it is shown the light cone for $D \geq 2$ admits no invariant connections, whereas for $D=1$ there is a three-parameter family of invariant connections and a unique torsion-free, flat connection. We will, therefore, restrict ourselves to the remaining reductive homogeneous spaces in this section.

Let $(\mathfrak{k}, \mathfrak{h})$ be a Lie pair associated to a reductive homogeneous space. We assume that $(\mathfrak{k}, \mathfrak{h})$ is effective so that $\mathfrak{h}$ does not contain any non-zero ideals of $\mathfrak{k}$. We let $\mathfrak{k}=\mathfrak{h} \oplus \mathfrak{m}$ denote a reductive split, where $[\mathfrak{h}, \mathfrak{m}] \subset \mathfrak{m}$. This split makes $\mathfrak{m}$ into an $\mathfrak{h}$-module relative to the linear isotropy representation $\lambda: \mathfrak{h} \rightarrow \mathfrak{g l}(\mathfrak{m})$, where

$$
\lambda_{X} Y=[X, Y] \quad \text { for all } X \in \mathfrak{h} \text { and } Y \in \mathfrak{m} .
$$

As shown in [33], one can uniquely characterise the invariant affine connections on $(\mathfrak{k}, \mathfrak{h})$ by their Nomizu map $\alpha: \mathfrak{m} \times \mathfrak{m} \rightarrow \mathfrak{m}$, an $\mathfrak{h}$-equivariant bilinear map; that is, such that for all $X \in \mathfrak{h}$ and $Y, Z \in \mathfrak{m}$,

$$
[X, \alpha(Y, Z)]=\alpha([X, Y], Z)+\alpha(Y,[X, Z]) .
$$

The torsion and curvature of an invariant affine connection with Nomizu map $\alpha$ are given, respectively, by the following expressions for all $X, Y, Z \in \mathfrak{m}$,

$$
\begin{aligned}
\Theta(X, Y) & =\alpha(X, Y)-\alpha(Y, X)-[X, Y]_{\mathfrak{m}}, \\
\Omega(X, Y) Z & =\alpha(X, \alpha(Y, Z))-\alpha(Y, \alpha(X, Z))-\alpha\left([X, Y]_{\mathfrak{m}}, Z\right)-\left[[X, Y]_{\mathfrak{h}}, Z\right],
\end{aligned}
$$


where $[X, Y]=[X, Y]_{\mathfrak{h}}+[X, Y]_{\mathfrak{m}}$ is the decomposition of $[X, Y] \in \mathfrak{k}=\mathfrak{h} \oplus \mathfrak{m}$. In particular, for the canonical invariant connection with zero Nomizu map, we have

$$
\Theta(X, Y)=-[X, Y]_{\mathfrak{m}} \quad \text { and } \quad \Omega(X, Y) Z=-\lambda_{[X, Y]_{\mathfrak{h}}} Z .
$$

For kinematical homogeneous spacetimes, we can determine the possible Nomizu maps in a rather uniform way. Rotational invariance determines the form of the Nomizu map up to a few parameters and then we need only study the action of the boosts. From table 1 it is clear that the action of the boosts is common to all spacetimes within a given class: lorentzian, riemannian, galilean, and carrollian; although the curvature and torsion of the invariant connections of course do depend on the spacetime in question.

\subsection{The soldering form and the canonical connection}

Recall that on the Lie group $\mathcal{K}$ there is a left-invariant $\mathfrak{k}$-valued one-form $\vartheta$ : the (leftinvariant) Maurer-Cartan one-form. It obeys the structure equation

$$
d \vartheta=-\frac{1}{2}[\vartheta, \vartheta]
$$

where the notation hides the wedge product in the right-hand side. Using exponential coordinates, we can pull back $\vartheta$ to a neighbourhood of the origin on $M$. The following formula, which follows from equation (3.13), shows how to calculate it:

$$
\sigma^{*} \vartheta=D\left(\operatorname{ad}_{A}\right)(d t H+d \boldsymbol{x} \cdot \boldsymbol{P}),
$$

where, as before, $A=t H+\boldsymbol{x} \cdot \boldsymbol{P}$ and $D$ is the Maclaurin series corresponding to the analytic function in (3.14).

The pull-back $\sigma^{*} \vartheta$ is a one-form defined near the origin on $M$ with values in the Lie algebra $\mathfrak{k}$. Let $\mathfrak{m}$ be a vector space complement to $\mathfrak{h}$ in $\mathfrak{k}$ so that as a vector space $\mathfrak{k}=\mathfrak{h} \oplus \mathfrak{m}$. This split allows us to write

$$
\sigma^{*} \vartheta=\theta+\omega,
$$

where $\theta$ is $\mathfrak{m}$-valued and and $\omega$ is $\mathfrak{h}$-valued. If the Lie pair $(\mathfrak{k}, \mathfrak{h})$ is reductive and $\mathfrak{m}$ is chosen to be an $\mathfrak{h}$-submodule of $\mathfrak{k}$, then $\omega$ is the one-form corresponding to the canonical invariant connection on $M$. The soldering form is then given by $\theta$.

The torsion and curvature of $\omega$ are easy to calculate using the fact that $\vartheta$ obeys the Maurer-Cartan structure equation (3.44). ${ }^{6}$ Indeed, the torsion two-form $\Theta$ is given by

$$
\Theta=d \theta+[\omega, \theta]=-\frac{1}{2}[\theta, \theta]_{\mathfrak{m}}
$$

and the curvature two-form $\Omega$ by

$$
\Omega=d \omega+\frac{1}{2}[\omega, \omega]=-\frac{1}{2}[\theta, \theta]_{\mathfrak{h}},
$$

which agree with the expressions in equation (3.43).

\footnotetext{
${ }^{6}$ Let us emphasise that in this work, curvature always refer to the curvature of an invariant affine connection and hence should not be confused with the curvature of the associated Cartan connection, which is always flat for the homogeneous spaces (also called Klein geometries in that context).
} 
In the non-reductive case $\omega$ does not define a connection, but we may still project the locally defined $\mathfrak{k}$-valued one-form $\sigma^{*} \vartheta$ to $\mathfrak{k} / \mathfrak{h}$. The resulting local one-form $\theta$ with values in $\mathfrak{k} / \mathfrak{h}$ is a soldering form which defines an isomorphism $T_{p} M \rightarrow \mathfrak{k} / \mathfrak{h}$ for every $p \in M$ near the origin. Wherever $\theta$ is invertible, the exponential coordinates define an immersion, which may however fail to be an embedding or indeed even injective. In practice, it is not easy to determine injectivity, but it is easy to determine where $\theta$ is invertible by calculating the top exterior power of $\theta$ and checking that it is non-zero. Provided that $\theta$ is invertible, the inverse isomorphism is the vielbein $E$, where $E(p): \mathfrak{k} / \mathfrak{h} \rightarrow T_{p} M$ for every $p \in M$ near the origin. The vielbein allows us to transport tensors on $\mathfrak{k} / \mathfrak{h}$ to tensor fields on $M$ and, as we now recall, it takes $\mathcal{H}$-invariant tensors on $\mathfrak{k} / \mathfrak{h}$ to $\mathcal{K}$-invariant tensor fields on $M$.

\subsection{Invariant tensors}

It is well-known that $\mathcal{K}$-invariant tensor fields on $M=\mathcal{K} / \mathcal{H}$ are in one-to-one correspondence with $\mathcal{H}$-invariant tensors on $\mathfrak{k} / \mathfrak{h}$ and if $\mathcal{H}$ is connected, with $\mathfrak{h}$-invariant tensors on $\mathfrak{k} / \mathfrak{h}$. We may assume that $\mathcal{H}$ is indeed connected, passing to the universal cover of $M$, if necessary. In practice, given an $(r, s)$-tensor $T$ on $\mathfrak{k} / \mathfrak{h}$ - that is, an element of $(\mathfrak{k} / \mathfrak{h})^{\otimes r} \otimes\left((\mathfrak{k} / \mathfrak{h})^{*}\right)^{\otimes s}$ - we can turn it into an $(r, s)$-tensor field $\mathscr{T}$ on $M$ by contracting with soldering forms and vielbeins as appropriate to arrive, for every $p \in M$, to $\mathscr{T}(p) \in\left(T_{p} M\right)^{\otimes r} \otimes\left(T_{p}^{*} M\right)^{\otimes s}$. Moreover, if $T$ is $\mathcal{H}$-invariant, $\mathscr{T}$ is $\mathcal{K}$-invariant.

Our choice of basis for $\mathfrak{k}$ is such that $\boldsymbol{J}$ and $\boldsymbol{B}$ span $\mathfrak{h}$ and therefore $\overline{\boldsymbol{P}}:=\boldsymbol{P} \bmod \mathfrak{h}$ and $\bar{H}:=H \bmod \mathfrak{h}$ span $\mathfrak{k} / \mathfrak{h}$. In the reductive case, $\mathfrak{k}=\mathfrak{h} \oplus \mathfrak{m}$ and $\mathfrak{m} \cong \mathfrak{k} / \mathfrak{h}$ as $\mathfrak{h}$-modules. We will let $\eta$ and $\pi^{a}$ denote the canonical dual basis for $(\mathfrak{k} / \mathfrak{h})^{*}$.

Invariant non-degenerate metrics are in one-to-one correspondence with $\mathfrak{h}$-invariant non-degenerate symmetric bilinear forms on $\mathfrak{k} / \mathfrak{h}$ and characterise, depending on their signature, lorentzian or riemannian spacetimes. On the other hand, invariant galilean structures $^{7}$ consist of a pair $(\tau, h)$, where $\tau \in(\mathfrak{k} / \mathfrak{h})^{*}$ and $h \in S^{2}(\mathfrak{k} / \mathfrak{h})$ are $\mathfrak{h}$-invariant, $h$ has co-rank 1 and $h(\tau,-)=0$, if we think of $h$ as a symmetric bilinear form on $(\mathfrak{k} / \mathfrak{h})^{*}$. On $M, \tau$ gives rise to an invariant clock one-form and $h$ to an invariant spatial metric on one-forms. Carrollian structures are dual to galilean structures and consist of a pair $(\kappa, b)$, where $\kappa \in \mathfrak{k} / \mathfrak{h}$ defines an invariant vector field and $b \in S^{2}(\mathfrak{k} / \mathfrak{h})^{*}$ is an invariant symmetric bilinear form of co-rank 1 and such that $b(\kappa,-)=0$. Homogeneous aristotelian spacetimes admit an invariant galilean structure and an invariant carrollian structure simultaneously.

Invariance under $\mathfrak{h}$ implies, in particular, invariance under the rotational subalgebra, which is non-trivial for $D \geq 2$. Assuming that $D \geq 2$ for now, it is easy to write down the possible rotationally invariant tensors and therefore we need only check invariance under $\boldsymbol{B}$. The action of $\boldsymbol{B}$ is induced by duality from the action on $\mathfrak{k} / \mathfrak{h}$ which is given by

$$
\lambda_{B_{a}}(\bar{H})=\overline{\left[B_{a}, H\right]} \quad \text { and } \quad \lambda_{B_{a}}\left(\bar{P}_{b}\right)=\overline{\left[B_{a}, P_{b}\right]}
$$

with the brackets being those of $\mathfrak{k}$. In practice, we can determine this from the explicit expression of the Lie brackets by computing the brackets in $\mathfrak{k}$ and simply dropping any $\boldsymbol{B}$ or $\boldsymbol{J}$ from the right-hand side. The only possible invariants in $\mathfrak{k} / \mathfrak{h}$ are proportional to $H$,

\footnotetext{
${ }^{7}$ We will not distinguish notationally the $\mathcal{H}$-invariant tensor from the $\mathcal{K}$-invariant tensor field.
} 
which is invariant provided that $[\boldsymbol{B}, H]=0 \bmod \mathfrak{h}$. Dually, the only possible invariants in $(\mathfrak{k} / \mathfrak{h})^{*}$ are proportional to $\eta$, which is invariant provided that there is no $X \in \mathfrak{k}$ such that $H$ appears in $[\boldsymbol{B}, X]$. Omitting the tensor product symbol, the only rotational invariants in $S^{2}(\mathfrak{k} / \mathfrak{h})$ are linear combinations of $H^{2}$ and $P^{2}:=\delta^{a b} P_{a} P_{b}$, whereas in $S^{2}(\mathfrak{k} / \mathfrak{h})^{*}$ are $\eta^{2}$ and $\pi^{2}=\delta_{a b} \pi^{a} \pi^{b}$.

In $D=1$ there are no rotations, so we need only concern ourselves with the action of $\boldsymbol{B}$. Possible invariants in $\mathfrak{k} / \mathfrak{h}$ are linear combinations of $H$ and $\boldsymbol{P}$, whereas in $(\mathfrak{k} / \mathfrak{h})^{*}$ they are linear combinations of $\eta$ and $\pi$. Similarly in the space of symmetric tensors, we can have now linear combinations of $H^{2}, H \boldsymbol{P}$, and $\boldsymbol{P}^{2}$ in $S^{2}(\mathfrak{k} / \mathfrak{h})$ and of $\eta^{2}, \eta \pi$, and $\pi^{2}$ in $S^{2}(\mathfrak{k} / \mathfrak{h})^{*}$. These are again easy to determine from the Lie bracket.

\section{Invariant connections, curvature, and torsion for reductive spacetimes}

In this section we determine the invariant affine connections for the reductive spacetimes in tables 1 and 2. This is equivalent to determining the space of Nomizu maps which, as explained above, can be done uniformly, a class at a time. We also calculate the curvature and torsion of the invariant connections.

For reductive homogeneous spaces there always exists, besides the canonical connection with vanishing Nomizu map, another interesting connection. It is given by the torsion-free connection defined $^{8}$ by $\alpha(X, Y)=\frac{1}{2}[X, Y]_{\mathfrak{m}}$. The canonical and the natural torsion-free connections have the same geodesics and, as one can easily observe below, the connections coincide for symmetric spaces.

For any spacetime the Nomizu maps needs to be rotationally invariant which gives us

$$
\begin{aligned}
\alpha(H, H) & =\left\{\begin{array}{lll}
\mu H & D>1 \\
\mu H+\mu^{\prime} P & D=1
\end{array}\right. \\
\alpha\left(P_{a}, P_{b}\right)= \begin{cases}\zeta \delta_{a b} H & \left.\alpha>3, P_{a}\right)= \begin{cases}\nu P_{a} & D>2 \\
\nu P_{a}+\nu^{\prime} \epsilon_{a b} P_{b} & D=2 \\
\nu P+\nu^{\prime} H & D=1\end{cases} \\
\zeta \delta_{a b} H+\zeta^{\prime} \epsilon_{a b c} P_{c} & D=3 \\
\zeta \delta_{a b} H+\zeta^{\prime} \epsilon_{a b} H & D=2 \\
\zeta H+\zeta^{\prime} P & D=1\end{cases} & \alpha\left(P_{a}, H\right)= \begin{cases}\xi P_{a} & D>2 \\
\xi P_{a}+\xi^{\prime} \epsilon_{a b} P_{b} & D=2 \\
\xi P+\xi^{\prime} H & D=1,\end{cases}
\end{aligned}
$$

for some real parameters $\mu, \mu^{\prime}, \nu, \nu^{\prime}, \zeta, \zeta^{\prime}, \xi, \xi^{\prime}$. Now we simply impose invariance under $B_{a}$.

\subsection{Nomizu maps for lorentzian spacetimes}

The lorentzian spacetimes in table 1 all share the same action of the boosts:

$$
\lambda_{B_{a}} H=P_{a} \quad \text { and } \quad \lambda_{B_{a}} P_{b}=\delta_{a b} H
$$

We will impose invariance explicitly in this case to illustrate the calculation and only state the results in all other cases.

\footnotetext{
${ }^{8}$ It is the unique Nomizu map with $\alpha(X, X)=0$ for all $X \in \mathfrak{m}$ and vanishing torsion and called "canonical affine connection of the first kind" in [33].
} 


\subsection{1 $D \geq 4$}

We calculate (remember (3.41))

$$
\left(\lambda_{B_{c}} \alpha\right)\left(P_{a}, P_{b}\right)=\zeta \delta_{a b} P_{c}-\nu \delta_{a c} P_{b}-\xi \delta_{b c} P_{a},
$$

whose vanishing requires $\zeta=\nu=\xi=0$, as can be seen by considering $a=b \neq c, a=c \neq b$, and $b=c \neq a$ in turn. Finally,

$$
\left(\lambda_{B_{c}} \alpha\right)(H, H)=\mu P_{c},
$$

whose vanishing imposes $\mu=0$ and hence the only invariant Nomizu map is the zero map.

\subsection{2 $D=3$}

The only change here is an additional term $\zeta^{\prime} \epsilon_{a b c} P_{c}$ in $\alpha\left(P_{a}, P_{b}\right)$. This results in

$$
\left(\lambda_{B_{c}} \alpha\right)\left(P_{a}, P_{b}\right)=\zeta \delta_{a b} P_{c}+\zeta^{\prime} \epsilon_{a b c} H-\nu \delta_{a c} P_{b}-\xi \delta_{b c} P_{a},
$$

whose vanishing again requires $\zeta=\zeta^{\prime}=\nu=\xi=0$. Hence continuing as in $D \geq 4$, we find that the only invariant Nomizu map is the zero map.

\subsection{3 $D=2$}

The $2+1$ dimensional case differs with respect to its higher dimensional counterparts. We start by calculating

$$
\left(\lambda_{B_{c}} \alpha\right)\left(P_{a}, P_{b}\right)=\zeta \delta_{a b} P_{c}+\zeta^{\prime} \epsilon_{a b} P_{c}-\delta_{a c}\left(\nu P_{b}+\nu^{\prime} \epsilon_{b d} P_{d}\right)-\delta_{b c}\left(\xi P_{a}+\xi^{\prime} \epsilon_{a d} P_{d}\right) .
$$

Considering $a=b \neq c$ requires that $\zeta=0$. Next we set $a=c \neq b$, which leads us to $\nu=0$ and $\zeta^{\prime}=-\nu^{\prime}$. Similarly, $b=c \neq a$ imposes $\xi=0$ and $\zeta^{\prime}=\xi^{\prime}$ and leaves us, for now, with $t=\zeta^{\prime}=\xi^{\prime}=-\nu^{\prime}$. We need to check if the remaining components of the Nomizu map are also invariant, e.g.,

$$
\left(\lambda_{B_{c}} \alpha\right)(H, H)=\mu P_{c}
$$

vanishes if and only if $\mu=0$, while $\alpha\left(P_{a}, H\right)$ and $\alpha\left(H, P_{a}\right)$ are invariant without further ado.

In summary, we get a one-parameter family of Nomizu maps, parametrised by $t \in \mathbb{R}$,

$$
\alpha\left(P_{a}, P_{b}\right)=t \epsilon_{a b} H \quad \alpha\left(H, P_{a}\right)=-t \epsilon_{a b} P_{b} \quad \alpha\left(P_{a}, H\right)=t \epsilon_{a b} P_{b} .
$$

It can be written in a more compact way using lorentzian $2+1$ dimensional tensors, $\alpha\left(P_{\mu}, P_{\nu}\right)=\tilde{t} \epsilon_{\mu \nu \rho} \eta^{\rho \sigma} P_{\sigma}$.

\subsection{4 $D=1$}

Here we notice that $\lambda_{B}$ is the identity on $\mathfrak{m}$, hence minus the identity on $\mathfrak{m}^{*}$. Therefore, by parity, there are no zero eigenvalues in $\mathfrak{m}^{*} \otimes \mathfrak{m}^{*} \otimes \mathfrak{m}$ and hence no invariants but the zero Nomizu map.

In summary, lorentzian homogeneous spacetimes have, with the exception $D=2$, of a unique invariant connection given by the canonical connection. As we will see in the next sections, there is more freedom for galilean and carrollian spacetimes. However since we start with an unique (vanishing) Nomizu map, only this vanishing case arises also as a limit. The additional invariant non-relativistic and ultra-relativistic connections can be seen as an intrinsic property that does not originate from the relativistic spacetimes. 


\subsection{Nomizu maps for riemannian spacetimes}

The situation here is very similar to the lorentzian case. Now the boosts act as

$$
\lambda_{B_{a}} H=-P_{a} \quad \text { and } \quad \lambda_{B_{a}} P_{b}=\delta_{a b} H .
$$

The results are as in the lorentzian case: the only invariant connection is the canonical connection, except in $D=2$ where there is a one-parameter family.

\subsection{Nomizu maps for galilean spacetimes}

On a galilean spacetime, the boosts act as

$$
\lambda_{B_{a}} H=P_{a}
$$

and the $P_{a}$ are invariant. This results in the following invariant Nomizu maps:

$$
\begin{array}{cl}
\alpha(H, H)=\left\{\begin{array}{lll}
(\nu+\xi) H & D>1 \\
(\nu+\xi) H+\mu^{\prime} P & D=1
\end{array}\right. & \alpha\left(H, P_{a}\right)= \begin{cases}\nu P_{a} & D \neq 2 \\
\nu P_{a}+\nu^{\prime} \epsilon_{a b} P_{b} & D=2\end{cases} \\
\alpha\left(P_{a}, P_{b}\right)=0 & \alpha\left(P_{a}, H\right)= \begin{cases}\xi P_{a} & D \neq 2 \\
\xi P_{a}-\nu^{\prime} \epsilon_{a b} P_{b} & D=2 .\end{cases}
\end{array}
$$

We will now analyse the curvature and torsion for these Nomizu maps for each galilean spacetime.

\subsubsection{Galilean spacetime (G)}

For $D \geq 3$, the torsion and curvature of the resulting connection have the following non-zero components:

$$
\Theta\left(H, P_{a}\right)=(\nu-\xi) P_{a} \quad \text { and } \quad \Omega\left(H, P_{a}\right) H=-\xi^{2} P_{a} .
$$

There is a unique torsion-free, flat invariant connection corresponding to the canonical connection with $\nu=\xi=0$.

For $D=2$, the torsion and curvature are given by the following non-zero components:

$$
\Theta\left(H, P_{a}\right)=(\nu-\xi) P_{a}+2 \nu^{\prime} \epsilon_{a b} P_{b} \quad \text { and } \quad \Omega\left(H, P_{a}\right) H=\left(\nu^{\prime 2}-\xi^{2}\right) P_{a}+2 \nu^{\prime} \xi \epsilon_{a b} P_{b}
$$

so that again the canonical connection is the unique torsion-free, flat invariant connection.

Finally, for $D=1$, torsion and curvature are given by

$$
\Theta(H, P)=(\nu-\xi) P \quad \text { and } \quad \Omega(H, P) H=-\xi^{2} P .
$$

Since neither depend on $\mu^{\prime}$, we now have a one-parameter family of torsion-free, flat invariant connections, defined by the Nomizu map

$$
\alpha(H, H)=\mu^{\prime} P .
$$




\subsubsection{Galilean de Sitter spacetime (dSG)}

Let $D \geq 3$. The torsion and curvature, given by equation (3.42), have the following nonvanishing components:

$$
\Theta\left(H, P_{a}\right)=(\nu-\xi) P_{a} \quad \text { and } \quad \Omega\left(H, P_{a}\right) H=\left(1-\xi^{2}\right) P_{a} .
$$

Therefore, there are two torsion-free, flat invariant connections corresponding to $\nu=\xi=$ \pm 1 . The Nomizu maps for these two connections are

$$
\begin{aligned}
\alpha(H, H) & =2 H \\
\alpha\left(H, P_{a}\right) & =P_{a} \\
\alpha\left(P_{a}, H\right) & =P_{a}
\end{aligned} \quad \text { and } \quad \begin{aligned}
\alpha(H, H) & =-2 H \\
\alpha\left(H, P_{a}\right) & =-P_{a} \\
\alpha\left(P_{a}, H\right) & =-P_{a} .
\end{aligned}
$$

In $D=2$, the vector space of Nomizu maps is three-dimensional and the non-vanishing curvature and torsion components in this dimension are

$$
\Theta\left(H, P_{a}\right)=(\nu-\xi) P_{a}+2 \nu^{\prime} \epsilon_{a b} P_{b} \quad \text { and } \quad \Omega\left(H, P_{a}\right) H=\left(\nu^{\prime 2}-\xi^{2}+1\right) P_{a}+2 \xi \nu^{\prime} \epsilon_{a b} P_{b} \text {. }
$$

Again, there are two torsion-free, flat invariant connection corresponding to $\nu=\xi= \pm 1$.

Finally, let $D=1$. The non-vanishing torsion and curvature components are

$$
\Theta(H, P)=(\nu-\xi) P \quad \text { and } \quad \Omega(H, P) H=\left(1-\xi^{2}\right) P .
$$

The torsion-free, flat connections are once again given by $\nu=\xi= \pm 1$, but now there is a free parameter $\mu^{\prime}$.

\subsubsection{Galilean anti de Sitter spacetime (AdSG)}

The torsion and curvature have have the following non-zero components:

$$
\Theta\left(H, P_{a}\right)= \begin{cases}(\nu-\xi) P_{a} & D \neq 2 \\ (\nu-\xi) P_{a}+2 \nu^{\prime} \epsilon_{a b} P_{b} & D=2\end{cases}
$$

and

$$
\Omega\left(H, P_{a}\right) H= \begin{cases}-\left(1+\xi^{2}\right) P_{a} & D \neq 2 \\ -\left(1+\xi^{2}-\nu^{\prime 2}\right) P_{a}+2 \xi \nu^{\prime} \epsilon_{a b} P_{b} & D=2 .\end{cases}
$$

There are torsion-free connections, but none are flat.

\subsubsection{Torsional galilean de Sitter spacetime $\left(\mathrm{dSG}_{\gamma=1}\right)$}

Let $D \geq 3$. The torsion has the following non-zero components

$$
\Theta\left(H, P_{a}\right)=(\nu-\xi-2) P_{a},
$$

whereas the only non-zero component of the curvature is

$$
\Omega\left(H, P_{a}\right) H=-(1+\xi)^{2} P_{a} .
$$


Therefore, there exists a unique invariant connection with zero torsion and curvature corresponding to $\nu=1$ and $\xi=-1$ :

$$
\alpha\left(H, P_{a}\right)=P_{a} \quad \text { and } \quad \alpha\left(P_{a}, H\right)=-P_{a} .
$$

If $D=2$, we have an additional parameter in our family of invariant affine connections:

$$
\alpha(H, H)=(\nu+\xi) H, \quad \alpha\left(H, P_{a}\right)=\nu P_{a}+\nu^{\prime} \epsilon_{a b} P_{b}, \quad \text { and } \quad \alpha\left(P_{a}, H\right)=\xi P_{a}-\nu^{\prime} \epsilon_{a b} P_{b} .
$$

The only non-zero component of the torsion is

$$
\Theta\left(H, P_{a}\right)=(\nu-\xi-2) P_{a}+2 \nu^{\prime} \epsilon_{a b} P_{b},
$$

and the only non-zero component of the curvature is

$$
\Omega\left(H, P_{a}\right) H=\left(\left(\nu^{\prime}\right)^{2}-(1+\xi)^{2}\right) P_{a}+2 \nu^{\prime}(1+\xi) \epsilon_{a b} P_{b} .
$$

We see that there is a unique torsion-free, flat invariant connection with Nomizu map

$$
\alpha\left(H, P_{a}\right)=P_{a} \quad \text { and } \quad \alpha\left(P_{a}, H\right)=-P_{a} .
$$

Finally, in $D=1$ we have a three-parameter family of Nomizu maps:

$$
\alpha(H, H)=(\nu+\xi) H+\mu^{\prime} P, \quad \alpha(H, P)=\nu P, \quad \text { and } \quad \alpha(P, H)=\xi P .
$$

The torsion is given by

$$
\Theta(H, P)=(\nu-\xi-2) P
$$

and the curvature by

$$
\Omega(H, P) H=-(1+\xi)^{2} P .
$$

Imposing zero torsion and zero curvature still leaves a one-parameter family of invariant connections with Nomizu map

$$
\alpha(H, H)=\mu^{\prime} P, \quad \alpha(H, P)=P, \quad \text { and } \quad \alpha(P, H)=-P .
$$

\subsubsection{Torsional galilean de Sitter spacetime $\left(\mathrm{dSG}_{\gamma \neq 1}\right)$}

For $D \geq 3$, the torsion is given by

$$
\Theta\left(H, P_{a}\right)=(\nu-\xi-(1+\gamma)) P_{a}
$$

and the curvature by

$$
\Omega\left(H, P_{a}\right) H=-(\xi+1)(\xi+\gamma) P_{a} .
$$

Therefore, there are precisely two torsion-free, flat invariant connections, with Nomizu maps

$$
\begin{aligned}
& \alpha(H, H)=(\gamma-1) H \\
& \alpha(H, H)=(1-\gamma) H \\
& \alpha\left(H, P_{a}\right)=\gamma P_{a} \\
& \text { and } \\
& \alpha\left(H, P_{a}\right)=P_{a} \\
& \alpha\left(P_{a}, H\right)=-P_{a} \\
& \alpha\left(P_{a}, H\right)=-\gamma P_{a} \text {. }
\end{aligned}
$$


If $D=2$, then there is a three-parameter family of invariant connections with torsion and curvature that have the following non-zero components:

$$
\begin{aligned}
\Theta\left(H, P_{a}\right) & =(\nu-\xi-(1+\gamma)) P_{a}+2 \nu^{\prime} \epsilon_{a b} P_{b} \\
\Omega\left(H, P_{a}\right) H & =\left(\nu^{\prime 2}-(\xi+1)(\xi+\gamma)\right) P_{a}+\nu^{\prime}(2 \xi+1+\gamma) \epsilon_{a b} P_{b} .
\end{aligned}
$$

There are precisely two torsion-free, flat invariant connections, whose Nomizu maps are identical to those for $D \geq 3$ in equation (4.35).

In $D=1$, the torsion and curvature have the following non-zero components:

$$
\Theta(H, P)=(\nu-\xi-(1+\gamma)) P \quad \text { and } \quad \Omega(H, P) H=-(\xi+1)(\xi+\gamma) P .
$$

There are two one-parameter families of torsion-free, flat invariant connections. They have Nomizu maps

$$
\begin{aligned}
& \alpha(H, H)=(\gamma-1) H+\mu^{\prime} P \\
& \alpha(H, P)=\gamma P \quad \text { and } \\
& \alpha(P, H)=-P \\
& \alpha(H, H)=(1-\gamma) H+\mu^{\prime} P \\
& \alpha(H, P)=P \\
& \alpha(P, H)=-\gamma P \text {. }
\end{aligned}
$$

\subsubsection{Torsional galilean anti de Sitter spacetime $\left(\right.$ AdSG $\left._{\chi}\right)$}

The torsion and curvature of the connection corresponding to this Nomizu map in $D \geq 3$ are given by the following non-zero components:

$$
\Theta\left(H, P_{a}\right)=(\nu-\xi-2 \chi) P_{a} \quad \text { and } \quad \Omega\left(H, P_{a}\right) H=-\left(1+(\xi+\chi)^{2}\right) P_{a} .
$$

Therefore, we see that there are no flat invariant connections; although there is a oneparameter family of torsion-free invariant connections.

For $D=2$, we have a three-parameter family of invariant connections for which the torsion and curvature are given by the following non-zero components:

$$
\begin{aligned}
\Theta\left(H, P_{a}\right) & =(\nu-\xi-2 \chi) P_{a}+2 \nu^{\prime} \epsilon_{a b} P_{b} \\
\text { and } \quad \Omega\left(H, P_{a}\right) H & =\left(\left(v^{\prime}\right)^{2}-(\xi+\chi)^{2}-1\right) P_{a}+2 \nu^{\prime}(\xi+\chi) \epsilon_{a b} P_{b} .
\end{aligned}
$$

Again, there are no flat invariant connections, but there is a two-parameter family of torsion-free invariant connections.

Let $D=1$. We calculate the torsion and curvature to be

$$
\Theta(H, P)=(\nu-\xi-2 \chi) P \quad \text { and } \quad \Omega(H, P) H=-\left(1+(\xi+\chi)^{2}\right) P,
$$

respectively. As in higher dimensions, we thus find there to be no flat invariant connections. There is, however, a two-parameter family of torsion-free invariant connections.

\subsubsection{Spacetime $\mathrm{S} 12_{\gamma, \chi}$}

Since this spacetime is particular to $D=2$ and reductive, we need only consider the $D=2$ case of (4.11) and we may use equation (3.42) to obtain the following torsion and curvature

$$
\begin{aligned}
\Theta\left(H, P_{a}\right) & =(\nu-\xi-(1+\gamma)) P_{a}+\left(2 \nu^{\prime}+\chi\right) \epsilon_{a b} P_{b} \\
\Omega\left(H, P_{a}\right) H & =\left(\nu^{\prime}\left(\nu^{\prime}+\chi\right)-(\xi+1)(\xi+\gamma)\right) P_{a}+\left(2 \nu^{\prime} \xi+(1+\gamma) \nu^{\prime}+(1+\xi) \chi\right) \epsilon_{a b} P_{b} .
\end{aligned}
$$


For the torsion to vanish we need $\nu^{\prime}=-\chi / 2$ and $\nu-\xi=1+\gamma$. If, in addition, the curvature were to vanish we would find

$$
0=2 \nu^{\prime} \xi+(1+\gamma) \nu^{\prime}+(1+\xi) \chi=-\frac{1}{2}(\gamma-1) \chi .
$$

Hence torsion-free, flat invariant connections require either $\gamma=1$ or $\chi=0$. Both of these values lie outside the range of their corresponding parameter. From the vanishing of the $P_{a}$ term in the curvature, we see that $\chi=0$ is necessary, which agrees with the previous results: torsional galilean de Sitter spacetimes ( $\mathrm{dSG}_{\gamma}$ ) admit torsion-free, flat invariant connections, but torsional galilean anti de Sitter spacetimes $\left(\operatorname{AdSG}_{\chi}\right)$ do not (unless $\chi=0$ ).

\subsection{Nomizu maps for carrollian spacetimes}

On a carrollian spacetime, the boosts act as

$$
\lambda_{B_{a}} P_{b}=\delta_{a b} H
$$

and $H$ is invariant. This results in the following invariant Nomizu maps:

$$
\begin{aligned}
\alpha(H, H) & =0 \\
\alpha\left(P_{a}, P_{b}\right) & =\left\{\begin{array}{ll}
\zeta \delta_{a b} H & \alpha\left(H, P_{a}\right)= \begin{cases}0 & D \geq 2 \\
\delta_{a b} H+\zeta^{\prime} \epsilon_{a b} H & D=2 \\
\zeta H+\left(\nu^{\prime}+\xi^{\prime}\right) P & D=1\end{cases}
\end{array} \quad \alpha\left(P_{a}, H\right)= \begin{cases}0 & D \geq 2 \\
\xi^{\prime} H & D=1 .\end{cases} \right.
\end{aligned}
$$

\subsubsection{Carrollian spacetimes (C)}

For $D \geq 3$, the corresponding invariant connections are flat and torsion-free for all values of $\zeta$.

Letting $D=2$, we find the following non-vanishing torsion component

$$
\Theta\left(P_{a}, P_{b}\right)=2 \zeta^{\prime} \epsilon_{a b} H
$$

We, therefore, have the same torsion-free, flat invariant connections that were found in higher dimensions.

For $D=1$, the torsion and curvature are easily calculated to be

$$
\Theta(H, P)=\left(\nu^{\prime}-\xi^{\prime}\right) H \quad \Omega(H, P) P=\left(\nu^{\prime}\right)^{2} H .
$$

We thus find a one-parameter family of torsion-free, flat invariant connections, as in higher dimensions:

$$
\alpha(P, P)=\zeta H
$$

\subsection{2 (Anti) de Sitter carrollian spacetimes (dSC and AdSC)}

We will treat these two spacetimes together by introducing $\varkappa= \pm 1$. Carrollian de Sitter spacetime (dSC) corresponds to $\varkappa=1$ and carrollian anti de Sitter spacetime (AdSC) to $\varkappa=-1$. 
If $D \geq 3$, the torsion vanishes and the curvature has the following non-zero components:

$$
\Omega\left(H, P_{a}\right) P_{b}=\varkappa \delta_{a b} H \quad \text { and } \quad \Omega\left(P_{a}, P_{b}\right) P_{c}=\varkappa\left(\delta_{b c} P_{a}-\delta_{a c} P_{b}\right),
$$

which is never flat. Both of these results are independent of the Nomizu map.

If $D=2$, the non-zero components of the torsion and curvature are given by

$$
\begin{aligned}
\Theta\left(P_{a}, P_{b}\right) & =2 \zeta^{\prime} \epsilon_{a b} H, \\
\Omega\left(H, P_{a}\right) P_{b} & =\epsilon \delta_{a b} H, \text { and }, \\
\Omega\left(P_{a}, P_{b}\right) P_{c} & =\epsilon\left(\delta_{b c} P_{a}-\delta_{a c} P_{b}\right) .
\end{aligned}
$$

It is torsion-free if $\zeta^{\prime}=0$, but it is never flat.

Finally, if $D=1$, then the non-zero components of the torsion and curvature are

$$
\begin{aligned}
\Theta(H, P) & =\left(\nu^{\prime}-\xi^{\prime}\right) H \\
\Omega(H, P) P & =\left(\varkappa+\nu^{\prime 2}\right) H,
\end{aligned}
$$

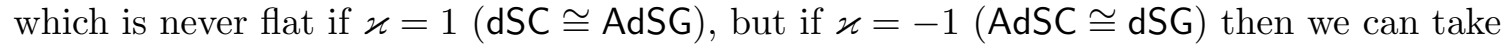
$\nu^{\prime}=\xi^{\prime}= \pm 1$, to yield two one-parameter families of torsion-free, flat connections with Nomizu maps:

$$
\begin{aligned}
& \alpha(H, P)=H \quad \alpha(H, P)=-H \\
& \alpha(P, H)=H \quad \text { and } \quad \alpha(P, H)=-H \\
& \alpha(P, P)=\zeta H+2 P \quad \alpha(P, P)=\zeta H-2 P .
\end{aligned}
$$

\subsubsection{Carrollian light cone (LC)}

As show in [6], this homogeneous spacetime does not admit any invariant connections for $D \geq 2$. For $D=1$, there is a three-parameter family of invariant connections and a unique torsion-free, flat invariant connection.

\subsection{Nomizu maps for exotic two-dimensional spacetimes}

In the bottom section of table 1 there are exotic two-dimensional reductive spacetimes with no discernible structure, and we must study their Nomizu maps separately. We can distinguish the four types of spacetime by the action of $\lambda_{B}$ on the two-dimensional space $\mathfrak{m}$ spanned by $P$ and $H$.

In the case of spacetime $\mathrm{S} 17, \lambda_{B}$ is not diagonalisable. Therefore, one needs to study the linear system defined by $\lambda_{B} \alpha=0$. Having done so, one deduces that the only invariant Nomizu map is the zero map.

For all the remaining spacetimes, $\lambda_{B}$ acts semi-simply: diagonally over $\mathbb{R}$ for spacetimes $\mathrm{S} 18$ and $\mathrm{S} 19_{\chi}$ and diagonally over $\mathbb{C}$ for spacetime $\mathrm{S} 20_{\chi}$. In spacetime $\mathrm{S} 18, \lambda_{B}$ is minus the identity on $\mathfrak{m}$, hence the identity on $\mathfrak{m}^{*}$. By parity, there are no zero eigenvalues in $\mathfrak{m}^{*} \otimes \mathfrak{m}^{*} \otimes \mathfrak{m}$, and hence the only invariant Nomizu map is the zero map.

In spacetime $\mathrm{S} 19_{\chi}, \lambda_{B}$ acts diagonally on $\mathfrak{m}$ with eigenvalues $1-\chi$ and $-1-\chi$. Letting $V_{h}$ denote the one-dimensional module of $B$ with weight $h$, we see that as a $B$-module, $\mathfrak{m} \cong V_{1-\chi} \oplus V_{-1-\chi}$, so that $\mathfrak{m}^{*} \cong V_{-1+\chi} \oplus V_{1+\chi}$. Therefore,

$$
\mathfrak{m}^{*} \otimes \mathfrak{m}^{*} \cong V_{2 \chi-2} \oplus V_{2 \chi+2} \oplus 2 V_{2 \chi} \quad \text { and } \quad \mathfrak{m}^{*} \otimes \mathfrak{m}^{*} \otimes \mathfrak{m} \cong 3 V_{\chi+1} \oplus 3 V_{\chi-1} \oplus V_{\chi+3} \oplus V_{\chi-3}
$$


Therefore, for generic $\chi>0$, there are no invariant Nomizu maps other than the zero map. But, for $\chi=1$ there are three invariants:

$$
\alpha(H, P)=\nu^{\prime} H, \quad \alpha(P, H)=\xi^{\prime} H, \quad \text { and } \quad \alpha(P, P)=\zeta^{\prime} P,
$$

and for $\chi=3$ there is one invariant:

$$
\alpha(P, P)=\zeta H
$$

In the limit $\chi \rightarrow \infty$, spacetime S19 $\chi$ tends to spacetime S18. Since there are no non-zero invariant Nomizu maps for generic $\chi$, we expect the same is true in the limit, which agrees with our previous findings.

Finally, in spacetime $\mathrm{S} 20_{\chi}, \lambda_{B}$ is semi-simple with complex eigenvalues, hence diagonalisable in the complexification $\mathfrak{m}_{\mathbb{C}}$ of $\mathfrak{m}$. If now $V_{h}$ denotes the complex one-dimensional $B$-module with weight $h$, we have that as $B$-modules

$$
\mathfrak{m}_{\mathbb{C}} \cong V_{-\chi+i} \oplus V_{-\chi-i} \quad \text { and hence } \quad \mathfrak{m}_{\mathbb{C}}^{*} \cong V_{\chi-i} \oplus V_{\chi+i}
$$

The imaginary parts of the weights of $\mathfrak{m}_{\mathbb{C}}$ and $\mathfrak{m}_{\mathbb{C}}^{*}$ are $\pm i$, so (by parity) there cannot be any real weights in $\mathfrak{m}_{\mathbb{C}}^{*} \otimes \mathfrak{m}_{\mathbb{C}}^{*} \otimes \mathfrak{m}_{\mathbb{C}}$ and, in particular, no zero weights. Had there been a zero weight in $\mathfrak{m}^{*} \otimes \mathfrak{m}^{*} \otimes \mathfrak{m}$, this would have resulted in a zero weight in $\mathfrak{m}_{\mathbb{C}}^{*} \otimes \mathfrak{m}_{\mathbb{C}}^{*} \otimes \mathfrak{m}_{\mathbb{C}}$ upon complexification. Therefore there are no zero weights in $\mathfrak{m}^{*} \otimes \mathfrak{m}^{*} \otimes \mathfrak{m}$ and hence the only invariant Nomizu map is the zero map.

\subsection{Nomizu maps for aristotelian spacetimes}

In this section, we study the geometrical properties of the aristotelian spacetimes of table 2 . They are all reductive, so there is a canonical invariant connection, and any other invariant connection is determined uniquely by its Nomizu map. The Nomizu maps $\alpha: \mathfrak{m} \times \mathfrak{m} \rightarrow \mathfrak{m}$ are only subject to equivariance under rotations and are given by (4.1). They depend only on the dimension $D$ and not on the precise aristotelian spacetime; although, of course, the precise expression for the torsion and curvature tensors does depend on the spacetime. We will calculate the torsion and curvature for each spacetime below.

\subsubsection{Static spacetime (S)}

For $D \geq 4$, the torsion and curvature of the most general invariant connection has the following non-zero components:

$$
\begin{aligned}
\Theta\left(H, P_{a}\right) & =(\nu-\xi) P_{a}, \\
\Omega\left(H, P_{a}\right) H & =\xi(\nu-\mu) P_{a}, \\
\Omega\left(H, P_{a}\right) P_{b} & =\zeta(\mu-\nu) \delta_{a b} H, \quad \text { and } \\
\Omega\left(P_{a}, P_{b}\right) P_{c} & =\zeta \xi\left(\delta_{b c} P_{a}-\delta_{a c} P_{b}\right) .
\end{aligned}
$$

There are three classes of torsion-free, flat invariant connections in addition to the canonical connection: 
1. $\zeta=0$ and $\mu=\nu=\xi \neq 0$,

2. $\nu=\xi=\zeta=0$ and $\mu \neq 0$, and

3. $\mu=\nu=\xi=0$ and $\zeta \neq 0$.

For $D=3$, the torsion and curvature have the following non-zero components:

$$
\begin{aligned}
\Theta\left(H, P_{a}\right) & =(\nu-\xi) P_{a}, \\
\Theta\left(P_{a}, P_{b}\right) & =2 \zeta^{\prime} \epsilon_{a b c} P_{c}, \\
\Omega\left(H, P_{a}\right) H & =\xi(\nu-\mu) P_{a}, \\
\Omega\left(H, P_{a}\right) P_{b} & =\zeta(\mu-\nu) \delta_{a b} H, \\
\Omega\left(P_{a}, P_{b}\right) H & =2 \xi \zeta^{\prime} \epsilon_{a b c} P_{c}, \quad \text { and } \\
\Omega\left(P_{a}, P_{b}\right) P_{c} & =\left(\zeta \xi-\zeta^{\prime 2}\right)\left(\delta_{b c} P_{a}-\delta_{a c} P_{b}\right)+2 \zeta \zeta^{\prime} \epsilon_{a b c} H .
\end{aligned}
$$

The torsion-free condition implies that $\zeta^{\prime}=0$. With this value of $\zeta^{\prime}$, the above components reduce to those in the case $D \geq 4$. We, therefore, end up with the same torsion-free, flat invariant connections.

In $D=2$, the torsion and curvature have components

$$
\begin{aligned}
\Theta\left(H, P_{a}\right) & =(\nu-\xi) P_{a}+\left(\nu^{\prime}-\xi^{\prime}\right) \epsilon_{a b} P_{b}, \\
\Theta\left(P_{a}, P_{b}\right) & =2 \zeta^{\prime} \epsilon_{a b} H, \\
\Omega\left(H, P_{a}\right) H & =\left(\xi(\nu-\mu)-\xi^{\prime} \nu^{\prime}\right) P_{a}+\left(\xi \nu^{\prime}+(\nu-\mu) \xi^{\prime}\right) \epsilon_{a b} P_{b}, \\
\Omega\left(H, P_{a}\right) P_{b} & =\left(\left(\zeta(\mu-\nu)-\zeta^{\prime} \nu^{\prime}\right) \delta_{a b}+\left(\zeta \nu^{\prime}+(\mu-\nu) \zeta^{\prime}\right) \epsilon_{a b}\right) H, \\
\Omega\left(P_{a}, P_{b}\right) H & =2\left(\xi \zeta^{\prime}-\xi^{\prime} \zeta\right) \epsilon_{a b} H, \quad \text { and } \\
\Omega\left(P_{a}, P_{b}\right) P_{c} & =\left(\zeta \xi+\zeta^{\prime} \xi^{\prime}\right)\left(\delta_{b c} P_{a}-\delta_{a c} P_{b}\right)+\left(\zeta \xi^{\prime}-\zeta^{\prime} \xi\right) \epsilon_{a b} P_{c} .
\end{aligned}
$$

Here we find a one-parameter family of torsion-free, flat invariant connections given by

$$
\alpha\left(P_{a}, P_{b}\right)=\zeta \delta_{a b} H
$$

Finally, in $D=1$, the torsion and curvature have the following non-vanishing: components

$$
\begin{aligned}
\Theta(H, P) & =\left(\nu^{\prime}-\xi^{\prime}\right) H+(\nu-\xi) P, \\
\Omega(H, P) H & =\left(\xi \nu^{\prime}-\zeta \mu^{\prime}\right) H+\left(\xi(\nu-\mu)+\mu^{\prime}\left(\xi^{\prime}-\zeta^{\prime}\right)\right) P, \quad \text { and } \\
\Omega(H, P) P & =\left(\zeta \mu+\nu^{\prime}\left(\zeta^{\prime}-\xi^{\prime}\right)\right) H+\left(\zeta \mu^{\prime}-\nu^{\prime} \xi\right) P .
\end{aligned}
$$

Imposing torsion-free and flatness conditions, the following classes of invariant connections are found

1. $\mu=\nu=\xi=\mu^{\prime}=0$, and $\nu^{\prime}=\xi^{\prime}=\zeta^{\prime}$,

2. $\nu=\xi=\zeta=0$, and $\nu^{\prime}=\xi^{\prime}=\zeta^{\prime}$,

3. $\mu^{\prime}=\nu^{\prime}=\xi^{\prime}=\zeta=0$, and $\mu=\nu=\xi$, 
4. $\nu=\xi=\zeta=0$, and $\nu^{\prime}=\xi^{\prime}$,

5. $\zeta=\zeta^{\prime}=0$, and $\nu=\xi, \nu^{\prime}=\xi^{\prime}$,

6. $\nu=\xi=\mu^{\prime}=0$, and $\nu^{\prime}=\xi^{\prime}$, and,

7. $\mu=\nu=\xi=\mu^{\prime}=\nu^{\prime}=\xi^{\prime}=0$.

Since the remaining aristotelian spacetimes, all have the same Nomizu maps as this static case, all of them will have the above torsion and curvature components as a base, with a few additional terms included due to the additional non-vanishing brackets of the specific spacetime.

\subsubsection{Torsional static spacetime (TS)}

For $D \geq 4$, the torsion and curvature are given by

$$
\begin{aligned}
\Theta\left(H, P_{a}\right) & =(\nu-\xi-1) P_{a}, \\
\Omega\left(H, P_{a}\right) H & =\xi(\nu-\mu-1) P_{a}, \\
\Omega\left(H, P_{a}\right) P_{b} & =\zeta(\mu-\nu-1) \delta_{a b} H, \quad \text { and } \\
\Omega\left(P_{a}, P_{b}\right) P_{c} & =\zeta \xi\left(\delta_{b c} P_{a}-\delta_{a c} P_{b}\right) .
\end{aligned}
$$

As in the static case, we again find three classes of torsion-free, flat invariant connection:

1. $\xi=\zeta=0$, and $\nu=1$,

2. $\mu=\xi=\nu-1$, and $\zeta=0$, and,

3. $\xi=0, \nu=1$, and $\mu=2$.

Letting $D=3$, we get the following non-vanishing torsion and curvature components:

$$
\begin{aligned}
\Theta\left(H, P_{a}\right) & =(\nu-\xi-1) P_{a}, \\
\Theta\left(P_{a}, P_{b}\right) & =2 \zeta^{\prime} \epsilon_{a b c} P_{c}, \\
\Omega\left(H, P_{a}\right) H & =\xi(\nu-\mu-1) P_{a}, \\
\Omega\left(H, P_{a}\right) P_{b} & =\zeta(\mu-\nu-1) \delta_{a b} H-\zeta^{\prime} \epsilon_{a b c} P_{c}, \\
\Omega\left(P_{a}, P_{b}\right) H & =2 \xi \zeta^{\prime} \epsilon_{a b c} P_{c}, \quad \text { and } \\
\Omega\left(P_{a}, P_{b}\right) P_{c} & =\left(\zeta \xi-\zeta^{\prime 2}\right)\left(\delta_{b c} P_{a}-\delta_{a c} P_{b}\right)+2 \zeta \zeta^{\prime} \epsilon_{a b c} H .
\end{aligned}
$$

Imposing the torsion-free condition makes $\zeta^{\prime}$ vanish such that we get the same three classes of torsion-free, flat invariant connections as in the $D \geq 4$ case.

In $D=2$, the torsion and curvature are given by

$$
\begin{aligned}
\Theta\left(H, P_{a}\right) & =(\nu-\xi-1) P_{a}+\left(\nu^{\prime}-\xi^{\prime}\right) \epsilon_{a b} P_{b}, \\
\Theta\left(P_{a}, P_{b}\right) & =2 \zeta^{\prime} \epsilon_{a b} H, \\
\Omega\left(H, P_{a}\right) H & =\left(\xi(\nu-\mu-1)-\xi^{\prime} \nu^{\prime}\right) P_{a}+\left(\xi^{\prime}(\nu-\mu-1)+\xi \nu^{\prime}\right) \epsilon_{a b} P_{b}, \\
\Omega\left(H, P_{a}\right) P_{b} & =\left(\zeta(\mu-\nu-1)-\nu^{\prime} \zeta^{\prime}\right) \delta_{a b} H+\left(\zeta^{\prime}(\mu-\nu-1)+\nu^{\prime} \zeta\right) \epsilon_{a b} H, \\
\Omega\left(P_{a}, P_{b}\right) H & =2\left(\xi \zeta^{\prime}-\xi^{\prime} \zeta\right) \epsilon_{a b} H, \quad \text { and } \\
\Omega\left(P_{a}, P_{b}\right) P_{c} & =\left(\zeta \xi+\zeta^{\prime} \xi^{\prime}\right)\left(\delta_{b c} P_{a}-\delta_{a c} P_{b}\right)+\left(\zeta \xi^{\prime}-\zeta^{\prime} \xi\right) \epsilon_{a b} P_{c} .
\end{aligned}
$$


Here we find a unique torsion-free, flat invariant connection with

$$
\alpha(H, H)=2 H \quad \alpha\left(H, P_{a}\right)=2 P_{a} \quad \text { and } \quad \alpha\left(P_{a}, H\right)=P_{a} .
$$

Finally, let $D=1$. The components of the torsion and curvature are

$$
\begin{aligned}
\Theta(H, P) & =\left(\nu^{\prime}-\xi^{\prime}\right) H+(\nu-\xi-1) P, \\
\Omega(H, P) H & =\left(\xi \nu^{\prime}-\zeta \mu^{\prime}-\xi^{\prime}\right) H+\left(\xi(\nu-\mu-1)+\mu^{\prime}\left(\xi^{\prime}-\zeta^{\prime}\right)\right) P, \text { and } \\
\Omega(H, P) P & =\left(\zeta(\mu-\nu)+\nu^{\prime}\left(\zeta^{\prime}-\xi^{\prime}\right)\right) H+\left(\zeta \mu^{\prime}-\nu^{\prime} \xi-\zeta^{\prime}\right) P .
\end{aligned}
$$

We find the following classes of torsion-free, flat invariant connections

1. $\xi=\zeta=\nu^{\prime}=\xi^{\prime}=\zeta^{\prime}=0$, and $\nu=1$,

2. $\xi=0, \mu=\nu=1$, and $\nu^{\prime}=\xi^{\prime}=\zeta^{\prime}=\mu^{\prime} \zeta$,

3. $\zeta=0, \mu=\xi=\nu-1$, and $\mu^{\prime}=\nu^{\prime}=\xi^{\prime}=\zeta^{\prime}=0$, and,

4. $\mu^{\prime}=0, \mu=\xi=1, \nu=2$, and $\nu^{\prime}=\xi^{\prime}=-\zeta^{\prime}=\sqrt{\frac{-\zeta}{2}}$, for when $\zeta \leq 0$.

\subsubsection{Aristotelian spacetime $\mathrm{A23}_{\varepsilon}$}

In $D \geq 4$, the torsion and curvature are given by

$$
\begin{aligned}
\Theta\left(H, P_{a}\right) & =(\nu-\xi) P_{a}, \\
\Omega\left(H, P_{a}\right) H & =\xi(\nu-\mu) P_{a}, \\
\Omega\left(H, P_{a}\right) P_{b} & =\zeta(\mu-\nu) \delta_{a b} H, \quad \text { and } \\
\Omega\left(P_{a}, P_{b}\right) P_{c} & =(\zeta \xi+\varepsilon)\left(\delta_{b c} P_{a}-\delta_{a c} P_{b}\right) .
\end{aligned}
$$

Imposing flatness, we find that this requires $\varepsilon$ to vanish; therefore, since $\varepsilon= \pm 1$, we find no torsion-free, flat invariant connections.

Let $D=3$. The non-vanishing torsion and curvature components are

$$
\begin{aligned}
\Theta\left(H, P_{a}\right) & =(\nu-\xi) P_{a}, \\
\Theta\left(P_{a}, P_{b}\right) & =2 \zeta^{\prime} \epsilon_{a b c} P_{c} \\
\Omega\left(H, P_{a}\right) H & =\xi(\nu-\mu) P_{a}, \\
\Omega\left(H, P_{a}\right) P_{b} & =\zeta(\mu-\nu) \delta_{a b} H, \\
\Omega\left(P_{a}, P_{b}\right) H & =2 \xi \zeta^{\prime} \epsilon_{a b c} P_{c}, \quad \text { and } \\
\Omega\left(P_{a}, P_{b}\right) P_{c} & =\left(\zeta \xi+\varepsilon-\zeta^{\prime 2}\right)\left(\delta_{b c} P_{a}-\delta_{a c} P_{b}\right)+2 \zeta \zeta^{\prime} \epsilon_{a b c} H .
\end{aligned}
$$

As in the static and torsional static cases, imposing the torsion-free condition sets $\zeta^{\prime}=0$. This means we get the same torsion-free, flat invariant connections in this case as in $D \geq 4$. Therefore, there are no torsion-free, flat invariant connections in this dimension. 
In $D=2$, the torsion and curvature become

$$
\begin{aligned}
\Theta\left(H, P_{a}\right) & =(\nu-\xi) P_{a}+\left(\nu^{\prime}-\xi^{\prime}\right) \epsilon_{a b} P_{b}, \\
\Theta\left(P_{a}, P_{b}\right) & =\left(2 \zeta^{\prime}\right) \epsilon_{a b} H, \\
\Omega\left(H, P_{a}\right) H & =\left(\xi(\nu-\mu)-\xi^{\prime} \nu^{\prime}\right) P_{a}+\left(\xi^{\prime}(\nu-\mu)+\xi \nu^{\prime}\right) \epsilon_{a b} P_{b}, \\
\Omega\left(H, P_{a}\right) P_{b} & =\left(\zeta(\mu-\nu)-\nu^{\prime} \zeta^{\prime}\right) \delta_{a b} H+\left(\zeta^{\prime}(\mu-\nu)+\nu^{\prime} \zeta\right) \epsilon_{a b} H, \\
\Omega\left(P_{a}, P_{b}\right) H & =2\left(\xi \zeta^{\prime}-\xi^{\prime} \zeta\right) \epsilon_{a b} H, \quad \text { and } \\
\Omega\left(P_{a}, P_{b}\right) P_{c} & =\left(\zeta \xi+\zeta^{\prime} \xi^{\prime}+\varepsilon\right)\left(\delta_{b c} P_{a}-\delta_{a c} P_{b}\right)+\left(\zeta \xi^{\prime}-\zeta^{\prime} \xi\right) \epsilon_{a b} P_{c} .
\end{aligned}
$$

Once again, we find no torsion-free, flat invariant connections for this spacetime.

\subsubsection{Aristotelian spacetime A24}

The non-vanishing torsion and curvature components are

$$
\begin{aligned}
\Theta\left(H, P_{a}\right) & =(\nu-\xi) P_{a}+\left(\nu^{\prime}-\xi^{\prime}\right) \epsilon_{a b} P_{b}, \\
\Theta\left(P_{a}, P_{b}\right) & =\left(2 \zeta^{\prime}-1\right) \epsilon_{a b} H, \\
\Omega\left(H, P_{a}\right) H & =\left(\xi(\nu-\mu)-\xi^{\prime} \nu^{\prime}\right) P_{a}+\left(\xi^{\prime}(\nu-\mu)+\xi \nu^{\prime}\right) \epsilon_{a b} P_{b}, \\
\Omega\left(H, P_{a}\right) P_{b} & =\left(\zeta(\mu-\nu)-\nu^{\prime} \zeta^{\prime}\right) \delta_{a b} H+\left(\zeta^{\prime}(\mu-\nu)+\nu^{\prime} \zeta\right) \epsilon_{a b} H, \\
\Omega\left(P_{a}, P_{b}\right) H & =\left(2\left(\xi \zeta^{\prime}-\xi^{\prime} \zeta\right)-\mu\right) \epsilon_{a b} H, \quad \text { and } \\
\Omega\left(P_{a}, P_{b}\right) P_{c} & =\left(\zeta \xi+\zeta^{\prime} \xi^{\prime}+\nu^{\prime}\right)\left(\delta_{b c} P_{a}-\delta_{a c} P_{b}\right)+\left(\zeta \xi^{\prime}-\zeta^{\prime} \xi-\nu\right) \epsilon_{a b} P_{c} .
\end{aligned}
$$

We find a unique torsion-free, flat invariant connection. The corresponding non-vanishing Nomizu maps are

$$
\alpha\left(P_{a}, P_{b}\right)=\frac{9}{4} \delta_{a b} H+\frac{1}{2} \epsilon_{a b} H
$$

\section{Pseudo-riemannian spacetimes and their limits}

Let us introduce parameters $\varkappa=0, \pm 1, \varsigma=0, \pm 1$, and $c$, and consider the following Lie brackets in addition to (2.2):

$$
\begin{array}{rlrl}
{[H, \boldsymbol{B}]} & =\varsigma \boldsymbol{P}, \\
{[\boldsymbol{B}, \boldsymbol{B}]} & =-\frac{\varsigma}{c^{2}} \boldsymbol{J}, & {[H, \boldsymbol{P}]} & =-\varkappa \boldsymbol{B},
\end{array} \quad[\boldsymbol{B}, \boldsymbol{P}]=\frac{1}{c^{2}} H,
$$

The parameter $\varsigma$ corresponds to the signature: $\varsigma=1$ for riemannian, $\varsigma=-1$ for lorentzian and $\varsigma=0$ for carrollian. The parameter $\varkappa$ corresponds to the curvature, so $\varkappa=1,0,-1$ for positive, zero and negative curvature, respectively. ${ }^{9}$ The limit $c \rightarrow \infty$ corresponds

\footnotetext{
${ }^{9}$ This has to be taken with a grain of salt. Indeed, it follows from table 4 that the correspondence between $\varkappa$ and the sign of the curvature is a little fictitious in the galilean setting, at least: if we interpret them as limits of lorentzian spacetimes, then dSG has "positive" curvature and AdSG has "negative" curvature, but if we interpret them as limits of riemannian spaces, then it's the other way around. This means that these spacetimes are characterised by the product $\varsigma \varkappa$ (for $\mathrm{G}$ the sign is irrelevant). Concerning the carrollian spacetimes it is useful to realise that subalgebra spanned by $\boldsymbol{J}$ and $\boldsymbol{P}$ is isomorphic to $\mathfrak{s o}(D+1)$ and $\mathfrak{s o}(D, 1)$ for dSC and AdSC, respectively (see also section 10.2). Compared to the limits of section 5 in [6] we change $\tau^{2} \eta_{00} \rightarrow \varsigma$ and $\kappa^{2} \eta_{\text {珀 }} \rightarrow \varkappa$.
} 


\begin{tabular}{|c|c|c|c|}
\hline$\varsigma$ & $\varkappa$ & $c^{-1}$ & Spacetime \\
\hline-1 & 0 & 1 & Minkowski (M) \\
\hline-1 & 1 & 1 & de Sitter (dS) \\
\hline-1 & -1 & 1 & anti de Sitter (AdS) \\
\hline 1 & 0 & 1 & euclidean $(\mathbb{E})$ \\
\hline 1 & 1 & 1 & sphere $(\mathbb{S})$ \\
\hline 1 & -1 & 1 & hyperbolic $(\mathbb{H})$ \\
\hline$\mp 1$ & 0 & 0 & galilean (G) \\
\hline$\mp 1$ & \pm 1 & 0 & galilean de Sitter (dSG) \\
\hline$\mp 1$ & $\mp 1$ & 0 & galilean anti de Sitter (AdSG) \\
\hline 0 & 0 & 1 & carrollian (C) \\
\hline 0 & 1 & 1 & carrollian de Sitter (dSC) \\
\hline 0 & -1 & 1 & carrollian anti de Sitter (AdSC) \\
\hline
\end{tabular}

Table 4. Symmetric spacetimes.

to the non-relativistic limit. In the computations below we will work with unspecified values of $\varsigma, \varkappa, c$ and only at the end will we set them to appropriate values to recover the results for particular spacetimes. Some of the expressions will have (removable) singularities whenever $\varsigma$ or $\varkappa$ vanish, so will have to think of those cases as limits: the ultra-relativistic limit $\varsigma \rightarrow 0$ and the flat limit $\varkappa \rightarrow 0$. Table 4 shows the spacetimes associated to different values of these parameters. They can be characterised as those homogeneous kinematical spacetimes which are symmetric, so the canonical invariant connection is torsion-free. The table divides into four sections separated by horizontal rules corresponding, from top to bottom, to lorentzian, euclidean, galilean and carrollian symmetric spacetimes.

\subsection{Invariant structures}

We will determine the form of the invariant tensors of small rank. If $\mathfrak{k}=\mathfrak{h} \oplus \mathfrak{m}$ is a reductive split then, as explained in section 3.7, invariant tensor fields on a simply-connected homogeneous space $M=\mathcal{K} / \mathcal{H}$ are in bijective correspondence with $\mathcal{H}$-invariant tensors on $\mathfrak{m}$, and since $\mathcal{H}$ is connected, these are in bijective correspondence with $\mathfrak{h}$-invariant tensors on $\mathfrak{m}$.

The action of $\mathfrak{h}$ on $\mathfrak{m}$ is the linear isotropy representation, which is the restriction to $\mathfrak{h}$ of the adjoint action:

$$
\begin{aligned}
& J_{a b} \cdot H=0 \\
& J_{a b} \cdot P_{c}=\delta_{b c} P_{a}-\delta_{a c} P_{b} \\
& B_{a} \cdot H=-\varsigma P_{a} \\
& B_{a} \cdot P_{b}=\frac{1}{c^{2}} \delta_{a b} H .
\end{aligned}
$$

With respect to the canonical dual basis $\eta, \pi_{a}$ for $\mathfrak{m}^{*}$, the dual linear isotropy representation is the restriction of the coadjoint action:

$$
\begin{aligned}
J_{a b} \cdot \eta & =0 \\
J_{a b} \cdot \pi^{c} & =-\delta_{a}^{c} \pi_{b}+\delta_{b}^{c} \pi_{a}
\end{aligned} \quad \text { and } \quad \begin{aligned}
B_{a} \cdot \eta & =-\frac{1}{c^{2}} \pi_{a} \\
B_{a} \cdot \pi^{b} & =\varsigma \delta_{a}^{b} \eta .
\end{aligned}
$$

It follows that $H$ is invariant in the $\sigma \rightarrow 0$ limit, whereas $\eta$ is invariant in the $c \rightarrow \infty$ limit. 
Concerning the rotationally invariant tensors of second rank, let us observe that

$$
\alpha_{1} H^{2}+\beta_{1} \boldsymbol{P}^{2} \quad \text { is invariant } \Longleftrightarrow \sigma \alpha_{1}=\frac{1}{c^{2}} \beta_{1}
$$

and

$$
\alpha_{2} \eta^{2}+\beta_{2} \pi^{2} \quad \text { is invariant } \Longleftrightarrow \frac{1}{c^{2}} \alpha_{2}=\sigma \beta_{2} .
$$

It is interesting to note that the sign $\varkappa$ of the curvature has played no rôle thus far.

We shall now specialise to the different classes of spacetimes and determine whether and how the structures are induced in the limit.

\subsubsection{Lorentzian and riemannian case}

It is clear that for the (pseudo-)riemannian case, where $\varsigma \neq 0 \neq \frac{1}{c^{2}}$, only the metric and its co-metric are invariant. Keeping in mind that we wish the limit in which the parameters $\varsigma$ and $c$ tend to zero to exist, we set $\alpha_{1}=\frac{1}{c^{2}}$ and $\beta_{1}=\varsigma$ and similarly for the co-metric, which leads to the invariants

$$
\frac{1}{c^{2}} H^{2}+\varsigma \boldsymbol{P}^{2} \quad \text { and } \quad \varsigma \eta^{2}+\frac{1}{c^{2}} \boldsymbol{\pi}^{2} .
$$

For negative (positive) $\varsigma$ this is the invariant lorentzian (riemannian) structure. The metric and the co-metric are not per se the inverse of each other, although using definite values for the limiting parameters they can be made to be.

\subsubsection{Non- and ultra-relativistic limits}

Let us now investigate the limits. Taking the non-relativistic limit $(c \rightarrow \infty)$ of the metrics leads to the invariants

$$
\varsigma \boldsymbol{P}^{2} \quad \text { and } \quad \varsigma \eta^{2}
$$

which can be interpreted as the invariants that properly arise from the lorentzian structure. However, as (5.3) shows also $\eta$ itself is an invariant in this limit. This does not follow from the contractions, but can be anticipated from the metrics. We could now take the ultra-relativistic limit $(\varsigma \rightarrow 0)$ of $(5.8)$ leading to no invariant tensor. Of course, this spacetime has the invariants $H, \boldsymbol{P}^{2}, \eta, \boldsymbol{\pi}^{2}$, but none of these arise from the limit of the original lorentzian and riemannian metrics. For the ultra-relativistic limit, we may apply the same logic.

Concluding, we have the galilean structure $\eta, \varsigma \boldsymbol{P}^{2}$ and the carrollian structure $H, \frac{1}{c^{2}} \boldsymbol{\pi}^{2}$, where we have left the contraction parameters for the invariants that arise from a limit.

\subsection{Action of the boosts}

The actions of the boosts for all the lorentzian, riemannian, galilean, and reductive carrollian spacetimes were determined in section 3.4, where we arrived at equation (3.38) for the orbit of $\left(t_{0}, \boldsymbol{x}_{0}\right)$ under the one-parameter family of boosts generated by $\boldsymbol{w} \cdot \boldsymbol{B}$, which we rewrite here as follows:

$$
\begin{aligned}
& t(s)=t_{0} \cosh (s z)+\frac{1}{c^{2}} \frac{\sinh (s z)}{z} \boldsymbol{x}_{0} \cdot \boldsymbol{w} \\
& \boldsymbol{x}(s)=\boldsymbol{x}_{0}^{\perp}-\varsigma t_{0} \frac{\sinh (s z)}{z} \boldsymbol{w}+\cosh (s z) \frac{\left(\boldsymbol{x}_{0} \cdot \boldsymbol{w}\right)}{w^{2}} \boldsymbol{w}
\end{aligned}
$$


where $\boldsymbol{x}_{0}^{\perp}:=\boldsymbol{x}_{0}-\frac{\boldsymbol{x}_{0} \cdot \boldsymbol{w}}{w^{2}} \boldsymbol{w}$ and $z^{2}:=-\frac{1}{c^{2}} \varsigma w^{2}$. Notice that the orbits of $\left(0, \boldsymbol{x}_{0}\right)$ with $\boldsymbol{x}_{0} \cdot \boldsymbol{w}=0$ are point-like. To understand the nature of the other (generic) orbits, we choose values for the parameters. Notice that in our coset parametrisation the boosts do not depend on $\varkappa$, but only on $\varsigma$ and $c$. Therefore, we shall be able to treat each class of spacetime uniformly.

\subsubsection{Lorentzian boosts}

Here we take $\varsigma=-1$ and keep $c^{-1}$ non-zero. Then $z^{2}=\frac{w^{2}}{c^{2}}$, so $z=\left|\frac{\boldsymbol{w}}{c}\right|$, and the orbits of the boosts are

$$
\begin{aligned}
& t(s)=t_{0} \cosh \left(s\left|\frac{\boldsymbol{w}}{c}\right|\right)+\frac{1}{c^{2}} \frac{\sinh \left(s\left|\frac{\boldsymbol{w}}{c}\right|\right)}{\left|\frac{\boldsymbol{w}}{c}\right|} \boldsymbol{x}_{0} \cdot \boldsymbol{w} \\
& \boldsymbol{x}(s)=\boldsymbol{x}_{0}^{\perp}+t_{0} \frac{\sinh \left(s\left|\frac{\boldsymbol{w}}{c}\right|\right)}{\left|\frac{\boldsymbol{w}}{c}\right|} \boldsymbol{w}+\cosh \left(s\left|\frac{\boldsymbol{w}}{c}\right|\right) \frac{\left(\boldsymbol{x}_{0} \cdot \boldsymbol{w}\right)}{w^{2}} \boldsymbol{w} .
\end{aligned}
$$

Let $\boldsymbol{x}=\boldsymbol{x}^{\perp}+y \boldsymbol{w}$, where $\boldsymbol{x}^{\perp} \cdot \boldsymbol{w}=0$. Then $\boldsymbol{x}^{\perp}(s)=\boldsymbol{x}_{0}^{\perp}$ for all $s$ and the orbit takes place in the $(t, y)$ plane. Letting $|\boldsymbol{w}|=1$ and $c=1$, we find

$$
t(s)=t_{0} \cosh (s)+\sinh (s) y_{0} \quad \text { and } \quad y(s)=t_{0} \sinh (s)+\cosh (s) y_{0},
$$

which is either a point (if $t_{0}=y_{0}=0$ ), a straight line (if $t_{0}= \pm y_{0} \neq 0$ ), or a hyperbola (otherwise). The nature of the orbits in the exponential coordinates is clear, but only in the case of Minkowski spacetime do the exponential coordinates provide a global chart and hence only in that case can we deduce from this calculation that the generic orbits are not compact. For (anti) de Sitter spacetime, we must argue in a different way.

Let $\overline{d S}$ denote the quotient of $d S$ which embeds as a quadric hypersurface in Minkowski spacetime. The covering map $d S \rightarrow \overline{d S}$ relates the orbits of the boosts on $d S$ and in the quotient $\overline{\mathrm{dS}}$ and since continuous maps send compact sets to compact sets, it is enough to show the non-compactness of the orbits in $\overline{\mathrm{dS}}$. The embedding $\overline{\mathrm{dS}} \subset \mathbb{R}^{D+1,1}$ is given by the quadric

$$
x_{1}^{2}+\cdots+x_{D}^{2}+x_{D+1}^{2}-x_{D+2}^{2}=R^{2},
$$

which is acted on transitively by $\mathrm{SO}(D+1,1)$. The stabiliser Lie algebra of the point $(0, \cdots, 0, R, 0)$ is spanned by the $\mathfrak{s o}(D+1,1)$ generators $J_{a b}$ and $J_{a, D+2}$, so that $B_{a}=$ $J_{a, D+2}$, which is a boost in $\mathbb{R}^{D+1,1}$. We have just shown that boosts in Minkowski spacetime have non-compact orbits; therefore, this is the case in $\overline{\mathrm{dS}}$ and hence also in dS.

Similarly, let $\overline{\mathrm{AdS}}$ denote the quotient of AdS which embeds in $\mathbb{R}^{D, 2}$ as the quadric

$$
x_{1}^{2}+\cdots+x_{D}^{2}-x_{D+1}^{2}-x_{D+2}^{2}=-R^{2} .
$$

The Lie algebra $\mathfrak{s o}(D, 2)$ acts transitively on this quadric and the stabiliser Lie algebra at the point $(0, \cdots, 0,0, R)$ is spanned by the $\mathfrak{s o}(D, 2)$ generators $J_{a b}$ and $J_{a, D+1}$, so that $B_{a}=J_{a, D+1}$ which is a "boost" in $\mathbb{R}^{D, 2}$. The calculation of the orbit, in this case, is formally identical to the one for Minkowski spacetime (in fact, it takes place in the lorentzian plane with coordinates $\left.\left(x_{a}, x_{D+2}\right)\right)$ and we see that they are non-compact, so the same holds in $\overline{\mathrm{AdS}}$ and thus also in AdS. 


\subsubsection{Euclidean "boosts"}

Here we take $\varsigma=1$ and keep $c^{-1}$ non-zero. Then $z^{2}=-\frac{w^{2}}{c^{2}}$, so $z=i\left|\frac{\boldsymbol{w}}{c}\right|$, and the orbits of the boosts are

$$
\begin{aligned}
& t(s)=t_{0} \cos \left(s\left|\frac{\boldsymbol{w}}{c}\right|\right)+\frac{1}{c^{2}} \frac{\sin \left(s\left|\frac{\boldsymbol{w}}{c}\right|\right)}{\left|\frac{\boldsymbol{w}}{c}\right|} \boldsymbol{x}_{0} \cdot \boldsymbol{w} \\
& \boldsymbol{x}(s)=\boldsymbol{x}_{0}^{\perp}-t_{0} \frac{\sin \left(s\left|\frac{\boldsymbol{w}}{c}\right|\right)}{\left|\frac{\boldsymbol{w}}{c}\right|} \boldsymbol{w}+\cos \left(s\left|\frac{\boldsymbol{w}}{c}\right|\right) \frac{\left(\boldsymbol{x}_{0} \cdot \boldsymbol{w}\right)}{w^{2}} \boldsymbol{w} .
\end{aligned}
$$

As before, letting $\boldsymbol{x}=\boldsymbol{x}^{\perp}+y \boldsymbol{w}$, and choosing $|\boldsymbol{w}|=1$ and $c=1$, we find that the orbit is such that $\boldsymbol{x}^{\perp}$ is constant and $(t, y)$ evolve as

$$
t(s)=t_{0} \cos (s)+\sin (s) y_{0} \quad \text { and } \quad y(s)=-t_{0} \sin (s)+\cos (s) y_{0},
$$

which is either a point (if $t_{0}=y_{0}=0$ ) or a circle (otherwise) and in any case compact. This suffices for $\mathbb{E}$ and $\mathbb{H}$ since the exponential coordinates give a global chart. For $\mathbb{S}$ it is clear that the boosts act with compact orbits because the kinematical Lie group $\mathrm{SO}(D+2)$ is itself compact, therefore, so are the one-parameter subgroups.

\subsubsection{Galilean boosts}

Here we take the limit $c \rightarrow \infty$ and, for definiteness, $\varsigma=-1$. The orbits of the boosts are then the limit $c \rightarrow \infty$ of equation (5.10):

$$
\begin{aligned}
t(s) & =t_{0} \\
\boldsymbol{x}(s) & =\boldsymbol{x}_{0}+s t_{0} \boldsymbol{w} .
\end{aligned}
$$

Here the orbits of $\left(0, \boldsymbol{x}_{0}\right)$ are point-like. The generic orbit $\left(t_{0} \neq 0\right)$ is not periodic and hence not compact. This suffices for $G$ and dSG, since the exponential coordinates define a global chart. For AdSG we need to argue differently and this is done in section 5.10.

\subsubsection{Carrollian boosts}

Here we keep $c^{-1}$ non-zero, but take the limit $\varsigma \rightarrow 0$ in equation (3.38):

$$
\begin{aligned}
t(s) & =t_{0}+s \frac{1}{c^{2}} \boldsymbol{x}_{0} \cdot \boldsymbol{w} \\
\boldsymbol{x}(s) & =\boldsymbol{x}_{0} .
\end{aligned}
$$

Here the orbits $\left(t_{0}, \boldsymbol{x}_{0}\right)$ with $\boldsymbol{x}_{0} \cdot \boldsymbol{w}=0$ are point-like, but the other orbits are not periodic, hence not compact. This settles it for AdSC, since the exponential coordinates give a global chart. For the other carrollian spacetimes we can argue in a different way.

As shown in [34], a carrollian spacetime admits an embedding as a null hypersurface in a lorentzian spacetime. For the homogeneous examples in this paper, this was done in [6] following the embeddings of the carrollian spacetimes C and LC as null hypersurfaces of Minkowski spacetime described already in [34].

As explained in section 3.4, for dSC it is enough to work with the discrete quotient $\overline{\mathrm{dSC}}$, which embeds as a null hypersurface in the hyperboloid model $\overline{\mathrm{dS}}$ of de Sitter spacetime, 
which itself is a quadric hypersurface in Minkowski spacetime. In [6] we showed that the boosts in $\overline{\mathrm{dSC}}$ can be interpreted as null rotations in the (higher-dimensional) pseudoorthogonal Lie group and the orbits of null rotations are never compact. This is done in detail in section 7.1 for LC.

\subsection{Fundamental vector fields}

The fundamental vector fields for rotations and boosts are linear in exponential coordinates and given by equations (3.26) and (3.39), respectively. To determine the fundamental vector fields for the translations we must work harder.

Now let $A=t H+\boldsymbol{x} \cdot \boldsymbol{P}$. Then we have that

$$
\begin{aligned}
& \operatorname{ad}_{A} H=\varkappa \boldsymbol{x} \cdot \boldsymbol{B} \\
& \operatorname{ad}_{A} B_{a}=\varsigma t P_{a}-\frac{1}{c^{2}} x_{a} H \\
& \operatorname{ad}_{A} P_{a}=\frac{\varkappa}{c^{2}} J_{a b} x^{b}-\varkappa t B_{a} \\
& \text { and } \\
& \operatorname{ad}_{A}^{2} H=\varkappa s t \boldsymbol{x} \cdot \boldsymbol{P}-\frac{\varkappa}{c^{2}} x^{2} H \\
& \operatorname{ad}_{A} J_{a b}=x_{a} P_{b}-x_{b} P_{a} \\
& \operatorname{ad}_{A}^{2} B_{a}=\frac{\varkappa}{c^{2}} \varsigma t x^{b} J_{a b}-\varkappa \varsigma t^{2} B_{a}-\frac{\varkappa}{c^{2}} x_{a} \boldsymbol{x} \cdot \boldsymbol{B} \\
& \operatorname{ad}_{A}^{2} P_{a}=-\varkappa\left(\frac{1}{c^{2}} x^{2}+\varsigma t^{2}\right) P_{a}+\frac{\varkappa}{c^{2}} x_{a} x \cdot P+\frac{\varkappa}{c^{2}} t x_{a} H \\
& \operatorname{ad}_{A}^{2} J_{a b}=-\varkappa t\left(x_{a} B_{b}-x_{b} B_{a}\right)+\frac{\varkappa}{c^{2}} x^{c}\left(x_{a} J_{b c}-x_{b} J_{a c}\right),
\end{aligned}
$$

so that in general we have

$$
\operatorname{ad}_{A}^{3}=-\varkappa\left(\frac{1}{c^{2}} x^{2}+\varsigma t^{2}\right) \operatorname{ad}_{A}
$$

Letting $x_{ \pm}$denote the two complex square roots of $-\varkappa\left(\frac{1}{c^{2}} x^{2}+\varsigma t^{2}\right)$, with $x_{-}=-x_{+}$, we can rewrite this equation as $\operatorname{ad}_{A}^{3}=x_{+}^{2} \operatorname{ad}_{A}$.

Now, if $f(z)$ is analytic in $z$ and admits a power series expansion $f(z)=$ $\sum_{n=0}^{\infty} c_{n} z^{n}$, then

$$
f\left(\operatorname{ad}_{A}\right)=f(0)+\frac{1}{x_{+}} \sum_{k=0}^{\infty} c_{2 k+1} x_{+}^{2 k+1} \operatorname{ad}_{A}+\frac{1}{x_{+}^{2}} \sum_{k=1}^{\infty} c_{2 k} x_{+}^{2 k} \operatorname{ad}_{A}^{2} .
$$

Observing that

$$
\sum_{k=0}^{\infty} c_{2 k+1} x_{+}^{2 k+1}=\frac{1}{2}\left(f\left(x_{+}\right)-f\left(x_{-}\right)\right) \quad \text { and } \quad \sum_{k=1}^{\infty} c_{2 k} x_{+}^{2 k}=\frac{1}{2}\left(f\left(x_{+}\right)+f\left(x_{-}\right)-2 f(0)\right),
$$

we arrive finally at

$$
f\left(\operatorname{ad}_{A}\right)=f(0)+\frac{1}{2 x_{+}}\left(f\left(x_{+}\right)-f\left(x_{-}\right)\right) \operatorname{ad}_{A}+\frac{1}{2 x_{+}^{2}}\left(f\left(x_{+}\right)+f\left(x_{-}\right)-2 f(0)\right) \operatorname{ad}_{A}^{2} .
$$

Introducing the shorthand notation:

$$
f^{+}:=\frac{1}{2}\left(f\left(x_{+}\right)+f\left(x_{-}\right)\right) \quad \text { and } \quad f^{-}:=\frac{1}{2 x_{+}}\left(f\left(x_{+}\right)-f\left(x_{-}\right)\right),
$$

equation (5.22) becomes

$$
f\left(\operatorname{ad}_{A}\right)=f(0)+f^{-} \operatorname{ad}_{A}+\frac{1}{x_{+}^{2}}\left(f^{+}-f(0)\right) \operatorname{ad}_{A}^{2} .
$$


It follows from the above equation and equation (5.18), that for $f(z)$ analytic in $z$,

$$
\begin{aligned}
f\left(\operatorname{ad}_{A}\right) H= & f(0) H+f^{-} \varkappa \boldsymbol{x} \cdot \boldsymbol{B} \\
& +\frac{1}{x_{+}^{2}}\left(f^{+}-f(0)\right)\left(\varkappa \varsigma t \boldsymbol{x} \cdot \boldsymbol{P}-\frac{\varkappa}{c^{2}} x^{2} H\right) \\
f\left(\operatorname{ad}_{A}\right) B_{a}= & f(0) B_{a}+f^{-}\left(\varsigma t P_{a}-\frac{1}{c^{2}} x_{a} H\right) \\
& +\frac{1}{x_{+}^{2}}\left(f^{+}-f(0)\right)\left(-\varkappa \varsigma t^{2} B_{a}-\frac{\varkappa}{c^{2}} x_{a} \boldsymbol{x} \cdot \boldsymbol{B}+\frac{\varkappa}{c^{2}} \varsigma t J_{a b} x^{b}\right) \\
f\left(\operatorname{ad}_{A}\right) P_{a}= & f^{+} P_{a}+f^{-}\left(-\varkappa t B_{a}+\frac{\varkappa}{c^{2}} J_{a b} x^{b}\right)+\frac{1}{x_{+}^{2}}\left(f^{+}-f(0)\right) \frac{\varkappa}{c^{2}} x_{a}(t H+\boldsymbol{x} \cdot \boldsymbol{P}) \\
f\left(\operatorname{ad}_{A}\right) J_{a b}= & f(0) J_{a b}+f^{-}\left(x_{a} P_{b}-x_{b} P_{a}\right) \\
& +\frac{1}{x_{+}^{2}}\left(f^{+}-f(0)\right) \varkappa\left(-t\left(x_{a} B_{b}-x_{b} B_{a}\right)+\frac{1}{c^{2}} x^{c}\left(x_{a} J_{b c}-x_{b} J_{a c}\right)\right) .
\end{aligned}
$$

Let us calculate $\xi_{H}=\tau \frac{\partial}{\partial t}+y^{a} \frac{\partial}{\partial x^{a}}$, where by equation (3.16)

$$
\tau H+\boldsymbol{y} \cdot \boldsymbol{P}=G\left(\operatorname{ad}_{A}\right) H-F\left(\operatorname{ad}_{A}\right) \boldsymbol{\beta} \cdot \boldsymbol{B},
$$

for some $\boldsymbol{\beta}$. From equation (5.25), we have

$$
\begin{aligned}
\tau H+\boldsymbol{y} \cdot \boldsymbol{P}= & H+G^{-} \varkappa \boldsymbol{x} \cdot \boldsymbol{B}+\frac{1}{x_{+}^{2}}\left(G^{+}-1\right)\left(\varkappa s t \boldsymbol{x} \cdot \boldsymbol{P}-\frac{\varkappa}{c^{2}} x^{2} H\right) \\
& -\left(\boldsymbol{\beta} \cdot \boldsymbol{B}+F^{-}\left(\varsigma t \boldsymbol{\beta} \cdot \boldsymbol{P}-\frac{1}{c^{2}} \boldsymbol{x} \cdot \boldsymbol{\beta} H\right)\right. \\
& \left.+\frac{1}{x_{+}^{2}}\left(F^{+}-1\right)\left(-\varkappa s t^{2} \boldsymbol{\beta} \cdot \boldsymbol{B}-\frac{\varkappa}{c^{2}} \boldsymbol{x} \cdot \boldsymbol{\beta} \boldsymbol{x} \cdot \boldsymbol{B}+\frac{\varkappa}{c^{2}} \varsigma t J_{a b} \beta^{a} x^{b}\right)\right) .
\end{aligned}
$$

By $\mathfrak{s o}(D)$-covariance, $\boldsymbol{\beta}$ has to be proportional to $\boldsymbol{x}$, since that is the only other vector appearing in the $\boldsymbol{B}$ terms, which means that the $J_{a b}$ term above vanishes. This leaves terms in $\boldsymbol{B}, H$, and $\boldsymbol{P}$, which allow us to solve for $\boldsymbol{\beta}, \tau$, and $\boldsymbol{y}$, respectively. The $\boldsymbol{B}$ terms cancel if and only if

$$
\boldsymbol{\beta}=\frac{G^{-}}{F^{+}} \varkappa \boldsymbol{x},
$$

which we can reinsert into the equation to solve for $\tau$ and $\boldsymbol{y}$. Doing so we find

$$
\tau=1-\left(\frac{x_{+} \operatorname{coth} x_{+}-1}{x_{+}^{2}}\right) \frac{\varkappa}{c^{2}} x^{2} \quad \text { and } \quad y^{a}=\left(\frac{x_{+} \operatorname{coth} x_{+}-1}{x_{+}^{2}}\right) \varkappa \varsigma t x^{a},
$$

so that

$$
\xi_{H}=\frac{\partial}{\partial t}+\left(\frac{x_{+} \operatorname{coth} x_{+}-1}{x_{+}^{2}}\right) \varkappa\left(\varsigma t x^{a} \frac{\partial}{\partial x^{a}}-\frac{1}{c^{2}} x^{2} \frac{\partial}{\partial t}\right) .
$$

To calculate $\xi_{\boldsymbol{v} \cdot \boldsymbol{P}}=\tau \frac{\partial}{\partial t}+y^{a} \frac{\partial}{\partial x^{a}}$, equation (3.16) says we must solve

$$
\tau H+\boldsymbol{y} \cdot \boldsymbol{P}=G\left(\operatorname{ad}_{A}\right) \boldsymbol{v} \cdot \boldsymbol{P}-F\left(\operatorname{ad}_{A}\right)\left(\boldsymbol{\beta} \cdot \boldsymbol{B}+\frac{1}{2} \lambda^{a b} J_{a b}\right),
$$


for $\lambda^{a b}, \boldsymbol{\beta}, \tau$, and $\boldsymbol{y}$ from the components along $J_{a b}, \boldsymbol{B}, H$, and $\boldsymbol{P}$, respectively. The details of the calculation are not particularly illuminating. Let us simply remark that we find

$$
\lambda^{a b}=h_{1}\left(v^{a} x^{b}-v^{b} x^{a}\right)+h_{2}\left(\beta^{a} x^{b}-\beta^{b} x^{a}\right)
$$

for

$$
h_{1}=\frac{G^{-} \frac{\varkappa}{c^{2}}}{1-\frac{1}{x_{+}^{2}}\left(F^{+}-1\right) \frac{\varkappa}{c^{2}} x^{2}} \quad \text { and } \quad h_{2}=\frac{-\frac{1}{x_{+}^{2}}\left(F^{+}-1\right) \frac{\varkappa}{c^{2}} \varsigma t}{1-\frac{1}{x_{+}^{2}}\left(F^{+}-1\right) \frac{\varkappa}{c^{2}} x^{2}} \text {, }
$$

and

$$
\boldsymbol{\beta}=-\frac{G^{-}}{F^{+}} x t \boldsymbol{v}
$$

so that

$$
\lambda^{a b}=-\frac{\varkappa}{c^{2}} \frac{\tanh \left(x_{+} / 2\right)}{x_{+}}\left(v^{a} x^{b}-v^{b} x^{a}\right) .
$$

Re-inserting these expressions into the equation we solve for $\tau$ and $\boldsymbol{y}$, resulting in

$$
\tau=\frac{x_{+} \operatorname{coth} x_{+}-1}{x_{+}^{2}} \frac{\varkappa}{c^{2}} t \boldsymbol{x} \cdot \boldsymbol{v}
$$

and

$$
y^{a}=x_{+} \operatorname{coth}\left(x_{+}\right) v^{a}+\frac{x_{+} \operatorname{coth} x_{+}-1}{x_{+}^{2}} \frac{\varkappa}{c^{2}} \boldsymbol{x} \cdot \boldsymbol{v} x^{a} .
$$

Finally, we have that

$$
\xi_{P_{a}}=\frac{x_{+} \operatorname{coth} x_{+}-1}{x_{+}^{2}} \frac{\varkappa}{c^{2}} x_{a}\left(t \frac{\partial}{\partial t}+x^{b} \frac{\partial}{\partial x^{b}}\right)+x_{+} \operatorname{coth} x_{+} \frac{\partial}{\partial x^{a}} .
$$

Let us summarise all the fundamental vector fields and remember that $x_{+}=$ $\sqrt{-\varkappa\left(\frac{1}{c^{2}} x^{2}+\varsigma t^{2}\right)}$

$$
\begin{aligned}
\xi_{J_{a b}} & =x^{b} \frac{\partial}{\partial x^{a}}-x^{a} \frac{\partial}{\partial x^{b}} \\
\xi_{B_{a}} & =\frac{1}{c^{2}} x^{a} \frac{\partial}{\partial t}-\varsigma t \frac{\partial}{\partial x^{a}} \\
\xi_{H} & =\frac{\partial}{\partial t}+\left(\frac{x_{+} \operatorname{coth} x_{+}-1}{x_{+}^{2}}\right) \varkappa\left(\varsigma t x^{a} \frac{\partial}{\partial x^{a}}-\frac{1}{c^{2}} x^{2} \frac{\partial}{\partial t}\right) \\
\xi_{P_{a}} & =\frac{x_{+} \operatorname{coth} x_{+}-1}{x_{+}^{2}} \frac{\varkappa}{c^{2}} x_{a}\left(t \frac{\partial}{\partial t}+x^{b} \frac{\partial}{\partial x^{b}}\right)+x_{+} \operatorname{coth} x_{+} \frac{\partial}{\partial x^{a}} .
\end{aligned}
$$

We can now calculate the Lie brackets of the vector fields which indeed shows the antihomomorphism with respect to $(5.1)$

$$
\begin{aligned}
& {\left[\xi_{H}, \xi_{\boldsymbol{B}}\right]=-\varsigma \xi_{\boldsymbol{P}}, \quad\left[\xi_{H}, \xi_{\boldsymbol{P}}\right]=\varkappa \xi_{\boldsymbol{B}}, \quad\left[\xi_{\boldsymbol{B}}, \xi_{\boldsymbol{P}}\right]=-\frac{1}{c^{2}} \xi_{H},} \\
& {\left[\xi_{\boldsymbol{B}}, \xi_{\boldsymbol{B}}\right]=\frac{\varsigma}{c^{2}} \xi_{\boldsymbol{J}}, \quad \text { and } \quad\left[\xi_{\boldsymbol{P}}, \xi_{\boldsymbol{P}}\right]=\frac{\varkappa}{c^{2}} \xi_{\boldsymbol{J}} .}
\end{aligned}
$$

Let us emphasise that taking the limit of the vector fields and then calculating their Lie bracket leads to the same result as just taking just the limit of the Lie brackets, i.e., these operations commute. 


\subsection{Soldering form and connection one-form}

The soldering form and the connection one-form are the two components of the pullback of the left-invariant Maurer-Cartan form on $\mathcal{K}$. We will calculate it first for all the (pseudo-)riemannian cases and then take the flat, non-relativistic and ultra-relativistic limit. As we will see, the exponential coordinates are well adapted for that purpose, and the limits can then be systematically studied. That the limits are well defined follows from our construction since the quantities we calculate are a power series of the contraction parameters, $\epsilon=c^{-1}, \varkappa, \tau$ in the $\epsilon \rightarrow 0$ limit and not of their inverse. Let us however stress that for some quantities like, e.g., the galilean structure, modified exponential coordinates are more economical, see appendix A.

For the non-flat (pseudo-)riemannian geometries our exponential coordinates are, except for the hyperbolic case, neither globally valid nor are quantities like the curvature very compact. Since coordinate systems for these cases are well studied, we will focus in the following mainly on the remaining cases. It is useful to derive the soldering form, the invariant connection and the vielbein in full generality since we take the limit and use them to calculate the remaining quantities of interest.

We start by calculating the Maurer-Cartan form via equation (3.45) for which we again use equation (5.25). We find that

$$
\begin{aligned}
\theta+\omega= & d t H+D^{-} \varkappa d t \boldsymbol{x} \cdot \boldsymbol{B}+\frac{1}{x_{+}^{2}}\left(D^{+}-1\right)\left(\varkappa s t d t \boldsymbol{x} \cdot \boldsymbol{P}-\frac{\varkappa}{c^{2}} x^{2} d t H\right) \\
& +D^{+} d \boldsymbol{x} \cdot \boldsymbol{P}+D^{-}\left(-\varkappa t d \boldsymbol{x} \cdot \boldsymbol{B}+\frac{\varkappa}{c^{2}} d x^{a} x^{b} J_{a b}\right) \\
& +\frac{1}{x_{+}^{2}}\left(D^{+}-1\right) \frac{\varkappa}{c^{2}} \boldsymbol{x} \cdot d \boldsymbol{x}(t H+\boldsymbol{x} \cdot \boldsymbol{P}),
\end{aligned}
$$

which, using that

$$
D^{-}=\frac{1-\cosh x_{+}}{x_{+}^{2}}, \quad D^{+}=\frac{\sinh x_{+}}{x_{+}} \quad \text { and hence } \quad \frac{1}{x_{+}^{2}}\left(D^{+}-1\right)=\frac{\sinh x_{+}-x_{+}}{x_{+}^{3}},
$$

gives the following expressions:

$$
\begin{aligned}
\theta= & d t H+\frac{\sinh x_{+}}{x_{+}} d \boldsymbol{x} \cdot \boldsymbol{P} \\
& +\frac{\sinh x_{+}-x_{+}}{x_{+}^{3}} \varkappa\left(\varsigma t d t \boldsymbol{x} \cdot \boldsymbol{P}+\frac{1}{c^{2}}\left(t \boldsymbol{x} \cdot d \boldsymbol{x} H-x^{2} d t H+\boldsymbol{x} \cdot d \boldsymbol{x} \boldsymbol{x} \cdot \boldsymbol{P}\right)\right) \\
\omega= & \frac{1-\cosh x_{+}}{x_{+}^{2}} \varkappa\left(d t \boldsymbol{x} \cdot \boldsymbol{B}-t d \boldsymbol{x} \cdot \boldsymbol{B}-\frac{1}{c^{2}} x^{a} d x^{b} J_{a b}\right) .
\end{aligned}
$$

We can also evaluate the vielbein $E=E_{H} \eta+E_{\boldsymbol{P}} \cdot \boldsymbol{\pi}$ which leads us to

$$
\begin{aligned}
& E_{H}=\frac{\varkappa}{x_{+}^{2}}\left[\left(-\sigma t^{2}-\frac{x^{2}}{c^{2}} x_{+} \operatorname{csch} x_{+}\right) \frac{\partial}{\partial t}+\sigma\left(-1+x_{+} \operatorname{csch} x_{+}\right) t x^{a} \frac{\partial}{\partial x^{a}}\right] \\
& E_{P_{a}}=\frac{\varkappa x^{a}}{c^{2} x_{+}^{2}}\left(-1+x_{+} \operatorname{csch} x_{+}\right)\left(t \frac{\partial}{\partial t}+x^{b} \frac{\partial}{\partial x^{b}}\right)+x_{+} \operatorname{csch} x_{+} \frac{\partial}{\partial x^{a}} .
\end{aligned}
$$




\subsection{Flat limit, Minkowski (M) and euclidean spacetime $(\mathbb{E})$}

In the flat limit $\varkappa \rightarrow 0$ the soldering form and connection one-form are given by

$$
\theta=d t H+d \boldsymbol{x} \cdot \boldsymbol{P} \quad \text { and } \quad \omega=0,
$$

respectively, where $(t, \boldsymbol{x})$ are global coordinates. The vielbein is given by

$$
E=\frac{\partial}{\partial t} \eta+\frac{\partial}{\partial \boldsymbol{x}} \cdot \boldsymbol{\pi}
$$

and the fundamental vector fields, taking the limit of (5.39), by

$$
\xi_{B_{a}}=\frac{1}{c^{2}} x^{a} \frac{\partial}{\partial t}-\varsigma t \frac{\partial}{\partial x^{a}}, \quad \xi_{H}=\frac{\partial}{\partial t}, \quad \text { and } \quad \xi_{P_{a}}=\frac{\partial}{\partial x^{a}} .
$$

Using the soldering form and the vielbein we can now write the metric and co-metric, given in equation (5.7), in coordinates

$$
g=\sigma d t^{2}+\frac{1}{c^{2}} d \boldsymbol{x} \cdot d \boldsymbol{x} \quad \tilde{g}=\frac{1}{c^{2}} \frac{\partial}{\partial t} \otimes \frac{\partial}{\partial t}+\sigma \delta^{i j} \frac{1}{\partial x^{i}} \otimes \frac{1}{\partial x^{j}} .
$$

Since the connection one-form vanishes the torsion and curvature evaluate to

$$
\Omega=0 \quad \Theta=0 .
$$

We can now set $\sigma$ and $c$ to definite values to obtain the Minkowski spacetime $(\sigma=-1$, $c=1)$, Euclidean space $(\sigma=-1, c=1)$, galilean spacetime $\left(\sigma=1, c^{-1}=0\right)$, and carrollian spacetime $(\sigma=0, c=1)$. This is obvious enough for the first two cases so that we go straight to the galilean spacetime.

\subsection{Galilean spacetime (G)}

For galilean spacetimes we have the fundamental vector fields

$$
\xi_{B_{a}}=t \frac{\partial}{\partial x^{a}} \quad \xi_{H}=\frac{\partial}{\partial t} \quad \xi_{P_{a}}=\frac{\partial}{\partial x^{a}},
$$

and the invariant galilean structure which is characterised by the clock one-form $\tau=d t$ and the spatial metric on one-forms $h=\delta^{a b} \frac{\partial}{\partial x^{a}} \otimes \frac{\partial}{\partial x^{b}}$.

\subsection{Carrollian spacetime (C)}

The fundamental vector fields for the carrollian spacetime are

$$
\xi_{B_{a}}=x^{a} \frac{\partial}{\partial t} \quad \xi_{H}=\frac{\partial}{\partial t} \quad \xi_{P_{a}}=\frac{\partial}{\partial x^{a}},
$$

and the invariant carrollian structure is given by $\kappa=\frac{\partial}{\partial t}$ and $b=\delta_{a b} d x^{a} d x^{b}$. 


\subsection{Non-relativistic limit}

In the non-relativistic limit $c \rightarrow \infty$ we get $x_{+}=\sqrt{-\varkappa \varsigma t^{2}}$ and the soldering form and connection one-form are given by

$$
\begin{aligned}
& \theta=d t H+\frac{\sinh x_{+}}{x_{+}} d \boldsymbol{x} \cdot \boldsymbol{P}+\frac{\sinh x_{+}-x_{+}}{x_{+}^{3}} \varkappa s t d t \boldsymbol{x} \cdot \boldsymbol{P} \\
& \omega=\frac{1-\cosh x_{+}}{x_{+}^{2}} \varkappa(d t \boldsymbol{x} \cdot \boldsymbol{B}-t d \boldsymbol{x} \cdot \boldsymbol{B})
\end{aligned} .
$$

We take the non-relativistic limit of the vielbein and obtain

$$
\begin{aligned}
E_{H} & =\frac{\partial}{\partial t}+\left(1-x_{+} \operatorname{csch} x_{+}\right) \frac{x^{a}}{t} \frac{\partial}{\partial x^{a}} \\
E_{P_{a}} & =x_{+} \operatorname{csch} x_{+} \frac{\partial}{\partial x^{a}} .
\end{aligned}
$$

We can now calculate the invariant galilean structure which is given by the clock one-form and the spatial co-metric $\left(h=\varsigma \boldsymbol{P}^{2}\right)$ :

$$
\tau=\eta(\theta)=\sigma d t \quad h=x_{+}^{2} \operatorname{csch}^{2} x_{+} \delta^{a b} \frac{\partial}{\partial x^{a}} \otimes \frac{\partial}{\partial x^{b}} .
$$

The fundamental vector fields are given by

$$
\begin{aligned}
\xi_{B_{a}} & =-\varsigma t \frac{\partial}{\partial x^{a}} \\
\xi_{H} & =\frac{\partial}{\partial t}+\left(\frac{x_{+} \operatorname{coth} x_{+}-1}{x_{+}^{2}}\right) \varkappa \varsigma t x^{a} \frac{\partial}{\partial x^{a}} \\
\xi_{P_{a}} & =x_{+} \operatorname{coth} x_{+} \frac{\partial}{\partial x^{a}} .
\end{aligned}
$$

\subsection{Galilean de Sitter spacetime (dSG)}

We start be setting $\sigma=-1$ and $\varkappa=1$ so that $x_{+}=t$ and see that

$$
\begin{aligned}
& \theta=d t\left(H+\frac{t-\sinh (t)}{t^{2}} \boldsymbol{x} \cdot \boldsymbol{P}\right)+\frac{\sinh (t)}{t} d \boldsymbol{x} \cdot \boldsymbol{P} \\
& \omega=\frac{1-\cosh (t)}{t^{2}}(d t \boldsymbol{x} \cdot \boldsymbol{B}-t d \boldsymbol{x} \cdot \boldsymbol{B}) .
\end{aligned}
$$

The soldering form is invertible for all $(t, \boldsymbol{x})$, since $\sinh (t) / t \neq 0$ for all $t \in \mathbb{R}$. From the above soldering form, it is easily seen that the torsion two-form vanishes and the curvature two-form is given by

$$
\Omega=\frac{1}{t} \sinh (t) B_{a}\left(d t \wedge d x^{a}\right)
$$

The vielbein is given by

$$
E_{H}=\frac{\partial}{\partial t}+(1-t \operatorname{csch} t) \frac{x^{a}}{t} \frac{\partial}{\partial x^{a}} \quad \text { and } \quad E_{P_{a}}=t \operatorname{csch} t \frac{\partial}{\partial x^{a}} .
$$


We can thus find the invariant galilean structure: the clock one-form is given by $\tau=\eta(\theta)=$ $d t$ and the spatial metric is given by

$$
h=t^{2} \operatorname{csch}^{2} t \delta^{a b} \frac{\partial}{\partial x^{a}} \otimes \frac{\partial}{\partial x^{b}} .
$$

Finally, the fundamental vector fields are

$$
\begin{aligned}
\xi_{B_{a}} & =t \frac{\partial}{\partial x^{a}} \\
\xi_{H} & =\frac{\partial}{\partial t}+\left(\frac{1}{t}-\operatorname{coth}(t)\right) x^{a} \frac{\partial}{\partial x^{a}} \\
\xi_{P_{a}} & =t \operatorname{coth}(t) \frac{\partial}{d x^{a}} .
\end{aligned}
$$

\subsection{Galilean anti de Sitter spacetime (AdSG)}

For $\sigma=-1$ and $\varkappa=1$ the soldering form and connection one-form for the canonical invariant connection are

$$
\begin{aligned}
& \theta=d t\left(H+\frac{t-\sin t}{t^{2}} \boldsymbol{x} \cdot \boldsymbol{P}\right)+\frac{\sin t}{t} d \boldsymbol{x} \cdot \boldsymbol{P} \\
& \omega=\frac{1-\cos t}{t^{2}}(t \boldsymbol{x} \cdot \boldsymbol{B}-t d \boldsymbol{x} \cdot \boldsymbol{B}) .
\end{aligned}
$$

Because of the zero of $\sin (t) / t$ at $t= \pm \pi$, the soldering form is an isomorphism for all $\boldsymbol{x}$ and for $t \in(-\pi, \pi)$, so that the exponential coordinates are invalid outside of that region. Let $t_{0} \in(-\pi, \pi)$ and $\boldsymbol{x}_{0} \in \mathbb{R}^{D}$. The orbit of the point $\left(t_{0}, \boldsymbol{x}_{0}\right)$ under the one-parameter subgroup of boosts generated by $\boldsymbol{w} \cdot \boldsymbol{B}$ is

$$
t(s)=t_{0} \quad \text { and } \quad \boldsymbol{x}(s)=\boldsymbol{x}_{0}+s t_{0} \boldsymbol{w} .
$$

The orbits are point-like for $t_{0}=0$ and straight lines for $t_{0} \neq 0$. These orbits remain inside the domain of validity of the exponential coordinates. The generic orbits are, therefore, non-compact.

The torsion two-form again vanishes and the curvature form is

$$
\Omega=\frac{1}{t} \sin t B_{a}\left(d t \wedge d x^{a}\right) .
$$

The vielbein is given by

$$
E_{H}=\frac{\partial}{\partial t}+\left(1-\frac{t}{\sin t}\right) \frac{x^{a}}{t} \frac{\partial}{\partial x^{a}} \quad \text { and } \quad E_{P_{a}}=\frac{t}{\sin t} \frac{\partial}{\partial x^{a}},
$$

so that the invariant galilean structure has a clock one-form $\tau=\eta(\theta)=d t$ and a spatial metric

$$
h=\left(\frac{t}{\sin t}\right)^{2} \delta^{a b} \frac{\partial}{\partial x^{a}} \otimes \frac{\partial}{\partial x^{b}} .
$$

The fundamental vector fields for galilean AdS are

$$
\begin{aligned}
\xi_{B_{a}} & =t \frac{\partial}{\partial x^{a}} \\
\xi_{P_{a}} & =t \cot t \frac{\partial}{\partial x^{a}} \\
\xi_{H} & =\frac{\partial}{\partial t}+\left(\frac{1}{t}-\cot t\right) x^{a} \frac{\partial}{\partial x^{a}} .
\end{aligned}
$$




\subsection{Ultra-relativistic limit}

In the ultra-relativistic limit $\sigma \rightarrow 0$ to the carrollian (anti) de Sitter spacetimes we get $x_{+}=\sqrt{-\frac{\varkappa}{c^{2}} x^{2}}$ and the soldering form and invariant connection are

$$
\begin{aligned}
& \theta=\frac{\sinh x_{+}}{x_{+}}(d t H+d \boldsymbol{x} \cdot \boldsymbol{P})+\left(1-\frac{\sinh x_{+}}{x_{+}}\right) \frac{\boldsymbol{x} \cdot d \boldsymbol{x}}{x^{2}}(t H+\boldsymbol{x} \cdot \boldsymbol{P}) \\
& \omega=\frac{\cosh x_{+}-1}{x^{2}} c^{2}\left(d t \boldsymbol{x} \cdot \boldsymbol{B}-t d \boldsymbol{x} \cdot \boldsymbol{B}-\frac{1}{c^{2}} J_{a b} x^{a} d x^{b}\right) .
\end{aligned}
$$

The vielbein in the ultra-relativistic limit has the following form

$$
\begin{aligned}
E_{H} & =x_{+} \operatorname{csch} x_{+} \frac{\partial}{\partial t} \\
E_{P_{a}} & =\frac{x^{a}}{x^{2}}\left(1-x_{+} \operatorname{csch} x_{+}\right)\left(t \frac{\partial}{\partial t}+x^{b} \frac{\partial}{\partial x^{b}}\right)+x_{+} \operatorname{csch} x_{+} \frac{\partial}{\partial x^{a}} .
\end{aligned}
$$

The ultra-relativistic limit leads to carrollian structure consisting of $\kappa=E_{H}$ and the spatial metric $b=\frac{1}{c^{2}} \boldsymbol{\pi}^{2}$ given by

$$
b=\frac{1}{c^{2}}\left(\frac{\sinh x_{+}}{x_{+}}\right)^{2} d \boldsymbol{x} \cdot d \boldsymbol{x}+\frac{1}{c^{2}}\left(1-\left(\frac{\sinh x_{+}}{x_{+}}\right)^{2}\right) \frac{(\boldsymbol{x} \cdot d \boldsymbol{x})^{2}}{x^{2}}
$$

The fundamental vector fields are

$$
\begin{aligned}
\xi_{B_{a}} & =\frac{1}{c^{2}} x^{a} \frac{\partial}{\partial t} \\
\xi_{H} & =x_{+} \operatorname{coth} x_{+} \frac{\partial}{\partial t} \\
\xi_{P_{a}} & =\frac{x^{a}}{x^{2}}\left(1-x_{+} \operatorname{coth} x_{+}\right)\left(t \frac{\partial}{\partial t}+x^{b} \frac{\partial}{\partial x^{b}}\right)+x_{+} \operatorname{coth} x_{+} \frac{\partial}{\partial x^{a}} .
\end{aligned}
$$

\subsection{2 (Anti) de Sitter carrollian spacetimes (dSC and AdSC)}

We will treat these two spacetimes together, such that $\varkappa=1$ corresponds to carrollian de Sitter (dSC) and $\varkappa=-1$ to carrollian anti de Sitter (AdSC) spacetimes. Furthermore we set $c=1$.

We find that the soldering form is given by

$$
\begin{gathered}
\theta^{(\varkappa=1)}=\frac{\sin |\boldsymbol{x}|}{|\boldsymbol{x}|}(d t H+d \boldsymbol{x} \cdot \boldsymbol{P})+\left(1-\frac{\sin |\boldsymbol{x}|}{|\boldsymbol{x}|}\right) \frac{\boldsymbol{x} \cdot d \boldsymbol{x}}{x^{2}}(t H+\boldsymbol{x} \cdot \boldsymbol{P}) \\
\theta^{(\varkappa=-1)}=\frac{\sinh |\boldsymbol{x}|}{|\boldsymbol{x}|}(d t H+d \boldsymbol{x} \cdot \boldsymbol{P})+\left(1-\frac{\sinh |\boldsymbol{x}|}{|\boldsymbol{x}|}\right) \frac{\boldsymbol{x} \cdot d \boldsymbol{x}}{x^{2}}(t H+\boldsymbol{x} \cdot \boldsymbol{P}) .
\end{gathered}
$$

These soldering forms are invertible whenever the functions $\frac{\sin |x|}{|x|}$ (for $\varkappa=1$ ) or $\frac{\sinh |x|}{|x|}$ (for $\varkappa=-1$ ) are invertible. The latter function is invertible for all $\boldsymbol{x}$, whereas the former function is invertible in the open ball $|\boldsymbol{x}|<\pi$. 
The connection one-form is given by

$$
\begin{gathered}
\omega^{(\varkappa=1)}=\frac{\cos |\boldsymbol{x}|-1}{x^{2}}\left(d t \boldsymbol{x} \cdot \boldsymbol{B}-t d \boldsymbol{x} \cdot \boldsymbol{B}+d x^{a} x^{b} J_{a b}\right) \\
\omega^{(\varkappa=-1)}=\frac{\cosh |\boldsymbol{x}|-1}{x^{2}}\left(d t \boldsymbol{x} \cdot \boldsymbol{B}-t d \boldsymbol{x} \cdot \boldsymbol{B}+d x^{a} x^{b} J_{a b}\right) .
\end{gathered}
$$

The canonical connection is torsion-free, since (A)dSC is symmetric, but it is not flat. The curvature is given by

$$
\begin{aligned}
\Omega^{(\varkappa=1)}= & \left(\frac{\sin |\boldsymbol{x}|}{|\boldsymbol{x}|}\right)^{2} d t \wedge d \boldsymbol{x} \cdot \boldsymbol{B}-\frac{\sin |\boldsymbol{x}|}{|\boldsymbol{x}|}\left(\frac{\sin |\boldsymbol{x}|}{|\boldsymbol{x}|}-1\right) \frac{\boldsymbol{x} \cdot \boldsymbol{B}}{\boldsymbol{x} \cdot \boldsymbol{x}} d t \wedge d \boldsymbol{x} \cdot \boldsymbol{x} \\
& +\left(\frac{\sin |\boldsymbol{x}|}{|\boldsymbol{x}|}\right)^{2} J_{a b} d x^{a} \wedge d x^{b} \\
& +\frac{2 \sin |\boldsymbol{x}|}{|\boldsymbol{x}|}\left(\frac{\sin |\boldsymbol{x}|}{|\boldsymbol{x}|}-1\right)\left(x^{c} x^{b} J_{a c}-t x^{b} B_{a}\right) d x^{a} \wedge d x^{b} \\
\Omega^{(\varkappa=-1)}= & -\left(\frac{\sinh |\boldsymbol{x}|}{|\boldsymbol{x}|}\right)^{2} d t \wedge d \boldsymbol{x} \cdot \boldsymbol{B}+\frac{\sinh |\boldsymbol{x}|}{|\boldsymbol{x}|}\left(\frac{\sinh |\boldsymbol{x}|}{|\boldsymbol{x}|}-1\right) \frac{\boldsymbol{x} \cdot \boldsymbol{B}}{\boldsymbol{x} \cdot \boldsymbol{x}} d t \wedge d \boldsymbol{x} \cdot \boldsymbol{x} \\
& -\left(\frac{\sinh |\boldsymbol{x}|}{|\boldsymbol{x}|}\right)^{2} J_{a b} d x^{a} \wedge d x^{b} \\
& -\frac{2 \sinh |\boldsymbol{x}|}{|\boldsymbol{x}|}\left(\frac{\sinh |\boldsymbol{x}|}{|\boldsymbol{x}|}-1\right)\left(x^{c} x^{b} J_{a c}-t x^{b} B_{a}\right) d x^{a} \wedge d x^{b} .
\end{aligned}
$$

Using the soldering form, we find the vielbein $\mathrm{E}$ to have components

$$
\begin{aligned}
E_{H}^{(\varkappa=1)} & =|\boldsymbol{x}| \csc |\boldsymbol{x}| \frac{\partial}{\partial t} \\
\text { and } \quad E_{P_{a}}^{(\varkappa=1)} & =\frac{x^{a}}{x^{2}}(1-|\boldsymbol{x}| \csc |\boldsymbol{x}|)\left(t \frac{\partial}{\partial t}+x^{b} \frac{\partial}{\partial x^{b}}\right)+|\boldsymbol{x}| \csc |\boldsymbol{x}| \frac{\partial}{\partial x^{a}} \\
E_{H}^{(\varkappa=-1)} & =|\boldsymbol{x}| \operatorname{csch}|\boldsymbol{x}| \frac{\partial}{\partial t} \\
\text { and } \quad E_{P_{a}}^{(\varkappa=-1)} & =\frac{x^{a}}{x^{2}}(1-|\boldsymbol{x}| \operatorname{csch}|\boldsymbol{x}|)\left(t \frac{\partial}{\partial t}+x^{b} \frac{\partial}{\partial x^{b}}\right)+|\boldsymbol{x}| \operatorname{csch}|\boldsymbol{x}| \frac{\partial}{\partial x^{a}}
\end{aligned}
$$

The invariant carrollian structure is given by $\kappa=E_{H}$ and the spatial metric

$$
\begin{aligned}
b^{(\varkappa=1)} & =\left(\frac{\sin |\boldsymbol{x}|}{|\boldsymbol{x}|}\right)^{2} d \boldsymbol{x} \cdot d \boldsymbol{x}+\left(1-\left(\frac{\sin |\boldsymbol{x}|}{|\boldsymbol{x}|}\right)^{2}\right) \frac{(\boldsymbol{x} \cdot d \boldsymbol{x})^{2}}{x^{2}} \\
b^{(\varkappa=-1)} & =\left(\frac{\sinh |\boldsymbol{x}|}{|\boldsymbol{x}|}\right)^{2} d \boldsymbol{x} \cdot d \boldsymbol{x}+\left(1-\left(\frac{\sinh |\boldsymbol{x}|}{|\boldsymbol{x}|}\right)^{2}\right) \frac{(\boldsymbol{x} \cdot d \boldsymbol{x})^{2}}{x^{2}} .
\end{aligned}
$$

Finally, the fundamental vector field of our ultra-relativistic algebras are

$$
\begin{aligned}
\xi_{B_{a}} & =x^{a} \frac{\partial}{\partial t} \\
\xi_{H}^{(\varkappa=1)} & =|\boldsymbol{x}| \cot |\boldsymbol{x}| \frac{\partial}{\partial t}
\end{aligned}
$$




$$
\begin{aligned}
\xi_{H}^{(\varkappa=-1)} & =|\boldsymbol{x}| \operatorname{coth}|\boldsymbol{x}| \frac{\partial}{\partial t} \\
\xi_{P_{a}}^{(\varkappa=1)} & =\frac{x^{a}}{x^{2}}(1-|\boldsymbol{x}| \cot |\boldsymbol{x}|)\left(t \frac{\partial}{\partial t}+x^{b} \frac{\partial}{\partial x^{b}}\right)+|\boldsymbol{x}| \cot |\boldsymbol{x}| \frac{\partial}{\partial x^{a}} \\
\xi_{P_{a}}^{(\varkappa=-1)} & =\frac{x^{a}}{x^{2}}(1-|\boldsymbol{x}| \operatorname{coth}|\boldsymbol{x}|)\left(t \frac{\partial}{\partial t}+x^{b} \frac{\partial}{\partial x^{b}}\right)+|\boldsymbol{x}| \operatorname{coth}|\boldsymbol{x}| \frac{\partial}{\partial x^{a}} .
\end{aligned}
$$

\section{Torsional galilean spacetimes}

Unlike the galilean symmetric spacetimes discussed in section 5, some galilean spacetimes do not arise as limits from the (pseudo-)riemannian spacetimes: namely, the torsional galilean de Sitter $\left(\mathrm{dSG}_{\gamma}\right)$ and anti de Sitter $\left(\mathrm{AdSG}_{\chi}\right)$ spacetimes and spacetime $\mathrm{S} 12_{\gamma, \chi}$, which are the subject of this section. Galilean spacetimes can be seen as null reductions of lorentzian spacetimes one dimension higher and it would be interesting to exhibit these galilean spacetimes as null reductions. We hope to return to this question in the future.

\subsection{Torsional galilean de Sitter spacetime $\left(\mathrm{dSG}_{\gamma \neq 1}\right)$}

The additional brackets not involving $\boldsymbol{J}$ for $\mathrm{dSG}_{\gamma}$ are $[H, \boldsymbol{B}]=-\boldsymbol{P}$ and $[H, \boldsymbol{P}]=\gamma \boldsymbol{B}+$ $(1+\gamma) \boldsymbol{P}$, where $\gamma \in(-1,1)$.

\subsubsection{Fundamental vector fields}

We start by determining the expressions for the fundamental vector fields $\xi_{B_{a}}, \xi_{P_{a}}$, and $\xi_{H}$ relative to the exponential coordinates. The boosts are galilean and hence act in the usual way, with fundamental vector field

$$
\xi_{B_{a}}=t \frac{\partial}{\partial x^{a}}
$$

To determine the other fundamental vector fields we must work harder. The matrix $\operatorname{ad}_{A}$ in this basis is given by

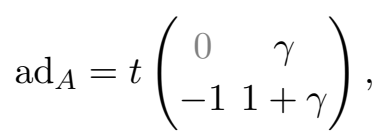

which is diagonalisable (since $\gamma \neq 1$ ) with eigenvalues 1 and $\gamma$, so that $\operatorname{ad}_{A}=S \Delta S^{-1}$, with

$$
\Delta=\left(\begin{array}{cc}
t & 0 \\
0 & t \gamma
\end{array}\right) \quad \text { and } \quad S=\left(\begin{array}{ll}
\gamma & 1 \\
1 & 1
\end{array}\right) \text {. }
$$

Therefore if $f(z)$ is analytic,

$$
f\left(\operatorname{ad}_{A}\right)=S\left(\begin{array}{cc}
f(t) & 0 \\
0 & f(\gamma t)
\end{array}\right) S^{-1}
$$

so that

$$
\begin{aligned}
& f\left(\operatorname{ad}_{A}\right) \boldsymbol{B}=\frac{f(\gamma t)-\gamma f(t)}{1-\gamma} \boldsymbol{B}+\frac{f(\gamma t)-f(t)}{1-\gamma} \boldsymbol{P} \\
& f\left(\operatorname{ad}_{A}\right) \boldsymbol{P}=\frac{\gamma(f(\gamma t)-f(t))}{\gamma-1} \boldsymbol{B}+\frac{\gamma f(\gamma t)-f(t)}{\gamma-1} \boldsymbol{P} .
\end{aligned}
$$


On the other hand, $\operatorname{ad}_{A} H=-\gamma \boldsymbol{x} \cdot \boldsymbol{B}-(1+\gamma) \boldsymbol{x} \cdot \boldsymbol{P}$, so if $f(z)=1+z \widetilde{f}(z)$, then

$$
\begin{aligned}
f\left(\operatorname{ad}_{A}\right) H & =H-\gamma \widetilde{f}\left(\operatorname{ad}_{A}\right) \boldsymbol{x} \cdot \boldsymbol{B}-(1+\gamma) \widetilde{f}\left(\operatorname{ad}_{A}\right) \boldsymbol{x} \cdot \boldsymbol{P} \\
& =H+\frac{\gamma}{1-\gamma}(\gamma \widetilde{f}(\gamma t)-\widetilde{f}(t)) \boldsymbol{x} \cdot \boldsymbol{B}+\frac{1}{1-\gamma}\left(\gamma^{2} \widetilde{f}(\gamma t)-\widetilde{f}(t)\right) \boldsymbol{x} \cdot \boldsymbol{P}
\end{aligned}
$$

where $\widetilde{f}(t)=(f(t)-1) / t$. With these expressions we can now use equation (3.16) to solve for the fundamental vector fields.

Put $X=\boldsymbol{v} \cdot \boldsymbol{P}$ and $Y^{\prime}(0)=\boldsymbol{\beta} \cdot \boldsymbol{B}$ in equation (3.16) to obtain that $\tau=0$ and

$$
\begin{aligned}
\boldsymbol{y} \cdot \boldsymbol{P}= & \frac{1}{\gamma-1}[\gamma(G(\gamma t)-\gamma G(t)) \boldsymbol{v} \cdot \boldsymbol{B}+(\gamma G(\gamma t)-G(t)) \boldsymbol{v} \cdot \boldsymbol{P}] \\
& -\frac{1}{1-\gamma}[(F(\gamma t)-\gamma F(t)) \boldsymbol{\beta} \cdot \boldsymbol{B}+(F(\gamma t)-F(t)) \boldsymbol{\beta} \cdot \boldsymbol{P}] .
\end{aligned}
$$

This requires

$$
\boldsymbol{\beta}=-\gamma \frac{G(\gamma t)-G(t)}{F(\gamma t)-\gamma F(t)} \boldsymbol{v}
$$

and hence, substituting back into the equation for $\boldsymbol{y}$ and simplifying, we obtain

$$
\boldsymbol{y}=t\left(-1+\frac{(\gamma-1) e^{t}}{e^{\gamma t}-e^{t}}\right) \boldsymbol{v}
$$

so that

$$
\xi_{P_{a}}=t\left(-1+\frac{(\gamma-1) e^{t}}{e^{\gamma t}-e^{t}}\right) \frac{\partial}{\partial x^{a}} .
$$

Finally, let $X=H$ and $Y^{\prime}(0)=\boldsymbol{\beta} \cdot \boldsymbol{B}$ in equation (3.16) to obtain that $\tau=1$ and

$$
\begin{aligned}
\boldsymbol{y} \cdot \boldsymbol{P}= & \frac{\gamma}{1-\gamma}(\gamma h(\gamma t)-h(t)) \boldsymbol{x} \cdot \boldsymbol{B}+\frac{1}{1-\gamma}\left(\gamma^{2} h(\gamma t)-h(t)\right) \boldsymbol{x} \cdot \boldsymbol{P} \\
& -\frac{1}{1-\gamma}(F(\gamma t)-\gamma F(t)) \boldsymbol{\beta} \cdot \boldsymbol{B}-\frac{1}{1-\gamma}(F(\gamma t)-F(t)) \boldsymbol{\beta} \cdot \boldsymbol{P},
\end{aligned}
$$

where $h(t)=(G(t)-1) / t$. This requires

$$
\boldsymbol{\beta}=\gamma \frac{\gamma h(\gamma t)-h(t)}{F(\gamma t)-\gamma, F(t)} \boldsymbol{x}
$$

so that

$$
\boldsymbol{y}=\left(1+\frac{1}{t}+\frac{(1-\gamma) e^{t}}{e^{\gamma t}-e^{t}}\right) \boldsymbol{x}
$$

This means that

$$
\xi_{H}=\frac{\partial}{\partial t}+\left(1+\frac{1}{t}+\frac{(1-\gamma) e^{t}}{e^{\gamma t}-e^{t}}\right) x^{a} \frac{\partial}{\partial x^{a}} .
$$

We can easily check that $\left[\xi_{H}, \xi_{B_{a}}\right]=\xi_{P_{a}}$ and $\left[\xi_{H}, \xi_{P_{a}}\right]=-\gamma \xi_{B_{a}}-(1+\gamma) \xi_{P_{a}}$. 


\subsubsection{Soldering form and canonical connection}

This homogeneous spacetime is reductive, so we have not just a soldering form, but also a canonical invariant connection, which can be determined via equation (3.45):

$$
\begin{aligned}
\theta+\omega= & D\left(\operatorname{ad}_{A}\right)(d t H+d \boldsymbol{x} \cdot \boldsymbol{P}) \\
= & d t\left(H+\frac{\gamma}{1-\gamma}(\gamma \widetilde{D}(\gamma t)-\widetilde{D}(t)) \boldsymbol{x} \cdot \boldsymbol{B}+\frac{1}{1-\gamma}\left(\gamma^{2} \widetilde{D}(\gamma t)-\widetilde{D}(t)\right) \boldsymbol{x} \cdot \boldsymbol{P}\right. \\
& +\frac{\gamma}{\gamma-1}(D(\gamma t)-D(t)) d \boldsymbol{x} \cdot \boldsymbol{B}+\frac{1}{\gamma-1}(\gamma D(\gamma t)-D(t)) d \boldsymbol{x} \cdot \boldsymbol{P},
\end{aligned}
$$

where now $\widetilde{D}(z)=(D(z)-1) / z$. Substituting $D(z)=\left(1-e^{-z}\right) / z$, we find that the soldering form is given by

$$
\theta=d t\left(H+\frac{1}{t} \boldsymbol{x} \cdot \boldsymbol{P}\right)+\frac{e^{-t}-e^{-\gamma t}}{t^{2}(1-\gamma)}(d t \boldsymbol{x}-t d \boldsymbol{x}) \cdot \boldsymbol{P},
$$

from where it follows that $\theta$ is invertible for all $(t, \boldsymbol{x})$. The canonical invariant connection is given by

$$
\omega=\left(\frac{1}{t^{2}}+\frac{\gamma e^{-t}-e^{-\gamma t}}{t^{2}(1-\gamma)}\right)(d t \boldsymbol{x}-t d \boldsymbol{x}) \cdot \boldsymbol{B} .
$$

The torsion and curvature of the canonical invariant connection are easily determined from equations (3.47) and (3.48), respectively:

$$
\Theta=\left(\frac{1+\gamma}{1-\gamma}\right) \frac{e^{-t}-e^{-\gamma t}}{t} d t \wedge d \boldsymbol{x} \cdot \boldsymbol{P} \quad \text { and } \quad \Omega=\left(\frac{\gamma}{1-\gamma}\right) \frac{e^{-t}-e^{-\gamma t}}{t} d t \wedge d \boldsymbol{x} \cdot \boldsymbol{B}
$$

This spacetime admits an invariant galilean structure with clock form $\tau=\eta(\theta)=d t$ and spatial metric on one-forms $h=\delta^{a b} E_{P_{a}} \otimes E_{P_{b}}$, where $E$ is the vielbein obtained by inverting the soldering form:

$$
E_{H}=\frac{\partial}{\partial t}+\left(\frac{1}{t}-\frac{\gamma-1}{e^{-t}-e^{-t \gamma}}\right) x^{a} \frac{\partial}{\partial x^{a}} \quad \text { and } \quad E_{P_{a}}=\frac{t(\gamma-1)}{e^{-t}-e^{-\gamma t}} \frac{\partial}{\partial x^{a}}
$$

Therefore, the spatial metric of the galilean structure is given by

$$
h=\frac{t^{2}(\gamma-1)^{2}}{\left(e^{-t}-e^{-\gamma t}\right)^{2}} \delta^{a b} \frac{\partial}{\partial x^{a}} \otimes \frac{\partial}{\partial x^{b}} .
$$

\subsection{Torsional galilean de Sitter spacetime $\left(\mathrm{dSG}_{\gamma=1}\right)$}

This is $\mathrm{dSG}_{1}$, which is the $\gamma \rightarrow 1$ limit of the previous example. Some of the expressions in the previous section have removable singularities at $\gamma=1$, so it seems that treating that case in a separate section leads to a more transparent exposition.

The additional brackets not involving $\boldsymbol{J}$ are now $[H, \boldsymbol{B}]=-\boldsymbol{P}$ and $[H, \boldsymbol{P}]=2 \boldsymbol{P}+\boldsymbol{B}$. We start by determining the expressions for the fundamental vector fields $\xi_{B_{a}}, \xi_{P_{a}}$, and $\xi_{H}$ relative to the exponential coordinates $(t, \boldsymbol{x})$, where $\sigma(t, \boldsymbol{x})=\exp (t H+\boldsymbol{x} \cdot \boldsymbol{P})$. 


\subsubsection{Fundamental vector fields}

The bracket $[H, \boldsymbol{B}]=-\boldsymbol{P}$ shows that $\boldsymbol{B}$ acts as a galilean boost. We can, therefore, immediately write down

$$
\xi_{B_{a}}=t \frac{\partial}{\partial x^{a}} .
$$

To find the other fundamental vector fields requires solving equation (3.16) with $A=$ $t H+\boldsymbol{x} \cdot \boldsymbol{P}$ and $Y^{\prime}(0)=\boldsymbol{\beta} \cdot \boldsymbol{B}$ (for this Lie algebra) for $X=P_{a}$ and $X=H$. To apply equation (3.16) we must first determine how to act with $f\left(\operatorname{ad}_{A}\right)$ on the generators, where $f(z)$ is analytic in $z$.

We start from

$$
\begin{aligned}
& \operatorname{ad}_{A} H=-\boldsymbol{x} \cdot \boldsymbol{B}-2 \boldsymbol{x} \cdot \boldsymbol{P} \\
& \operatorname{ad}_{A} \boldsymbol{P}=2 t \boldsymbol{P}+t \boldsymbol{B} \\
& \operatorname{ad}_{A} \boldsymbol{B}=-t \boldsymbol{P} .
\end{aligned}
$$

It follows from the last two expressions that

$$
\operatorname{ad}_{A}\left(\begin{array}{ll}
\boldsymbol{B} & P
\end{array}\right)=\left(\begin{array}{ll}
\boldsymbol{B} & \boldsymbol{P}
\end{array}\right)\left(\begin{array}{cc}
0 & t \\
-t & 2 t
\end{array}\right),
$$

where the matrix

$$
M=\left(\begin{array}{cc}
0 & 1 \\
-1 & 2
\end{array}\right)
$$

is not diagonalisable, but may be brought to Jordan normal form $M=S J S^{-1}$, where

$$
J=\left(\begin{array}{ll}
1 & 0 \\
1 & 1
\end{array}\right) \quad \text { and } \quad S=S^{-1}=\left(\begin{array}{ll}
1 & -1 \\
0 & -1
\end{array}\right) .
$$

It follows that for $f(z)$ analytic in $z$,

$$
f\left(\operatorname{ad}_{Z}\right)(\boldsymbol{B} \boldsymbol{P})=(\boldsymbol{B} \boldsymbol{P}) S f(t J) S .
$$

If $f(z)=\sum_{n=0}^{\infty} c_{n} z^{n}$,

$$
f(t J)=\sum_{n=0}^{\infty} c_{n} t^{n}\left(\begin{array}{ll}
1 & 0 \\
n & 1
\end{array}\right)=\left(\begin{array}{cc}
f(t) & 0 \\
t f^{\prime}(t) & f(t)
\end{array}\right) .
$$

Performing the matrix multiplication, we arrive at

$$
\begin{aligned}
& \left.f\left(\operatorname{ad}_{A}\right) \boldsymbol{B}=\left(f(t)-t f^{\prime}(t)\right) \boldsymbol{B}-t f^{\prime}(t)\right) \boldsymbol{P} \\
& f\left(\operatorname{ad}_{A}\right) \boldsymbol{P}=t f^{\prime}(t) \boldsymbol{B}+\left(f(t)+t f^{\prime}(t)\right) \boldsymbol{P} .
\end{aligned}
$$

Similarly,

$$
f\left(\operatorname{ad}_{A}\right) H=f(0) H-2 \boldsymbol{x} \cdot \widetilde{f}\left(\operatorname{ad}_{A}\right) \boldsymbol{P}-\boldsymbol{x} \cdot \widetilde{f}\left(\operatorname{ad}_{A}\right) \boldsymbol{B},
$$

where $\tilde{f}(z)=(f(z)-f(0)) / z$. 
We are now ready to apply equation (3.16). Let $X=\boldsymbol{v} \cdot \boldsymbol{P}$. Then equation (3.16) becomes

$$
\begin{aligned}
\tau H+\boldsymbol{y} \cdot \boldsymbol{P} & =G\left(\operatorname{ad}_{A}\right) \boldsymbol{v} \cdot \boldsymbol{P}-F\left(\operatorname{ad}_{A}\right) \boldsymbol{\beta} \cdot \boldsymbol{B} \\
& =\left(G(t)+t G^{\prime}(t)\right) \boldsymbol{v} \cdot \boldsymbol{P}+t G^{\prime}(t) \boldsymbol{v} \cdot \boldsymbol{B}-\left(F(t)-t F^{\prime}(t)\right) \boldsymbol{\beta} \cdot \boldsymbol{B}+t F^{\prime}(t) \boldsymbol{\beta} \cdot \boldsymbol{P},
\end{aligned}
$$

from where we find that $\tau=0$,

$$
\begin{aligned}
\boldsymbol{\beta} & =\frac{t G^{\prime}(t)}{F(t)-t F^{\prime}(t)} \boldsymbol{v} \\
\text { and hence } \quad \boldsymbol{y} & =\frac{F(t) G(t)+t\left(F(t) G^{\prime}(t)-F^{\prime}(t) G(t)\right)}{F(t)-t F^{\prime}(t)} \boldsymbol{v}=(1-t) \boldsymbol{v},
\end{aligned}
$$

so that

$$
\xi_{P_{a}}=(1-t) \frac{\partial}{\partial x^{a}}
$$

which is indeed the limit $\gamma \rightarrow 1$ of equation (6.10).

Now let $X=H$, so that equation (3.16) becomes

$$
\begin{aligned}
\tau H+\boldsymbol{y} \cdot \boldsymbol{P}= & G\left(\operatorname{ad}_{A}\right) H-\boldsymbol{\beta} \cdot F\left(\operatorname{ad}_{A}\right) \boldsymbol{B} \\
= & H-2 \boldsymbol{x} \cdot \widetilde{G}\left(\operatorname{ad}_{A}\right) \boldsymbol{P}-\boldsymbol{x} \cdot \widetilde{G}\left(\operatorname{ad}_{A}\right) \boldsymbol{B}-\boldsymbol{\beta} \cdot F\left(\operatorname{ad}_{A}\right) \boldsymbol{B} \\
= & H-\left(\widetilde{G}(t)+t \widetilde{G}^{\prime}(t)\right) \boldsymbol{x} \cdot \boldsymbol{B}-\left(F(t)-t F^{\prime}(t)\right) \boldsymbol{\beta} \cdot \boldsymbol{B} \\
& -\left(2 \widetilde{G}(t)+t \widetilde{G}^{\prime}(t)\right) \boldsymbol{x} \cdot \boldsymbol{P}+t F^{\prime}(t) \boldsymbol{\beta} \cdot \boldsymbol{P},
\end{aligned}
$$

from where $\tau=1$,

$$
\boldsymbol{\beta}=\frac{\widetilde{G}(t)+t \widetilde{G}^{\prime}(t)}{t F^{\prime}(t)-F(t)} \boldsymbol{x} \quad \text { and hence } \quad \boldsymbol{y}=\frac{t\left(F^{\prime}(t) \widetilde{G}(t)-F(t) \widetilde{G}^{\prime}(t)\right)-2 F(t) \widetilde{G}(t)}{F(t)-t F^{\prime}(t)} \boldsymbol{x}=\boldsymbol{x} .
$$

In summary,

$$
\xi_{H}=\frac{\partial}{\partial t}+x^{a} \frac{\partial}{\partial x^{a}},
$$

which is indeed the $\gamma \rightarrow 1$ limit of equation (6.14).

\subsubsection{Soldering form and canonical connection}

To calculate the soldering form and the connection one-form for the canonical invariant connection, we apply equation (3.45):

$$
\begin{aligned}
\sigma^{*} \vartheta= & D\left(\operatorname{ad}_{A}\right)(d t H+d \boldsymbol{x} \cdot \boldsymbol{P}) \\
= & d t\left(H-2 \boldsymbol{x} \cdot \widetilde{D}\left(\operatorname{ad}_{A}\right) \boldsymbol{P}-\boldsymbol{x} \cdot \widetilde{D}\left(\operatorname{ad}_{A}\right) \boldsymbol{B}\right)+d \boldsymbol{x} \cdot D\left(\operatorname{ad}_{A}\right) \boldsymbol{P} \\
= & d t\left(H-\left(\widetilde{D}(t)+t \widetilde{D}^{\prime}(t)\right) \boldsymbol{x} \cdot \boldsymbol{B}-\left(2 \widetilde{D}(t)+t \widetilde{D}^{\prime}(t)\right) \boldsymbol{x} \cdot \boldsymbol{P}\right) \\
& +\left(D(t)+t D^{\prime}(t)\right) d \boldsymbol{x} \cdot \boldsymbol{P}+t D^{\prime}(t) d \boldsymbol{x} \cdot \boldsymbol{B} .
\end{aligned}
$$


Performing the calculation,

$$
\begin{aligned}
\theta & =d t\left(H+\frac{1-e^{-t}}{t} \boldsymbol{x} \cdot \boldsymbol{P}\right)+e^{-t} d \boldsymbol{x} \cdot \boldsymbol{P} \\
\omega & =\frac{1}{t}\left(\frac{1-e^{-t}}{t}-e^{-t}\right)(\boldsymbol{x} \cdot \boldsymbol{B} d t-t d \boldsymbol{x} \cdot \boldsymbol{B}),
\end{aligned}
$$

which are equations (6.16) and (6.17) in the limit $\gamma \rightarrow 1$. Notice that $\theta$ is an isomorphism for all $(t, \boldsymbol{x})$.

The torsion and curvature two-forms for the canonical invariant connection are given by

$$
\Theta=-2 e^{-t} d t \wedge d \boldsymbol{x} \cdot \boldsymbol{P} \quad \text { and } \quad \Omega=-e^{-t} d t \wedge d \boldsymbol{x} \cdot \boldsymbol{B} .
$$

The vielbein $E$ has components

$$
E_{H}=\frac{\partial}{\partial t}+\frac{1-e^{t}}{t} x^{a} \frac{\partial}{\partial x^{a}} \quad \text { and } \quad E_{P_{a}}=e^{t} \frac{\partial}{\partial x^{a}} .
$$

The invariant galilean structure has clock form $\tau=\eta(\theta)=d t$ and inverse spatial metric

$$
h=\delta^{a b} E_{P_{a}} \otimes E_{P_{b}}=e^{2 t} \delta^{a b} \frac{\partial}{\partial x^{a}} \otimes \frac{\partial}{\partial x^{b}} .
$$

\subsection{Torsional galilean anti de Sitter spacetime $\left(\mathrm{AdSG}_{\chi}\right)$}

Here $[H, \boldsymbol{B}]=-\boldsymbol{P}$ and $[H, \boldsymbol{P}]=\left(1+\chi^{2}\right) \boldsymbol{B}+2 \chi \boldsymbol{P}$.

\subsubsection{Fundamental vector fields}

Since $\boldsymbol{B}$ acts via galilean boosts we can immediately write down

$$
\xi_{B_{a}}=t \frac{\partial}{\partial x^{a}}
$$

To calculate the other fundamental vector fields we employ equation (3.16). The adjoint action of $A=t H+\boldsymbol{x} \cdot \boldsymbol{P}$ is given by

$$
\begin{aligned}
& \operatorname{ad}_{A} H=-\left(1+\chi^{2}\right) \boldsymbol{x} \cdot \boldsymbol{B}-2 \chi \boldsymbol{x} \cdot \boldsymbol{P} \\
& \operatorname{ad}_{A} \boldsymbol{B}=-t \boldsymbol{P} \\
& \operatorname{ad}_{A} \boldsymbol{P}=t\left(1+\chi^{2}\right) \boldsymbol{B}+2 t \chi \boldsymbol{P} .
\end{aligned}
$$

In matrix form,

$$
\operatorname{ad}_{A}\left(\begin{array}{ll}
\boldsymbol{B} & \boldsymbol{P}
\end{array}\right)=\left(\begin{array}{ll}
\boldsymbol{B} & \boldsymbol{P}
\end{array}\right)\left(\begin{array}{cc}
0 & \left(1+\chi^{2}\right) t \\
-t & 2 t \chi
\end{array}\right)
$$

We notice that this matrix is diagonalisable:

$$
\left(\begin{array}{cc}
0 & \left(1+\chi^{2}\right) \\
-1 & 2 \chi
\end{array}\right)=S \Delta S^{-1}, \quad \text { where } \quad S:=\left(\begin{array}{cc}
\chi+i \chi-i \\
1 & 1
\end{array}\right) \quad \text { and } \quad \Delta:=\left(\begin{array}{cc}
\chi-i & 0 \\
0 & \chi+i
\end{array}\right) \text {. }
$$


So if $f(z)$ is analytic in $z$,

$$
f\left(\operatorname{ad}_{A}\right)(\boldsymbol{B} \boldsymbol{P})=(\boldsymbol{B} \boldsymbol{P}) S f(t \Delta) S^{-1},
$$

or letting $t_{ \pm}:=t(\chi \pm i)$,

$$
\begin{aligned}
f\left(\operatorname{ad}_{A}\right) \boldsymbol{B} & =\frac{i}{2}\left(f\left(t_{+}\right)-f\left(t_{-}\right)\right)(\boldsymbol{P}+\chi \boldsymbol{B})+\frac{1}{2}\left(f\left(t_{+}\right)+f\left(t_{-}\right)\right) \boldsymbol{B} \\
f\left(\operatorname{ad}_{A}\right) \boldsymbol{P} & =-\frac{i}{2}\left(f\left(t_{+}\right)-f\left(t_{-}\right)\right)\left(\chi \boldsymbol{P}+\left(1+\chi^{2}\right) \boldsymbol{B}\right)+\frac{1}{2}\left(f\left(t_{+}\right)+f\left(t_{-}\right)\right) \boldsymbol{P} .
\end{aligned}
$$

Similarly,

$$
\begin{aligned}
f\left(\operatorname{ad}_{A}\right) H & =f(0) H+\frac{1}{\operatorname{ad}_{A}}\left(f\left(\operatorname{ad}_{A}\right)-f(0)\right) \operatorname{ad}_{A} H \\
& =f(0) H-\left(1+\chi^{2}\right) \boldsymbol{x} \cdot \widetilde{f}\left(\operatorname{ad}_{A}\right) \boldsymbol{B}-2 \chi \boldsymbol{x} \cdot \widetilde{f}\left(\operatorname{ad}_{A}\right) \boldsymbol{P},
\end{aligned}
$$

where $\widetilde{f}(z):=(f(z)-f(0)) / z$. With these formulae we can now use equation (3.16) to find out the expressions for the fundamental vector fields $\xi_{H}$ and $\xi_{P_{a}}$. Putting $X=\boldsymbol{v} \cdot \boldsymbol{P}$ and $Y^{\prime}(0)=\boldsymbol{\beta} \cdot \boldsymbol{B}$ in equation (3.16) we arrive at

$$
\boldsymbol{\beta}=\frac{-i\left(1+\chi^{2}\right)\left(G\left(t_{+}\right)-G\left(t_{-}\right)\right)}{F\left(t_{+}\right)+F\left(t_{-}\right)+i \chi\left(F\left(t_{+}\right)-F\left(t_{-}\right)\right)} \boldsymbol{v}
$$

and hence

$$
\xi_{P_{a}}=t(\cot t-\chi) \frac{\partial}{\partial x^{a}} .
$$

Similarly, putting $X=H$ and $Y^{\prime}(0)=\boldsymbol{\beta} \cdot \boldsymbol{B}$ in equation (3.16) we find

$$
\boldsymbol{\beta}=\frac{i \chi\left(\widetilde{G}\left(t_{+}\right)-\widetilde{G}\left(t_{-}\right)\right)-\left(\widetilde{G}\left(t_{+}\right)+\widetilde{G}\left(t_{-}\right)\right)}{F\left(t_{+}\right)+F\left(t_{-}\right)+i \chi\left(F\left(t_{+}\right)-F\left(t_{-}\right)\right)} \boldsymbol{x}
$$

and hence

$$
\xi_{H}=\frac{\partial}{\partial t}+\left(\frac{1}{t}+\chi-\cot t\right) x^{a} \frac{\partial}{\partial x^{a}} .
$$

We check that $\left[\xi_{H}, \xi_{B_{a}}\right]=\xi_{P_{a}}$ and $\left[\xi_{H}, \xi_{P_{a}}\right]=-\left(1+\chi^{2}\right) \xi_{B_{a}}-2 \chi \xi_{P_{a}}$, as expected. Another check is that taking $\chi \rightarrow 0$, we recover the fundamental vector fields for galilean anti de Sitter spacetime given by equation (5.69).

\subsubsection{Soldering form and canonical connection}

Let us now use equation (3.45) to calculate the soldering form $\theta$ and the connection oneform $\omega$ for the canonical invariant connection:

$$
\begin{aligned}
\theta+\omega & =D\left(\operatorname{ad}_{A}\right)(d t H+d \boldsymbol{x} \cdot \boldsymbol{P}) \\
& =d t\left(H-\left(1+\chi^{2}\right) \boldsymbol{x} \cdot \widetilde{D}\left(\operatorname{ad}_{A}\right) \boldsymbol{B}-2 \chi \boldsymbol{x} \cdot \widetilde{D}\left(\operatorname{ad}_{A}\right) \boldsymbol{P}\right)+d \boldsymbol{x} \cdot D\left(\operatorname{ad}_{A}\right) \boldsymbol{P},
\end{aligned}
$$

where $\widetilde{D}(z)=(D(z)-1) / z$. Evaluating these expressions, we find

$$
\theta=d t\left(H+\frac{\left(t-e^{\chi t} \sin t\right)}{t^{2}} \boldsymbol{x} \cdot \boldsymbol{P}\right)+\frac{1}{t} e^{-\chi t} \sin t d \boldsymbol{x} \cdot \boldsymbol{P}
$$


and

$$
\omega=\frac{1-e^{-\chi t}(\cos t+\chi \sin t)}{t^{2}}(d t \boldsymbol{x} \cdot \boldsymbol{B}-t d \boldsymbol{x} \cdot \boldsymbol{B}) .
$$

Again, the zeros of $\frac{e^{-\chi t} \sin t}{t}$ at $t= \pm \pi$ invalidate the exponential coordinates for $t \notin(-\pi, \pi)$.

The torsion and curvature of the canonical invariant connection are easily calculated to be

$$
\begin{aligned}
& \Theta=-\frac{2 \chi}{t} e^{-\chi t} \sin t d t \wedge d \boldsymbol{x} \cdot \boldsymbol{P} \\
& \Omega=-\frac{\left(1+\chi^{2}\right)}{t} e^{-\chi t} \sin t d t \wedge d \boldsymbol{x} \cdot \boldsymbol{B} .
\end{aligned}
$$

As $\chi \rightarrow 0$, the torsion vanishes and the curvature agrees with that of the galilean anti de Sitter spacetime (S10) in equation (5.66).

The vielbein $E$ has components

$$
\begin{aligned}
& E_{H}=\frac{\partial}{\partial t}+\left(\frac{1}{t}-e^{\chi t} \csc t\right) x^{a} \frac{\partial}{\partial x^{a}} \\
& E_{P_{a}}=t e^{\chi t} \csc t \frac{\partial}{\partial x^{a}}
\end{aligned}
$$

whose $\chi \rightarrow 0$ limit agrees with equation (5.67). The invariant galilean structure has clock form $\tau=\eta(\theta)=d t$ and inverse spatial metric

$$
h=t^{2} e^{2 \chi t} \csc ^{2} t \delta^{a b} \frac{\partial}{\partial x^{a}} \otimes \frac{\partial}{\partial x^{b}},
$$

which again agrees with equation (5.68) in the limit $\chi \rightarrow 0$.

\subsection{Spacetime $\mathrm{S12}_{\gamma, \chi}$}

There is a two-parameter family of spacetimes which is unique to $D=2$. Here the additional brackets are $[H, \boldsymbol{B}]=-\boldsymbol{P}$, and $[H, \boldsymbol{P}]=(1+\gamma) \boldsymbol{P}-\chi \widetilde{\boldsymbol{P}}+\gamma \boldsymbol{B}-\chi \tilde{\boldsymbol{B}}$. To make the following calculations easier we may complexify the algebra by defining $\mathbb{P}=\boldsymbol{P}_{1}+i \boldsymbol{P}_{2}$ and $\mathbb{B}=\boldsymbol{B}_{1}+i \boldsymbol{B}_{2}$ such that the brackets become $[H, \mathbb{B}]=-\mathbb{P},[H, \mathbb{P}]=(1+z) \mathbb{P}+z \mathbb{B}$, where $z=\gamma+i \chi$. We start by determining the expressions for the fundamental vector fields $\xi_{B_{a}}$, $\xi_{P_{a}}$, and $\xi_{H}$.

\subsubsection{Fundamental vector fields}

Since $\boldsymbol{B}$ acts via galilean boosts we can immediately write down

$$
\xi_{B_{a}}=t \frac{\partial}{\partial x^{a}}
$$

To calculate the other fundamental vector fields we employ equation (3.16). The adjoint action of $A=t H+\boldsymbol{x} \cdot \boldsymbol{P}$ on a basis $(\mathbb{B}, \mathbb{P})$ is given by

$$
\operatorname{ad}_{A}=t\left(\begin{array}{cc}
0 & z \\
-1 & 1+z
\end{array}\right)
$$


Notice that this matrix is diagonalisable:

$$
\left(\begin{array}{cc}
0 & z \\
-1 & 1+z
\end{array}\right)=S \Delta S^{-1}, \quad \text { where } \quad S:=\left(\begin{array}{cc}
z & 1 \\
1 & 1
\end{array}\right) \quad \text { and } \quad \Delta:=\left(\begin{array}{ll}
1 & 0 \\
0 & z
\end{array}\right) .
$$

So if $f(\zeta)$ is an analytic function of $\zeta$,

$$
f\left(\operatorname{ad}_{A}\right)(\mathbb{B} \mathbb{P})=(\mathbb{B} \mathbb{P}) S f(t \Delta) S^{-1},
$$

such that

$$
\begin{aligned}
& f\left(\operatorname{ad}_{A}\right) \mathbb{B}=\frac{f(z t)-z f(t)}{1-z} \mathbb{B}+\frac{f(z t)-f(t)}{1-z} \mathbb{P} \\
& f\left(\operatorname{ad}_{A}\right) \mathbb{P}=\frac{z(f(t)-f(z t))}{1-z} \mathbb{B}+\frac{f(t)-z f(z t)}{1-z} \mathbb{P} .
\end{aligned}
$$

Let $\widetilde{f}(\zeta):=(f(\zeta)-f(0)) / \zeta$. Then we may write, using the notation $x=x^{1}+i x^{2}$,

$$
\begin{aligned}
\operatorname{ad}_{A} H & =-\frac{1}{t} \operatorname{Re}\left(\bar{\nwarrow} \operatorname{ad}_{A} \mathbb{P}\right) \\
f\left(\operatorname{ad}_{A}\right) H & =H-\operatorname{Re}\left(\bar{\ltimes} \widetilde{f}\left(\operatorname{ad}_{A}\right)(z \mathbb{B}+(1+z) \mathbb{P})\right) .
\end{aligned}
$$

Similarly, let $\vee, \beta$, and $y$ now be complex numbers. Setting $X=\operatorname{Re}(\overline{\mathbb{v}} \mathbb{P})$ and $Y^{\prime}(0)=$ $\operatorname{Re}(\bar{\beta} \mathbb{B})$ we obtain $\tau=0$ and

$$
\begin{array}{r}
\operatorname{Re}(\bar{y} \mathbb{P})=\operatorname{Re}\left(\bar{v} \frac{1}{1-z}(z(G(t)-G(z t)) \mathbb{B}+(G(t)-z G(z t)) \mathbb{P})\right. \\
\left.-\bar{\beta} \frac{1}{z-1}((z F(t)-F(z t)) \mathbb{B}+(F(t)-F(z t)) \mathbb{P})\right) .
\end{array}
$$

This requires

$$
\bar{\beta}=\frac{z(G(z t)-G(t))}{z F(t)-F(z t)} \bar{\nabla} .
$$

Substituting back into the equation we find

$$
y=t\left(-1+\frac{e^{t}(-1+\gamma-i \chi)}{-e^{t}+e^{t(\gamma-i \chi)}}\right) \vee=:(a+i b) \vee,
$$

where we have introduced $a$ and $b$ as the real and imaginary parts of the expression multiplying $\vee$. In full glory,

$$
\begin{aligned}
a & =\frac{t((\gamma-1) \cos (t \gamma)+(1+\gamma) \cosh (t(\gamma-1))-\chi \sin (t \chi)+(\gamma-1) \sinh (t(1-\gamma)))}{2(\cos (t \chi)-\cosh (t(\gamma-1)))} \\
b & =\frac{t\left(\chi \cos (t \chi)+(1-\gamma) \sin (t \chi)-e^{t(1-\gamma)} \chi\right)}{2(\cos (t \chi)-\cosh (t(\gamma-1)))}
\end{aligned}
$$

so that $y^{a}=a v^{a}-b \epsilon_{a b} v^{b}$ and hence

$$
\xi_{P_{a}}=a \frac{\partial}{\partial x^{a}}+b \epsilon_{a b} \frac{\partial}{\partial x^{b}} .
$$


Now letting $X=H$ and $Y^{\prime}(0)=\operatorname{Re}(\bar{\beta} \mathbb{B})$, we obtain $\tau=1$ and

$$
\begin{aligned}
\operatorname{Re}(\overline{\mathrm{y}} \mathbb{P})= & -\operatorname{Re}\left(\overline{\bar{x}} \widetilde{G}\left(\operatorname{ad}_{A}\right)((1+z) \mathbb{P}+z \mathbb{B})-\operatorname{Re}\left(\bar{\beta} F\left(\operatorname{ad}_{A}\right) \mathbb{B}\right)\right. \\
= & -\operatorname{Re}\left(\frac{\overline{\widetilde{K}}(1+z)}{1-z}(z(\widetilde{G}(t)-\widetilde{G}(z t)) \mathbb{B}+(\widetilde{G}(t)-z \widetilde{G}(z t)) \mathbb{P})\right) \\
& -\operatorname{Re}\left(\frac{\overline{\boldsymbol{K}} z}{1-z}((\widetilde{G}(z t)-z \widetilde{G}(t)) \mathbb{B}+(\widetilde{G}(z t)-\widetilde{G}(t)) \mathbb{P})\right) \\
& -\operatorname{Re}\left(\frac{\bar{\beta}}{1-z}((F(z t)-z F(t)) \mathbb{B}+(F(z t)-F(t)) \mathbb{P})\right) .
\end{aligned}
$$

We solve for $\beta$ to find

$$
\bar{\beta}=\frac{z(z \widetilde{G}(z t)-\widetilde{G}(t))}{F(z t)-z F(t)} \overline{\mathrm{x}} .
$$

Substituting this back in to the equation, we find

$$
y=\left(1+\frac{1}{t}+\frac{(-1+\gamma-i \chi) e^{t}}{\left.e^{t}-e^{(\gamma-i \chi) t}\right)}\right) \ltimes=:(c+i d) \ltimes,
$$

where $c, d$ are the real and imaginary parts of the expression multiplying $x$. Expanding we find

$$
\begin{aligned}
& c=\frac{e^{2 \gamma t}(1+t)+e^{2 t}(1+t \gamma)-e^{t(1+\gamma)}(2+t(1+\gamma) \cos (t \chi)+t \chi \sin (t \chi))}{t\left(e^{2 t}+e^{2 t \gamma}-2 e^{t(1+\gamma)} \cos (t \chi)\right)} \\
& d=\frac{-e^{2 t} \chi+e^{t(1+\gamma)}(\chi \cos (t \chi)+(1-\gamma) \sin (t \chi))}{t\left(e^{2 t}+e^{2 t \gamma}-2 e^{t(1+\gamma)} \cos (t \chi)\right)}
\end{aligned}
$$

so that $y^{a}=c x^{a}-d \epsilon_{a b} x^{b}$ and hence

$$
\xi_{H}=\frac{\partial}{\partial t}+c x^{a} \frac{\partial}{\partial x^{a}}+d \epsilon_{a b} x^{a} \frac{\partial}{\partial x^{b}} .
$$

One can check that $\left[\xi_{H}, \xi_{P_{a}}\right]=\xi_{\left[P_{a}, H\right]}$ and $\left[\xi_{H}, \xi_{B_{a}}\right]=\xi_{\left[B_{a}, H\right]}$.

\subsubsection{Soldering form and canonical connection}

We can now use equation (3.45) in order to calculate the soldering form $\theta$ and the connection one-form $\omega$ for the canonical invariant connection:

$$
\begin{aligned}
\theta+\omega & =D\left(\operatorname{ad}_{A}\right)(d t H+d \boldsymbol{x} \cdot \boldsymbol{P}) \\
& =d t H-d t \operatorname{Re}\left(\overline{\bar{\alpha}} \widetilde{D}\left(\operatorname{ad}_{A}\right)(z \mathbb{B}+(1+z) \mathbb{P})\right)+\operatorname{Re}\left(d \overline{\bar{\alpha}} D\left(\operatorname{ad}_{A}\right) \mathbb{P}\right),
\end{aligned}
$$

where $\widetilde{D}(\zeta)=(D(\zeta)-1) / \zeta$. Evaluating these expressions we find

$$
\theta=d t H+\frac{d t}{t} \operatorname{Re}(\overline{\bar{x}} \mathbb{P})+\operatorname{Re}\left(\frac{t d \overline{\bar{x}}-\bar{x} d t}{t^{2}(z-1)}\left(e^{-t}-e^{-t z}\right) \mathbb{P}\right)
$$

and

$$
\omega=\operatorname{Re}\left(\frac{\bar{x} d t-t d \bar{\not}}{t^{2}}\left(1+\frac{e^{-t z}-z e^{-t}}{z-1}\right) \mathbb{B}\right) .
$$


It is not immediately obvious from the expression for $\theta$ whether it fails to be an isomorphism. Because $\theta_{t}^{H}=1$, the soldering form is invertible provided that the determinant of $\theta_{b}^{P_{a}}$ does not vanish. Unpacking the complex notation, we find that the determinant is given by

$$
\frac{e^{-2 t(\gamma+1)}\left(e^{2 t \gamma}+e^{2 t}-2 e^{t(\gamma+1)} \cos (t \chi)\right)}{t^{2}\left((\gamma-1)^{2}+\chi^{2}\right)} .
$$

This is nowhere zero for $\gamma \in[-1,1)$. But if $\gamma=1$, then it becomes

$$
\frac{2 e^{-2 t}(1-\cos (t \chi))}{t^{2} \chi^{2}}
$$

which vanishes whenever $t \chi=2 \pi k, k= \pm 1, \pm 2, \cdots$. Therefore, for $\chi>0$ and $\gamma \in[-1,1)$, the soldering form is invertible everywhere, whereas if $\gamma=1$ then it is invertible for $t \in\left(-\frac{2 \pi}{\chi}, \frac{2 \pi}{\chi}\right)$ and for all $\boldsymbol{x} \in \mathbb{R}^{2}$. For $\chi=0$, the soldering form is invertible everywhere. This agrees with $\mathrm{dSG}_{\gamma}$ and $\mathrm{AdSG}_{2 / \chi}$, which are the $\chi \rightarrow 0$ and $\gamma \rightarrow 1$ limits of $\mathrm{S}_{2} 2_{\gamma, \chi}$, respectively. ${ }^{10}$

The torsion and curvature of the canonical invariant connection are calculated to be

$$
\begin{aligned}
& \Theta=-\operatorname{Re}\left(\frac{1+z}{t(z-1)}\left(e^{-t}-e^{-t z}\right) d t \wedge d \overline{\bar{\alpha}} \mathbb{P}\right) \\
& \Omega=-\operatorname{Re}\left(\frac{z}{t(z-1)}\left(e^{-t}-e^{-t z}\right) d t \wedge d \overline{\bar{\alpha}} \mathbb{B}\right) .
\end{aligned}
$$

Using the soldering form we can read-off the vielbein and deduce the invariant galilean structure. The clock one-form is $\tau=\eta(\theta)=d t$ and the inverse spatial metric

$$
h=\left(\operatorname{Re}\left(\frac{(z-1) t}{e^{-t}-e^{-z t}}\right)\right)^{2} \delta^{a b} \frac{\partial}{\partial x^{a}} \otimes \frac{\partial}{\partial x^{b}} .
$$

\subsection{The action of the boosts}

In this section we show that the generic orbits of boosts are not compact in the torsional galilean spacetimes discussed above. This requires a different argument to the ones we used for the symmetric spaces.

Let $M$ be one of the torsional galilean spacetimes discussed in this section; that is,

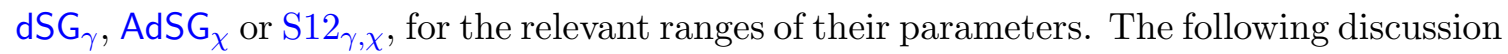
applies verbatim to the torsional galilean (anti) de Sitter, whereas for S12 $2_{\gamma, \chi}$ the exposition is more cumbersome; although, as we will see, the result still holds.

Our default description of $M$ is as a simply-connected kinematical homogeneous spacetime $\mathcal{K} / \mathcal{H}$, where $\mathcal{K}$ is a simply-connected kinematical Lie group and $\mathcal{H}$ is the connected subgroup generated by the boots and rotations. Our first observation is that we may dispense with the rotations and also describe $M$ as $\mathcal{S} / \mathcal{B}$, where $\mathcal{S}$ is the simply-connected solvable Lie group generated by the boosts and spatio-temporal translations and $\mathcal{B}$ is the

\footnotetext{
${ }^{10}$ One might ask why in $\mathrm{AdSG}_{2 / \chi}$ the range of $t$ does not involve $\chi$ but here it does. It has to do with the complex change of basis which gives the isomorphism $\mathrm{S} 12_{1, \chi} \cong \mathrm{AdSG}_{2 / \chi}$.
} 
connected abelian subgroup generated by the boosts. The Lie algebra $\mathfrak{s}$ of $\mathcal{S}$ is spanned by $H, B_{a}, P_{a}$ and the Lie algebra $\mathfrak{b}$ of $\mathcal{B}$ is spanned by $B_{a}$ with non-zero brackets

$$
\left[H, B_{a}\right]=-P_{a} \quad \text { and } \quad\left[H, P_{a}\right]=\alpha B_{a}+\beta P_{a},
$$

for some real numbers $\alpha, \beta$ depending on the parameters $\gamma, \chi$. We may identify $\mathfrak{s}$ with the Lie subalgebra of $\mathfrak{g l}(2 D+1, \mathbb{R})$ given by

$$
\mathfrak{s}=\left\{\left(\begin{array}{ccc}
0 & t \alpha \mathbb{1} & \boldsymbol{y} \\
-t \mathbb{1} & t \beta \mathbb{1} & \boldsymbol{x} \\
0 & 0 & 0
\end{array}\right) \mid(t, \boldsymbol{x}, \boldsymbol{y}) \in \mathbb{R}^{2 D+1}\right\},
$$

where $\mathbb{1}$ is the $D \times D$ identity matrix and $\mathfrak{b}$ with the Lie subalgebra

$$
\mathfrak{b}=\left\{\left(\begin{array}{lll}
0 & 0 & \boldsymbol{y} \\
0 & 0 & 0 \\
0 & 0 & 0
\end{array}\right) \mid \boldsymbol{y} \in \mathbb{R}^{D}\right\}
$$

The Lie algebras $\mathfrak{b} \subset \mathfrak{s} \subset \mathfrak{g l}(2 D+1, \mathbb{R})$ are the Lie algebras of the subgroups $\overline{\mathcal{B}} \subset \overline{\mathcal{S}} \subset$ $\mathrm{GL}(2 D+1, \mathbb{R})$ given by

$$
\overline{\mathcal{S}}=\left\{\left(\begin{array}{ccc}
a(t) \mathbb{1} & b(t) \mathbb{1} & \boldsymbol{y} \\
c(t) \mathbb{1} & d(t) \mathbb{1} & \boldsymbol{x} \\
0 & 0 & 1
\end{array}\right) \mid(t, \boldsymbol{x}, \boldsymbol{y}) \in \mathbb{R}^{2 D+1}\right\} \quad \text { and } \quad \overline{\mathcal{B}}=\left\{\left(\begin{array}{lll}
\mathbb{1} & 0 & \boldsymbol{y} \\
0 & \mathbb{1} & 0 \\
0 & 0 & 1
\end{array}\right) \mid \boldsymbol{y} \in \mathbb{R}^{D}\right\}
$$

for some functions $a(t), b(t), c(t), d(t)$ which are given explicitly by

$$
\left(\begin{array}{ll}
a(t) & b(t) \\
c(t) & d(t)
\end{array}\right)=\frac{1}{\gamma-1}\left(\begin{array}{cc}
\gamma e^{t}-e^{\gamma t} & \gamma\left(e^{\gamma t}-e^{t}\right) \\
e^{t}-e^{\gamma t} & \gamma e^{t \gamma}-e^{t}
\end{array}\right)
$$

for $\mathrm{dSG}_{\gamma}$ with $\gamma \in(-1,1)$,

$$
\left(\begin{array}{ll}
a(t) & b(t) \\
c(t) & d(t)
\end{array}\right)=\left(\begin{array}{cc}
e^{t}(1-t) & e^{t} t \\
-e^{t} t & e^{t}(1+t)
\end{array}\right)
$$

for $\mathrm{dSG}_{1}$, and

$$
\left(\begin{array}{ll}
a(t) & b(t) \\
c(t) & d(t)
\end{array}\right)=\left(\begin{array}{cc}
e^{t \chi}(\cos t-\chi \sin t) & e^{t \chi}\left(1+\chi^{2}\right) \sin t \\
-e^{t \chi} \sin t & e^{t \chi}(\cos t+\chi \sin t)
\end{array}\right)
$$

for $\mathrm{AdSG}_{\chi}$ with $\chi>0$. The homogeneous space $\bar{M}=\overline{\mathcal{S}} / \overline{\mathcal{B}}$, if not simply connected, is nevertheless a discrete quotient of the simply-connected $M$ and, as argued at the end of section 3.4, it is enough to show that the orbits of boosts in $\bar{M}$ are generically non-compact to deduce that the same holds for $M$.

Let us denote by $g(t, \boldsymbol{x}, \boldsymbol{y}) \in \overline{\mathcal{S}}$ the generic group element

$$
g(t, \boldsymbol{x}, \boldsymbol{y})=\left(\begin{array}{ccc}
a(t) \mathbb{1} & b(t) \mathbb{1} & \boldsymbol{y} \\
c(t) \mathbb{1} & d(t) \mathbb{1} & \boldsymbol{x} \\
0 & 0 & 1
\end{array}\right) \in \overline{\mathcal{S}}
$$


so that the generic boost is given by

$$
g(0,0, \boldsymbol{y})=\left(\begin{array}{lll}
\mathbb{1} & 0 & \boldsymbol{y} \\
0 & \mathbb{1} & 0 \\
0 & 0 & 1
\end{array}\right) \in \overline{\mathcal{B}}
$$

Parenthetically, let us remark that while it might be tempting to identify $\bar{M}$ with the submanifold of $\overline{\mathcal{S}}$ consisting of matrices of the form $g(t, \boldsymbol{x}, 0)$, this would not be correct. For this to hold true, it would have to be the case that given $g(t, \boldsymbol{x}, \boldsymbol{y})$, there is some $g(0,0, \boldsymbol{w})$ such that $g(t, \boldsymbol{x}, \boldsymbol{y}) g(0,0, \boldsymbol{w})=g\left(t^{\prime}, \boldsymbol{x}^{\prime}, 0\right)$ for some $t^{\prime}, \boldsymbol{x}^{\prime}$. As we now show, this is only ever the case provided that $a(t) \neq 0$. Indeed,

$$
g(t, \boldsymbol{x}, \boldsymbol{y}) g(0,0, \boldsymbol{w})=g(t, c(t) \boldsymbol{w}+\boldsymbol{x}, a(t) \boldsymbol{w}+\boldsymbol{y}),
$$

and hence this is of the form $g\left(t^{\prime}, \boldsymbol{x}^{\prime}, 0\right)$ if and only if we can solve $a(t) \boldsymbol{w}+\boldsymbol{y}=0$ for $\boldsymbol{w}$. Clearly this cannot be done if $a(t)=0$, which may happen for $\mathrm{dSG}_{\gamma \in(0,1)}$ at $t=\frac{\log \gamma}{\gamma-1}$ and for $\operatorname{AdSG}_{\chi>0}$ at $\cos t= \pm \frac{\chi}{\sqrt{1+\chi^{2}}}$.

The action of the boosts on $\bar{M}$ is induced by left multiplication on $\overline{\mathcal{S}}$ :

$$
g(0,0, \boldsymbol{v}) g(t, \boldsymbol{x}, \boldsymbol{y})=g(t, \boldsymbol{x}, \boldsymbol{y}+\boldsymbol{v})
$$

which simply becomes a translation $\boldsymbol{y} \mapsto \boldsymbol{y}+\boldsymbol{v}$ in $\mathbb{R}^{D}$. This is non-compact in $\overline{\mathcal{S}}$, but we need to show that it is non-compact in $\bar{M}$.

The right action of $\overline{\mathcal{B}}$ is given by

$$
g(t, \boldsymbol{x}, \boldsymbol{y}) g(0,0, \boldsymbol{w})=g(t, \boldsymbol{x}+c(t) \boldsymbol{w}, \boldsymbol{y}+a(t) \boldsymbol{w}),
$$

which is again a translation $(\boldsymbol{x}, \boldsymbol{y}) \mapsto(\boldsymbol{x}+c(t) \boldsymbol{w}, \boldsymbol{y}+a(t) \boldsymbol{w})$ in $\mathbb{R}^{2 D}$. The quotient $\mathbb{R}^{2 D} / \overline{\mathcal{B}}$ is the quotient vector space $\mathbb{R}^{2 D} / \mathbb{B}$, where $\mathbb{B} \subset \mathbb{R}^{2 D}$ is the image of the linear map $\mathbb{R}^{D} \rightarrow \mathbb{R}^{2 D}$ sending $\boldsymbol{w} \rightarrow(c(t) \boldsymbol{w}, a(t) \boldsymbol{w})$. Notice that $(a(t), c(t)) \neq(0,0)$ for all $t$, since the matrices in $\overline{\mathcal{S}}$ are invertible, hence $\mathbb{B} \cong \mathbb{R}^{D}$ and hence the quotient vector space $\mathbb{R}^{2 D} / \mathbb{B} \cong \mathbb{R}^{D}$. By the Heine-Borel theorem, it suffices to show that the orbit is unbounded to conclude that it is not compact. Let $[(\boldsymbol{x}, \boldsymbol{y})] \in \mathbb{R}^{2 D} / \mathbb{B}$ denote the equivalence class modulo $\mathbb{B}$ of $(\boldsymbol{x}, \boldsymbol{y}) \in \mathbb{R}^{2 D}$. The distance $d$ between $[(\boldsymbol{x}, \boldsymbol{y})]$ and the boosted $[(\boldsymbol{x}, \boldsymbol{y}+\boldsymbol{v})]$ is the minimum of the distance between $(\boldsymbol{x}, \boldsymbol{y})$ and any point on the coset $[(\boldsymbol{x}, \boldsymbol{y}+\boldsymbol{v})]$; that is,

$$
d=\min _{\boldsymbol{w}}\|(\boldsymbol{x}+c(t) \boldsymbol{w}, \boldsymbol{y}+\boldsymbol{v}+a(t) \boldsymbol{w})-(\boldsymbol{x}, \boldsymbol{y})\|=\min _{\boldsymbol{w}}\|(c(t) \boldsymbol{w}, \boldsymbol{v}+a(t) \boldsymbol{w})\| .
$$

Completing the square, we find

$$
\|(c \boldsymbol{w}, \boldsymbol{v}+a \boldsymbol{w})\|^{2}=\left(a^{2}+c^{2}\right)\left\|\boldsymbol{w}+\frac{a}{a^{2}+c^{2}} \boldsymbol{v}\right\|^{2}+\frac{c^{2}}{a^{2}+c^{2}}\|\boldsymbol{v}\|^{2},
$$

whose minimum occurs when $\boldsymbol{w}=-\frac{a}{a^{2}+c^{2}} \boldsymbol{v}$, resulting in

$$
d=\frac{|c(t)|}{\sqrt{a(t)^{2}+c(t)^{2}}}\|\boldsymbol{v}\| .
$$


As we rescale $\boldsymbol{v} \mapsto s \boldsymbol{v}$, this is unbounded provided that $c(t) \neq 0$. From equations (6.85), (6.86) and (6.87), we see that for $\mathrm{dSG}_{\gamma \in(-1,1]}, c(t)=0$ if and only if $t=0$, whereas for $\mathrm{AdSG}_{\chi>0}, c(t)=0$ if and only if $t=n \pi$ for $n \in \mathbb{Z}$, and hence, in summary, the generic orbits are non compact.

Let us remark that for $\mathrm{AdSG}_{\chi>0}$, if $t=n \pi$ for $n \neq 0$ then the exponential coordinate system breaks down, so that we should restrict to $t \in(-\pi, \pi)$. Indeed, using the explicit matrix representation, one can determine when the exponential coordinates on $\bar{M}$ stop being injective; that is, when there are $(t, \boldsymbol{x})$ and $\left(t^{\prime}, \boldsymbol{x}^{\prime}\right)$ such that $\exp (t H+\boldsymbol{x} \cdot \boldsymbol{P})=$ $\exp \left(t^{\prime} H+\boldsymbol{x}^{\prime} \cdot \boldsymbol{P}\right) B$ for some $B \in \overline{\mathcal{B}}$. In $\mathrm{dSG}_{\gamma \in(-1,1]}$ this only happens when $t=t^{\prime}$ and $\boldsymbol{x}=\boldsymbol{x}^{\prime}$, but in $\mathrm{AdSG}_{\chi>0}$ it happens whenever $t=t^{\prime}=n \pi(n \neq 0)$ and, if so, for all $\boldsymbol{x}, \boldsymbol{x}^{\prime}$.

It now remains to look at the case of spacetime $S 12_{\gamma, \chi}$. This case is very similar to $\mathrm{dSG}_{\gamma}$ in $D=1$ except for two important changes: we work over the complex numbers and $\gamma$ is replaced by $z=\gamma+i \chi$. This means that the (real) subalgebras $\mathfrak{b} \subset \mathfrak{s} \subset \mathfrak{g l}(3, \mathbb{C})$ are given by

$$
\mathfrak{b}=\left\{\left(\begin{array}{lll}
0 & 0 & y \\
0 & 0 & 0 \\
0 & 0 & 0
\end{array}\right) \mid y \in \mathbb{C}\right\} \quad \text { and } \quad \mathfrak{s}=\left\{\left(\begin{array}{ccc}
0 & t z & y \\
-t t(1+z) & x \\
0 & 0 & 0
\end{array}\right) \mid t \in \mathbb{R}, \mathfrak{x}, y \in \mathbb{C}\right\},
$$

whereas the (real) subgroups $\overline{\mathcal{B}} \subset \overline{\mathcal{S}} \subset \mathrm{GL}(3, \mathbb{C})$ are given by

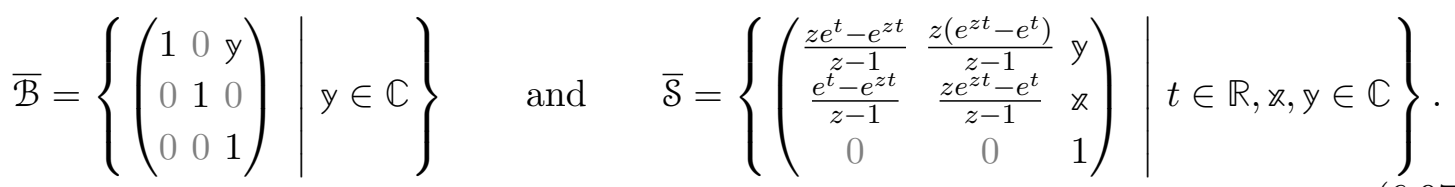

Let $g(t, x, y)$ denote the typical element (shown above) in $\overline{\mathcal{S}}$ and let $g(0,0, y)$ denote the typical element of $\overline{\mathcal{B}}$. Then we have

$$
g(0,0, \boldsymbol{v}) g(t, \mathfrak{x}, \boldsymbol{y})=g(t, x, y+v) \quad \text { and } \quad g(t, x, y) g(0,0, \beta)=g(t, x+a(t) \beta, y+c(t) \beta),
$$

where

$$
a(t)=\frac{z e^{t}-e^{z t}}{z-1} \quad \text { and } \quad c(t)=\frac{e^{t}-e^{z t}}{z-1} .
$$

Hence the left and right action of the boosts takes place in $\mathbb{C}^{2}$ : under the left action $(x, y) \mapsto(x, y+v)$, whereas under the right action $(x, y) \mapsto(x+c(t) \beta, y+a(t) \beta)$.

Now $\mathbb{C}^{2}$ is equivalent to $\mathbb{R}^{4}$ as a metric space and hence the Heine-Borel theorem applies and all we need to show is that the generic orbits are not bounded. The squared distance (in the quotient $\overline{\mathcal{S}} / \overline{\mathcal{B}})$ between a point $[g(t, x, y)]$ and its boost $[g(t, x, y+v)]$ with parameter $\checkmark$ is

$$
\begin{aligned}
\min _{\beta}\|(\mathfrak{x}+c(t) \beta, y+v+a(t) \beta)-(\mathfrak{x}, \mathbf{y})\|^{2} & =\min _{\beta}\|(c(t) \beta, \vee+a(t) \beta)\|^{2} \\
& =\min _{\beta}\left(|c(t)|^{2}|\beta|^{2}+|\vee+a(t) \beta|^{2}\right) .
\end{aligned}
$$

We complete the square and write this as

$$
\min _{\beta}\left(\left(|a|^{2}+|c|^{2}\right)\left|\beta+\frac{\bar{a} \vee}{|a|^{2}+|c|^{2}}\right|^{2}+|\vee|^{2}\left(1-\frac{|a|^{2}}{|a|^{2}+|c|^{2}}\right)\right)=\frac{|c|^{2}|\mathbb{v}|^{2}}{|a|^{2}+|c|^{2}}
$$


where we have used that $a(t)$ and $c(t)$ cannot both be zero because $g(t, x, y)$ is invertible for all $t$. This grows without bound with $\vee$ provided that $c(t) \neq 0$. Since $z \neq 1$, equation (6.99) says that $c(t)=0$ for those $t$ satisfying

$$
e^{z t}=e^{t} \Longleftrightarrow e^{(z-1) t}=1 \Longleftrightarrow(z-1) t=2 \pi i n \quad \exists n \in \mathbb{Z} .
$$

But $z-1=(\gamma-1)+i \xi$ and $\gamma \neq 1$, so that this can only be true for $n=0$ and hence $t=0$. Hence the generic orbit $(t \neq 0)$ is unbounded and hence not compact. Here too one can show that the exponential coordinate system is everywhere valid, by working explicitly with the matrices and checking that the equation $\exp (t H+\boldsymbol{x} \cdot \boldsymbol{P})=\exp \left(t^{\prime} H+\boldsymbol{x}^{\prime} \cdot \boldsymbol{P}\right) B$ for some $B \in \overline{\mathcal{B}}$ has the unique solution $t=t^{\prime}$ and $\boldsymbol{x}=\boldsymbol{x}^{\prime}$ (and hence $B=\mathbb{1}$ ).

\section{Carrollian light cone (LC)}

The carrollian light cone LC is a hypersurface in Minkowski spacetime, identifiable with the future light cone. It does not arise as a limit and has additional brackets $[H, \boldsymbol{B}]=\boldsymbol{B}$, $[H, \boldsymbol{P}]=-\boldsymbol{P}$ and $[\boldsymbol{B}, \boldsymbol{P}]=H+\boldsymbol{J}$, which shows that it is a non-reductive homogeneous spacetime.

\subsection{Action of the boosts}

Although it might be tempting to use that the boosts in Minkowski spacetime act with generic non-compact orbits to deduce the same about the boosts in LC, one has to be careful because what we call boosts in LC might not be interpretable as boosts in the ambient Minkowski spacetime. Indeed, as we will now see, boosts in LC are actually null rotations in the ambient Minkowski spacetime.

We first exhibit the isomorphism between the LC Lie algebra and $\mathfrak{s o}(D+1,1)$. In the LC Lie algebra, the boosts and translations obey the following brackets:

$$
[H, \boldsymbol{B}]=\boldsymbol{B}, \quad[H, \boldsymbol{P}]=-\boldsymbol{P}, \quad \text { and } \quad[\boldsymbol{B}, \boldsymbol{P}]=H+\boldsymbol{J} .
$$

If we let $L_{\mu \nu}$ be the standard generators of $\mathfrak{s o}(D+1,1)$ with $\mu=(0, a, \natural), a=1, \ldots, D$, and with Lie brackets

$$
\left[L_{\mu \nu}, L_{\rho \sigma}\right]=\eta_{\nu \rho} L_{\mu \sigma}-\eta_{\mu \rho} L_{\nu \sigma}-\eta_{\nu \sigma} L_{\mu \rho}+\eta_{\mu \sigma} L_{\nu \rho}
$$

where $\eta_{a b}=\delta_{a b}, \eta_{00}=-1$, and $\eta_{\text {比 }}=1$, then the correspondence is:

$$
J_{a b}=L_{a b}, \quad B_{a}=\frac{1}{\sqrt{2}}\left(L_{0 a}+L_{a \natural}\right), \quad P_{a}=\frac{1}{\sqrt{2}}\left(L_{0 a}-L_{a \natural}\right), \quad \text { and } \quad H=-L_{0 \natural} .
$$

We see that, as advertised, the boosts $B_{a}$ are indeed null rotations.

The boosts act linearly on the ambient coordinates $X^{\mu}$ in Minkowski spacetime, with fundamental vector fields

$$
\zeta_{B_{a}}=\frac{1}{\sqrt{2}}\left(-X^{0} \frac{\partial}{\partial X^{a}}-X^{a} \frac{\partial}{\partial X^{0}}+X^{a} \frac{\partial}{\partial X^{\natural}}-X^{\natural} \frac{\partial}{\partial X^{a}}\right) .
$$


Consider a linear combination $B=w^{a} B_{a}$ and let $T:=X^{0}, X:=w^{a} X^{a}$, and $Y:=X^{\natural}$, so that in terms of these coordinates and dropping the factor of $\frac{1}{\sqrt{2}}$,

$$
\zeta_{B}=-T \frac{\partial}{\partial X}-X \frac{\partial}{\partial T}+X \frac{\partial}{\partial Y}-Y \frac{\partial}{\partial X} .
$$

This allows us to examine the orbit of this vector field while focussing on the threedimensional space with coordinates $T, X, Y$. The vector field is linear, so there is a matrix $A$ such that

$$
\zeta_{B}=\left(\begin{array}{lll}
T & X & Y
\end{array}\right) A\left(\begin{array}{l}
\frac{\partial}{\partial T} \\
\frac{\partial}{\partial X} \\
\frac{\partial}{\partial Y}
\end{array}\right) \Longrightarrow A=\left(\begin{array}{ccc}
0 & -1 & 0 \\
-1 & 0 & 1 \\
0 & -1 & 0
\end{array}\right)
$$

The matrix $A$ obeys $A^{3}=0$, so its exponential is

$$
\exp (s A)=\left(\begin{array}{ccc}
1+\frac{1}{2} s^{2} & -s & -\frac{1}{2} s^{2} \\
-s & 1 & s \\
\frac{1}{2} s^{2} & -s & 1-\frac{1}{2} s^{2}
\end{array}\right)
$$

and hence the orbit of $\left(T_{0}, X_{0}, Y_{0}, \ldots\right)$ is given by

$$
\begin{aligned}
& T(s)=\left(1+\frac{1}{2} s^{2}\right) T_{0}-s X_{0}-\frac{1}{2} s^{2} Y_{0} \\
& X(s)=-s T_{0}+X_{0}+s Y_{0} \\
& Y(s)=\frac{1}{2} s^{2} T_{0}-s X_{0}+\left(1-\frac{1}{2} s^{2}\right) Y_{0},
\end{aligned}
$$

with all other coordinates inert, which is clearly non-compact in the Minkowski spacetime. But of course, this orbit lies on the future light cone (indeed, notice that $-T(s)^{2}+X(s)^{2}+$ $\left.Y(s)^{2}=-T_{0}^{2}+X_{0}^{2}+Y_{0}^{2}\right)$, which is a submanifold, and hence the orbit is also non-compact on LC, provided with the subspace topology.

\subsection{Fundamental vector fields}

Let $A=t H+\boldsymbol{x} \cdot \boldsymbol{P}$ and let us calculate the action of $\operatorname{ad}_{A}$ on the generators, this time with the indices written explicitly:

$$
\begin{aligned}
\operatorname{ad}_{A} B_{a} & =t B_{a}-x^{a} H-x^{b} J_{a b} \\
\operatorname{ad}_{A} P_{a} & =-t P_{a} \\
\operatorname{ad}_{A} H & =x^{a} P_{a} \\
\operatorname{ad}_{A} J_{a b} & =x^{a} P_{b}-x^{b} P_{a} .
\end{aligned}
$$

In order to compute the fundamental vector fields using equation (3.16) and the soldering form using equation (3.45), we need to calculate the action of certain universal power series on $\operatorname{ad}_{A}$ on the generators. To this end, let us derive formulae for the action of $f\left(\operatorname{ad}_{A}\right)$, for $f(z)$ an analytic function of $z$, on the generators. We will do this by first calculating powers of $\operatorname{ad}_{A}$ on generators. It is clear, first of all, that on $\boldsymbol{P}$,

$$
f\left(\operatorname{ad}_{A}\right) \boldsymbol{P}=f(-t) \boldsymbol{P} .
$$


On $H$ and $\boldsymbol{J}$ we just need to treat the constant term separately:

$$
\begin{aligned}
f\left(\operatorname{ad}_{A}\right) H & =f(0) H-\frac{1}{t}(f(-t)-f(0)) \boldsymbol{x} \cdot \boldsymbol{P} \\
f\left(\operatorname{ad}_{A}\right) J_{a b} & =f(0) J_{a b}-\frac{1}{t}(f(-t)-f(0))\left(x^{a} P_{b}-x^{b} P_{a}\right) .
\end{aligned}
$$

On $\boldsymbol{B}$ it is a little bit more complicated. Notice first of all that whereas

$$
\operatorname{ad}_{A}^{2} B_{a}=t \operatorname{ad}_{A} B_{a}-2 x^{a} x^{b} P_{b}+x^{2} P_{a},
$$

$\operatorname{ad}_{A}^{3} B_{a}=t^{2} \operatorname{ad}_{A} B_{a}$. Therefore, by induction, for all $n \geq 1$,

$$
\operatorname{ad}_{A}^{n} B_{a}= \begin{cases}t^{n-1} \operatorname{ad}_{A} B_{a} & n \text { odd } \\ t^{n-1} \operatorname{ad}_{A} B_{a}+t^{n-2}\left(x^{2} P_{a}-2 x^{a} \boldsymbol{x} \cdot \boldsymbol{P}\right) & n \text { even }\end{cases}
$$

and therefore

$$
\begin{aligned}
f\left(\operatorname{ad}_{A}\right) B_{a}= & f(t) B_{a}-\frac{1}{t}(f(t)-f(0))\left(x^{a} H+x^{b} J_{a b}\right) \\
& +\frac{1}{t^{2}}\left(\frac{1}{2}(f(t)+f(-t))-f(0)\right)\left(x^{2} P_{a}-2 x^{a} \boldsymbol{x} \cdot \boldsymbol{P}\right) .
\end{aligned}
$$

Using these formulae, we can now apply equation (3.16) in order to determine the expression of the fundamental vector fields in terms of exponential coordinates.

Let us take $X=\boldsymbol{v} \cdot \boldsymbol{P}$ in equation (3.16). We must take $Y^{\prime}(0)=0$ here and find that

$$
\boldsymbol{y} \cdot \boldsymbol{P}=G\left(\operatorname{ad}_{A}\right) \boldsymbol{v} \cdot \boldsymbol{P}=G(-t) \boldsymbol{v} \cdot \boldsymbol{P} \Longrightarrow \boldsymbol{y}=\frac{t}{1-e^{-t}} \boldsymbol{v},
$$

resulting in

$$
\xi_{P_{a}}=\frac{t}{1-e^{-t}} \frac{\partial}{\partial x^{a}} .
$$

Taking $X=H$ in equation (3.16), we again must take $Y^{\prime}(0)=0$. Doing so, we arrive at

$$
\begin{aligned}
\tau H+\boldsymbol{y} \cdot \boldsymbol{P}=G\left(\operatorname{ad}_{A}\right) H=H- & \frac{1}{t}(G(-t)-1) \boldsymbol{x} \cdot \boldsymbol{P} \\
& \Longrightarrow \tau=1 \quad \text { and } \quad \boldsymbol{y}=\left(\frac{1}{t}-1-\frac{1}{e^{t}-1}\right) \boldsymbol{x}
\end{aligned}
$$

resulting in

$$
\xi_{H}=\frac{\partial}{\partial t}+\left(\frac{1}{t}-1-\frac{1}{e^{t}-1}\right) x^{a} \frac{\partial}{\partial x^{a}} .
$$

One checks already that $\left[\xi_{H}, \xi_{P_{a}}\right]=\xi_{P_{a}}$, as expected.

Finally, put $X=\boldsymbol{v} \cdot \boldsymbol{B}$ in equation (3.16) and hence now $Y^{\prime}(0)=\boldsymbol{\beta} \cdot \boldsymbol{B}+\frac{1}{2} \lambda^{a b} J_{a b}$. Substituting this in equation (3.16) and requiring that the $\mathfrak{h}$-terms vanish, we find

$$
\boldsymbol{\beta}=\frac{G(t)}{F(t)} \boldsymbol{v}=e^{-t} \boldsymbol{v} \quad \text { and } \quad \lambda^{a b}=\frac{1-e^{-t}}{t}\left(v^{a} x^{b}-v^{b} x^{a}\right) .
$$


Comparing the $H$ terms, we see that

$$
\tau=\frac{1-e^{-t}}{t} \boldsymbol{x} \cdot \boldsymbol{v}
$$

whereas the $\boldsymbol{P}$ terms give

$$
\boldsymbol{y}=\frac{1-e^{-t}}{2 t} x^{2} \boldsymbol{v}+\frac{1-t-e^{-t}}{t^{2}} \boldsymbol{x} \cdot \boldsymbol{v} \boldsymbol{x}
$$

resulting in

$$
\xi_{B_{a}}=\frac{1-e^{-t}}{t} x^{a} \frac{\partial}{\partial t}+\frac{1-e^{-t}}{2 t} x^{2} \frac{\partial}{\partial x^{a}}+\frac{1-t-e^{-t}}{t^{2}} x^{a} x^{b} \frac{\partial}{\partial x^{b}} .
$$

One checks that, as expected, $\left[\xi_{H}, \xi_{B_{a}}\right]=-\xi_{B_{a}}$ and that $\left[\xi_{B_{a}}, \xi_{P_{b}}\right]=-\delta_{a b} \xi_{H}-\xi_{J_{a b}}$, where $\xi_{J_{a b}}=x^{b} \frac{\partial}{\partial x^{a}}-x^{a} \frac{\partial}{\partial x^{b}}$.

\subsection{Soldering form and canonical connection}

The soldering form can be calculated from equation (3.45) and projecting the result to $\mathfrak{k} / \mathfrak{h}$ :

$$
\begin{aligned}
\theta & =D\left(\operatorname{ad}_{A}\right)(d t \bar{H}+d \boldsymbol{x} \cdot \overline{\boldsymbol{P}})=d t\left(\bar{H}-\frac{D(-t)-1}{t} \boldsymbol{x} \cdot \overline{\boldsymbol{P}}\right)+D(-t) d \boldsymbol{x} \cdot \overline{\boldsymbol{P}} \\
& =d t \bar{H}+\frac{1+t-e^{t}}{t^{2}} \boldsymbol{x} \cdot \overline{\boldsymbol{P}} d t+\frac{e^{t}-1}{t} d \boldsymbol{x} \cdot \overline{\boldsymbol{P}}
\end{aligned}
$$

It follows from the expression of $\theta$ that it is invertible for all $(t, \boldsymbol{x})$, since $\frac{e^{t}-1}{t} \neq 0$ for all $t \in \mathbb{R}$. Its inverse, the vielbein $E$, has components

$$
E_{\bar{H}}=\frac{\partial}{\partial t}+\left(\frac{1}{t}-\frac{1}{e^{t}-1}\right) x^{a} \frac{\partial}{\partial x^{a}} \quad \text { and } \quad E_{\bar{P}_{a}}=\frac{t}{e^{t}-1} \frac{\partial}{\partial x^{a}} .
$$

The invariant carrollian structure is given by $\kappa=E_{\bar{H}}$ and spatial metric $b=\pi^{2}(\theta, \theta)$, given by

$$
b=\frac{\left(1+t-e^{t}\right)^{2}}{t^{4}} x^{2} d t^{2}+\frac{\left(e^{t}-1\right)^{2}}{t^{2}} d \boldsymbol{x} \cdot d \boldsymbol{x}+2 \frac{\left(e^{t}-1\right)\left(1+t-e^{t}\right)}{t^{3}} \boldsymbol{x} \cdot d \boldsymbol{x} d t
$$

\section{Exotic two-dimensional spacetimes}

In this section, we discuss the two-dimensional homogeneous spacetimes in table 1 . These spacetimes can be treated together. They are reductive, symmetric and even affine, but have no invariant metrics, galilean or carrollian structures. Relative to exponential coordinates $(t, x)$, where $\sigma(t, x)=\exp (t H+x P)$, the soldering form is

$$
\theta=d t H+d x P
$$

and the invariant connection $\omega=0$. The vielbein are

$$
E_{H}=\frac{\partial}{\partial t} \quad \text { and } \quad E_{P}=\frac{\partial}{\partial x} .
$$


The exponential coordinates are affine, so that

$$
\xi_{H}=\frac{\partial}{\partial t} \quad \text { and } \quad \xi_{P}=\frac{\partial}{\partial x} .
$$

The only distinguishing feature is the action of the boosts. We will see that in all cases the fundamental vector field $\xi_{B}$ is linear in the affine coordinates, so we will be able to determine the orbits simply by exponentiating the corresponding matrix. Indeed, we will see that

$$
\xi_{B}=\left(\begin{array}{ll}
t & x
\end{array}\right)\left(\begin{array}{ll}
a & b \\
c & d
\end{array}\right)\left(\begin{array}{l}
\frac{\partial}{\partial t} \\
\frac{\partial}{\partial x}
\end{array}\right)=(a t+c x) \frac{\partial}{\partial t}+(b t+d x) \frac{\partial}{\partial x},
$$

and hence the orbit of the boost through $\left(t_{0}, x_{0}\right)$ is given by

$$
\left(\begin{array}{l}
t(s) \\
x(s)
\end{array}\right)=\exp (s A)\left(\begin{array}{c}
t_{0} \\
x_{0}
\end{array}\right) \quad \text { for } \quad A=\left(\begin{array}{ll}
a & b \\
c & d
\end{array}\right) .
$$

As we saw in section 4.5 , in all cases but $\mathrm{S} 19 \chi$, the only invariant connection is the canonical connection.

\subsection{Spacetime S17}

Here $[B, H]=P$ and $[B, P]=-H-2 P$, so that

$$
\xi_{B}=-x \frac{\partial}{\partial t}-(2 x-t) \frac{\partial}{\partial x}
$$

From equation (8.4), we see that the matrix $A$ in equation (8.5) is given by

$$
A=\left(\begin{array}{cc}
0 & 1 \\
-1 & -2
\end{array}\right) \Longrightarrow \exp (s A)=e^{-s}\left(\begin{array}{cc}
1+s & s \\
-s & 1-s
\end{array}\right) \text {. }
$$

The vector field is complete, and the orbits are homeomorphic to the real line, except for the critical point at the origin which is its own orbit.

\subsection{Spacetime S18}

Here $[B, H]=-H$ and $[B, P]=-P$, so that

$$
\xi_{B}=-t \frac{\partial}{\partial t}-x \frac{\partial}{\partial x}
$$

From equation (8.4), we see that the matrix $A$ in equation (8.5) is given by

$$
A=\left(\begin{array}{cc}
-1 & 0 \\
0 & -1
\end{array}\right) \Longrightarrow \exp (s A)=e^{-s}\left(\begin{array}{ll}
1 & 0 \\
0 & 1
\end{array}\right) .
$$

Again, the vector field is complete, and the orbits are homeomorphic to the real line, except for the critical point at the origin which is its own orbit. 


\subsection{Spacetime $\mathrm{S} 19_{\chi}$}

Here $[B, H]=-(1+\chi) H$ and $[B, P]=(1-\chi) P$, so that

$$
\xi_{B}=-(1+\chi) t \frac{\partial}{\partial t}+(1-\chi) x \frac{\partial}{\partial x} .
$$

From equation (8.4), we see that the matrix $A$ in equation (8.5) is given by

$$
A=\left(\begin{array}{cc}
-(1+\chi) & 0 \\
0 & 1-\chi
\end{array}\right) \Longrightarrow \exp (s A)=\left(\begin{array}{cc}
e^{-s(1+\chi)} & 0 \\
0 & e^{s(1-\chi)}
\end{array}\right) .
$$

Here $\chi>0$. The vector field is complete, and for $\chi \neq 1$ the orbits are homeomorphic to the real line, except for the critical point at the origin which is its own orbit. For $\chi=1$, every point on the $x$-axis $(t=0)$ is its own orbit, but the other orbits are non-compact.

If $\chi=1$, we have a three-parameter family of invariant connections characterised by the Nomizu map in equation (4.54). The torsion and curvature have components

$$
\Theta(H, P)=\left(\nu^{\prime}-\xi^{\prime}\right) H \quad \text { and } \quad \Omega(H, P) P=\nu^{\prime}\left(\zeta^{\prime}-\xi^{\prime}\right) H .
$$

Therefore, there is a two-parameter family of torsion-free invariant connections and two one-parameter families of torsion-free, flat connections:

$$
\alpha(P, P)=\zeta^{\prime} P \quad \text { and } \quad \begin{aligned}
\alpha(H, P) & =\nu^{\prime} H \\
\alpha(P, H) & =\nu^{\prime} H \\
\alpha(P, P) & =\nu^{\prime} P .
\end{aligned}
$$

If $\chi=3$, we have a one-parameter family of invariant connections, which are flat and torsion-free, with Nomizu map given by equation (4.55).

\subsection{Spacetime $\mathrm{S}^{2} 0_{\chi}$}

Here $[B, H]=P$ and $[B, P]=-\left(1+\chi^{2}\right) H-2 \chi P$, so that

$$
\xi_{B}=-\left(1+\chi^{2}\right) x \frac{\partial}{\partial t}+(t-2 \chi x) \frac{\partial}{\partial x} .
$$

From equation (8.4), we see that the matrix $A$ in equation (8.5) is given by

$$
A=\left(\begin{array}{cc}
0 & 1 \\
-\left(1+\chi^{2}\right) & -2 \chi
\end{array}\right) \Longrightarrow \exp (s A)=e^{-\chi s}\left(\begin{array}{cc}
\cos s+\chi \sin s & \sin s \\
-\left(1+\chi^{2}\right) \sin s & \cos s-\chi \sin s
\end{array}\right) \text {. }
$$

The vector field is complete, and for $\chi>0$ the orbits are homeomorphic to the real line, except for the critical point at the origin which is its own orbit. For $\chi=0$, the orbits are circles, as expected since, as seen in figure $3, \mathrm{~S} 20_{\chi=0}=\mathbb{E}$, the euclidean space.

\section{Aristotelian spacetimes}

In this section we introduce coordinates for the aristotelian spacetimes of table 2 and study their geometric properties. 


\subsection{Static spacetime $(\mathrm{S})$}

This is an affine space and the exponential coordinates $(t, \boldsymbol{x})$ are affine, so that

$$
\xi_{H}=\frac{\partial}{\partial t} \quad \text { and } \quad \xi_{P_{a}}=\frac{\partial}{\partial x^{a}} .
$$

Similarly, the soldering form is $\theta=d t H+d \boldsymbol{x} \cdot \boldsymbol{P}$, the canonical invariant connection vanishes, and so does the torsion. The vielbein is

$$
E_{H}=\xi_{H} \quad \text { and } \quad E_{P_{a}}=\xi_{P_{a}} .
$$

\subsection{Torsional static spacetime (TS)}

Here $[H, \boldsymbol{P}]=\boldsymbol{P}$.

\subsubsection{Fundamental vector fields}

Letting $A=t H+\boldsymbol{x} \cdot \boldsymbol{P}$, we find $\operatorname{ad}_{A} H=-\boldsymbol{x} \cdot \boldsymbol{P}$ and $\operatorname{ad}_{A} \boldsymbol{P}=t \boldsymbol{P}$. Therefore, for any analytic function $f$, we conclude that

$$
f\left(\operatorname{ad}_{A}\right) \boldsymbol{P}=f(t) \boldsymbol{P} \quad \text { and } \quad f\left(\operatorname{ad}_{A}\right) H=f(0) H-\frac{1}{t}(f(t)-f(0)) \boldsymbol{x} \cdot \boldsymbol{P} .
$$

Applying this to equation (3.16), we find

$$
\begin{aligned}
\xi_{H} & =\frac{\partial}{\partial t}+\left(\frac{1}{t}-\frac{1}{e^{t}-1}-1\right) x^{a} \frac{\partial}{\partial x^{a}} \\
\xi_{P_{a}} & =\frac{t}{1-e^{-t}} \frac{\partial}{\partial x^{a}}
\end{aligned}
$$

which one can check obey $\left[\xi_{H}, \xi_{P_{a}}\right]=-\xi_{P_{a}}$, as expected.

\subsubsection{Soldering form and canonical connection}

Applying the same formula to equation (3.45), we find that the canonical invariant connection one-form vanishes in this basis and that the soldering form is given by

$$
\theta=d t\left(H+\frac{1}{t}\left(1-\frac{1-e^{-t}}{t}\right) \boldsymbol{x} \cdot \boldsymbol{P}\right)+\frac{1-e^{-t}}{t} d \boldsymbol{x} \cdot \boldsymbol{P},
$$

so that the corresponding vielbein is

$$
E_{H}=\frac{\partial}{\partial t}+\left(\frac{1}{t}-\frac{1}{1-e^{-t}}\right) x^{a} \frac{\partial}{\partial x^{a}} \quad \text { and } \quad E_{P_{a}}=\frac{t}{1-e^{-t}} \frac{\partial}{\partial x^{a}} .
$$

It is clear from the fact that the function $\frac{1-e^{-t}}{t}$ is never zero that $\theta$ is invertible for all $(t, \boldsymbol{x})$.

Although the canonical connection is flat, its torsion 2-form does not vanish:

$$
\Theta=\frac{e^{-t}-1}{t} d t \wedge d \boldsymbol{x} \cdot \boldsymbol{P}
$$




\subsection{Aristotelian spacetime $\mathrm{A}_{2} 3_{\varepsilon}$}

Here $\left[P_{a}, P_{b}\right]=-\varepsilon J_{a b}$, where $D \geq 2$.

\subsubsection{Fundamental vector fields}

Let $A=t H+\boldsymbol{x} \cdot \boldsymbol{P}$. Then $\operatorname{ad}_{A} H=0$ and $\operatorname{ad}_{A} P_{b}=-\varepsilon x^{a} J_{a b}$. Continuing, we find

$$
\operatorname{ad}_{A}^{2} P_{b}=\varepsilon x^{b} \boldsymbol{x} \cdot \boldsymbol{P}-\varepsilon x^{2} P_{b} \quad \text { and } \quad \operatorname{ad}_{A}^{3} P_{b}=\left(-\varepsilon x^{2}\right) \operatorname{ad}_{A} P_{b} .
$$

Therefore, an induction argument shows that

$$
\operatorname{ad}_{A}^{n} P_{b}=\left(-\varepsilon x^{2}\right) \operatorname{ad}_{A}^{n-2} P_{b} \quad \forall n \geq 3 .
$$

If $f(z)$ is analytic in $z$, then $f\left(\operatorname{ad}_{A}\right) H=f(0) H$ and

$$
\begin{aligned}
f\left(\operatorname{ad}_{A}\right) P_{b}= & \frac{1}{2}\left(f\left(x_{+}\right)+f\left(x_{-}\right)\right) P_{b}-\frac{1}{2}\left(f\left(x_{+}\right)+f\left(x_{-}\right)-2 f(0)\right) \frac{x^{b} \boldsymbol{x} \cdot \boldsymbol{P}}{x^{2}} \\
& -\frac{\varepsilon}{2 x_{+}}\left(f\left(x_{+}\right)-f\left(x_{-}\right)\right) x^{a} J_{a b},
\end{aligned}
$$

where

$$
x_{ \pm}= \pm \sqrt{-\varepsilon x^{2}}= \begin{cases} \pm|x| & \varepsilon=-1 \\ \pm i|\boldsymbol{x}| & \varepsilon=1\end{cases}
$$

Similarly, $\operatorname{ad}_{A} J_{a b}=x^{a} P_{b}-x^{b} P_{a}$, so that

$$
\begin{aligned}
f\left(\operatorname{ad}_{A}\right) J_{a b}= & f(0) J_{a b}+\frac{1}{2}\left(\widetilde{f}\left(x_{+}\right)+\widetilde{f}\left(x_{-}\right)\right)\left(x^{a} P_{b}-x^{b} P_{a}\right) \\
& -\frac{\varepsilon}{2 x_{+}}\left(\widetilde{f}\left(x_{+}\right)-\widetilde{f}\left(x_{-}\right)\right) x^{c}\left(x^{a} J_{c b}-x^{b} J_{c a}\right),
\end{aligned}
$$

where $\widetilde{f}(z)=(f(z)-f(0)) / z$.

Inserting these formulae in equation (3.16) with $X=H$ and $Y^{\prime}(0)=0$, we see that

$$
\xi_{H}=\frac{\partial}{\partial t} \text {. }
$$

If instead $X=\boldsymbol{v} \cdot \boldsymbol{P}$ and $Y^{\prime}(0)=\frac{1}{2} \lambda^{a b} J_{a b}$, we see first of all that $\tau=0$ and that demanding that the $J_{a b}$ terms cancel,

$$
\lambda^{a b}=\frac{-\varepsilon\left(G\left(x_{+}\right)-G\left(x_{-}\right)\right)}{x_{+}\left(F\left(x_{+}\right)+F\left(x_{-}\right)\right)}\left(x^{a} v^{b}-x^{b} v^{a}\right),
$$

and reinserting into equation (3.16), we find that

$$
\begin{aligned}
y^{a}= & \frac{1}{2}\left(G\left(x_{+}\right)+G\left(x_{-}\right)-\frac{\left(G\left(x_{+}\right)-G\left(x_{-}\right)\right)\left(F\left(x_{+}\right)-F\left(x_{-}\right)\right)}{F\left(x_{+}\right)+F\left(x_{-}\right)}\right) v^{a} \\
& -\frac{1}{2}\left(G\left(x_{+}\right)+G\left(x_{-}\right)-2-\frac{\left(G\left(x_{+}\right)-G\left(x_{-}\right)\right)\left(F\left(x_{+}\right)-F\left(x_{-}\right)\right)}{F\left(x_{+}\right)+F\left(x_{-}\right)}\right) \frac{\boldsymbol{v} \cdot \boldsymbol{x}}{x^{2}} x^{a} .
\end{aligned}
$$

From this we read off the expression for $\xi_{P_{a}}$ :

$$
\xi_{P_{a}}=\frac{F\left(x_{+}\right) G\left(x_{-}\right)+F\left(x_{-}\right) G\left(x_{+}\right)}{F\left(x_{+}\right)+F\left(x_{-}\right)} \frac{\partial}{\partial x^{a}}+\left(1-\frac{F\left(x_{+}\right) G\left(x_{-}\right)+F\left(x_{-}\right) G\left(x_{+}\right)}{F\left(x_{+}\right)+F\left(x_{-}\right)}\right) \frac{x^{a} x^{b}}{x^{2}} \frac{\partial}{\partial x^{b}},
$$


which simplifies to

$$
\begin{aligned}
\xi_{P_{a}}^{(\varepsilon=1)} & =|\boldsymbol{x}| \cot |\boldsymbol{x}| \frac{\partial}{\partial x^{a}}+(1-|\boldsymbol{x}| \cot |\boldsymbol{x}|) \frac{x^{a} x^{b}}{x^{2}} \frac{\partial}{\partial x^{b}} \\
\xi_{P_{a}}^{(\varepsilon=-1)} & =|\boldsymbol{x}| \operatorname{coth}|\boldsymbol{x}| \frac{\partial}{\partial x^{a}}+(1-|\boldsymbol{x}| \operatorname{coth}|\boldsymbol{x}|) \frac{x^{a} x^{b}}{x^{2}} \frac{\partial}{\partial x^{b}} .
\end{aligned}
$$

\subsubsection{Soldering form and canonical connection}

The soldering form and connection one-form for the canonical connection are obtained from equation (3.45), which says that

$$
\begin{aligned}
\theta+\omega= & d t H+d x^{b} D\left(\operatorname{ad}_{A}\right) P_{b} \\
= & d t H+\frac{1}{2}\left(D\left(x_{+}\right)+D\left(x_{-}\right)\right) d \boldsymbol{x} \cdot \boldsymbol{P} \\
& -\frac{1}{2}\left(D\left(x_{+}\right) D\left(x_{-}\right)-2\right) \frac{\boldsymbol{x} \cdot d \boldsymbol{x}}{x^{2}} \boldsymbol{x} \cdot \boldsymbol{P}-\frac{\varepsilon}{2 x_{+}}\left(D\left(x_{+}\right)-D\left(x_{-}\right)\right) x^{a} d x^{b} J_{a b},
\end{aligned}
$$

whence

$$
\begin{gathered}
\theta^{(\varepsilon=1)}=d t H+\frac{\sin |\boldsymbol{x}|}{|\boldsymbol{x}|} d \boldsymbol{x} \cdot \boldsymbol{P}+\left(1-\frac{\sin |\boldsymbol{x}|}{|\boldsymbol{x}|}\right) \frac{\boldsymbol{x} \cdot d \boldsymbol{x}}{x^{2}} \boldsymbol{x} \cdot \boldsymbol{P} \\
\theta^{(\varepsilon=-1)}=d t H+\frac{\sinh |\boldsymbol{x}|}{|\boldsymbol{x}|} d \boldsymbol{x} \cdot \boldsymbol{P}+\left(1-\frac{\sinh |\boldsymbol{x}|}{|\boldsymbol{x}|}\right) \frac{\boldsymbol{x} \cdot d \boldsymbol{x}}{x^{2}} \boldsymbol{x} \cdot \boldsymbol{P}
\end{gathered}
$$

and

$$
\begin{aligned}
\omega^{(\varepsilon=1)} & =\frac{1-\cos |\boldsymbol{x}|}{x^{2}} x^{a} d x^{b} J_{a b} \\
\omega^{(\varepsilon=-1)} & =\frac{1-\cosh |\boldsymbol{x}|}{x^{2}} x^{a} d x^{b} J_{a b} .
\end{aligned}
$$

It follows that if $\varepsilon=-1$ the soldering form is invertible for all $(t, \boldsymbol{x})$, whereas if $\varepsilon=1$ then it is invertible for all $t$ but inside the open ball $|\boldsymbol{x}|<\pi$.

The torsion of the canonical connection vanishes, since $[\theta, \theta]_{\mathfrak{m}}=0$. The curvature is given by

$$
\begin{aligned}
\Omega^{(\varepsilon=1)} & =\frac{1}{2} \frac{\sin ^{2}|\boldsymbol{x}|}{x^{2}} d x^{a} \wedge d x^{b} J_{a b}+\frac{\sin |\boldsymbol{x}|}{|x|}\left(1-\frac{\sin |\boldsymbol{x}|}{|x|}\right) \frac{x^{b} x^{c}}{x^{2}} d x^{a} \wedge d x^{c} J_{a b} \\
\Omega^{(\varepsilon=-1)} & =-\frac{1}{2} \frac{\sinh ^{2}|\boldsymbol{x}|}{x^{2}} d x^{a} \wedge d x^{b} J_{a b}-\frac{\sinh |\boldsymbol{x}|}{|x|}\left(1-\frac{\sinh |\boldsymbol{x}|}{|x|}\right) \frac{x^{b} x^{c}}{x^{2}} d x^{a} \wedge d x^{c} J_{a b} .
\end{aligned}
$$

\subsection{Aristotelian spacetime A24}

Here $D=2$ and $\left[P_{a}, P_{b}\right]=\epsilon_{a b} H$.

\subsubsection{Fundamental vector fields}

Letting $A=t H+\boldsymbol{x} \cdot \boldsymbol{P}$, we have that $\operatorname{ad}_{A} H=0$ and $\operatorname{ad}_{A} P_{a}=-\epsilon_{a b} x^{b} H$, whence $\operatorname{ad}_{A}^{2} P_{a}=0$. So if $f(z)$ is analytic in $z$,

$$
f\left(\operatorname{ad}_{A}\right) H=f(0) H \quad \text { and } \quad f\left(\operatorname{ad}_{A}\right) P_{a}=f(0) P_{a}-f^{\prime}(0) \epsilon_{a b} x^{b} H .
$$


Since $G(z)=1-\frac{1}{2} z+O\left(z^{2}\right)$, from equation (3.16) we see that

$$
\xi_{H}=\frac{\partial}{\partial t} \quad \text { and } \quad \xi_{P_{a}}=\frac{\partial}{\partial x^{a}}+\frac{1}{2} \epsilon_{a b} x^{b} \frac{\partial}{\partial t} .
$$

One checks that $\left[\xi_{P_{a}}, \xi_{P_{b}}\right]=-\epsilon_{a b} \xi_{H}$, as expected.

\subsubsection{Soldering form and canonical connection}

Since $D(z)=1-\frac{1}{2} z+O\left(z^{2}\right)$, equation (3.45) says that the connection one-form $\omega=0$ and the soldering form is given by

$$
\theta=\left(d t+\frac{1}{2} \epsilon_{a b} d x^{a} x^{b}\right) H+d \boldsymbol{x} \cdot \boldsymbol{P},
$$

which is clearly everywhere invertible. The torsion of the canonical connection is given by

$$
\Theta=-\frac{1}{2} \epsilon_{a b} d x^{a} \wedge d x^{b} H
$$

The vielbein is given by

$$
E_{H}=\frac{\partial}{\partial t} \quad \text { and } \quad E_{P_{a}}=\frac{\partial}{\partial x^{a}}-\frac{1}{2} \epsilon_{a b} x^{b} \frac{\partial}{\partial t} .
$$

\section{Symmetries of the spacetime structure}

In this section we investigate the (conformal) symmetries of the carrollian and galilean spacetimes and their respective invariant structures. A carrollian structure $(\kappa, b)$ consists of a spatial metric $b$ and a so-called carrollian vector field $\kappa$, whereas a galilean structure $(\tau, h)$ consists of a spatial co-metric $h$ and a clock-one form $\tau$. Let us remark that some authors would add the invariant connection as part of the structure, but we will not do so in the following. This means that, in the terminology of [29], we treat the "weak" rather than the "strong" structures.

The calculations in this section are motivated by the intriguing connection between conformally carrollian symmetries [3, 29] and the symmetries of asymptotic flat spacetimes $[35,36]$ in $3+1$ dimensions. This connection is given by an isomorphism between the Lie algebra of infinitesimal conformal transformations of a carrollian structure [3] and the Lie algebra of the Bondi-Metzner-Sachs (BMS) group [35, 36].

Similarly, the infinitesimal conformal symmetries of the galilean and carrollian structures of the homogeneous kinematical spacetimes will turn out to be infinite-dimensional and one might hope this has interesting consequences. It should be mentioned that were one to add the invariant connection as part of the data of the homogeneous carrollian or galilean structure, the symmetry algebra would be typically cut down to the (finite-dimensional) transitive kinematical Lie algebra.

Let $(M, \tau, h)$ be a galilean spacetime. We say that a vector field $\xi \in \mathscr{X}(M)$ is a galilean Killing vector field if it generates a symmetry of the galilean structure:

$$
\mathcal{L}_{\xi} \tau=0 \quad \text { and } \quad \mathcal{L}_{\xi} h=0,
$$


whereas we say that it is a galilean conformal Killing vector field at level $N \in \mathbb{N}$ if it generates a conformal symmetry (at level $N$ ) of the galilean structure:

$$
\mathcal{L}_{\xi} \tau=-\frac{\lambda}{N} \tau \quad \text { and } \quad \mathcal{L}_{\xi} h=\lambda h,
$$

for some $\lambda \in C^{\infty}(M)$. Similarly, if $(M, \kappa, b)$ is a carrollian spacetime, we say that $\xi \in$ $\mathscr{X}(M)$ is a carrollian Killing vector field if it generates a symmetry of the carrollian structure:

$$
\mathcal{L}_{\xi} \kappa=0 \quad \text { and } \quad \mathcal{L}_{\xi} b=0,
$$

whereas we say that it is a carrollian conformal Killing vector field at level $N \in \mathbb{N}$ if it generates a conformal symmetry (at level $N$ ) of the carrollian structure:

$$
\mathcal{L}_{\xi} \kappa=-\frac{\lambda}{N} \kappa \quad \text { and } \quad \mathcal{L}_{\xi} b=\lambda b
$$

for some $\lambda \in C^{\infty}(M)$. These definitions agree (modulo notation) with the ones in [37] and [3, 29]. The set of galilean/carrollian Killing vector fields close under the Lie bracket of vector fields to give rise to Lie algebras. The same is true for the set of galilean/carrollian conformal Killing vector fields of a given fixed level $N$. In this section we will determine the structure of these Lie algebras for the homogeneous carrollian and galilean spacetimes.

The calculations in this section are easier to perform if we change coordinates from the exponential coordinates $\sigma: \mathbb{R}^{D+1} \rightarrow M$, with $\sigma(t, \boldsymbol{x})=\exp (t H+\boldsymbol{x} \cdot \boldsymbol{P}) \cdot o$, that we have been using until now to modified exponential coordinates $\sigma^{\prime}: \mathbb{R}^{D+1} \rightarrow M$, with $\sigma^{\prime}(t, \boldsymbol{x})=\exp (t H) \exp (\boldsymbol{x} \cdot \boldsymbol{P}) \cdot o$. Appendix A discusses these coordinates further. In many of the calculations we require knowledge of the Lie algebra of conformal Killing vector fields on the simply-connected riemannian symmetric spaces $\mathbb{E}, \mathbb{S}$ and $\mathbb{H}$. In appendix $\mathrm{B}$ we collect a few standard results in low dimension.

\subsection{Symmetries of the carrollian structure (C)}

We start by determining the carrollian Killing vector fields for the (flat) carrollian spacetime C (as has already been done in, e.g., [3]). Since $H$ and $\boldsymbol{P}$ commute in this spacetime, the exponential and modified exponential coordinates agree. The invariant carrollian structure on the spacetime parametrised by $\left(t, x^{a}\right) \in \mathbb{R}^{D+1}$, with $a=1, \ldots, D$, is given by $\kappa=\frac{\partial}{\partial t}$ and $b=\delta_{a b} d x^{a} d x^{b}$. Let $\xi=\xi^{0} \frac{\partial}{\partial t}+\xi^{a} \frac{\partial}{\partial x^{a}}$ be a carrollian Killing vector field of $(\kappa, b)$, so that it satisfies equation (10.3). Then, $\mathcal{L}_{\xi} \kappa=[\xi, \kappa]=0$ says that $T:=\xi^{0}$ and $\xi^{a}$ are $t$-independent. The condition $\mathcal{L}_{\xi} b=0$, says that

$$
0 \stackrel{!}{=} \mathcal{L}_{\xi} b=2\left(\mathcal{L}_{\xi} d x^{a}\right) d x^{a}=2 d\left(\mathcal{L}_{\xi} x^{a}\right) d x^{a}=2 d \xi^{a} d x^{a}=2 \frac{\partial \xi^{a}}{\partial x^{b}} d x^{b} d x^{a} \Longrightarrow \frac{\partial \xi^{a}}{\partial x^{b}}+\frac{\partial \xi^{b}}{\partial x^{a}}=0 .
$$

This says that $\xi^{a}(\boldsymbol{x}) \frac{\partial}{\partial x^{a}}$ is a Killing vector field of euclidean space. In summary, the most general carrollian Killing vector field of $(\kappa, b)$ is given by

$$
\xi=T(\boldsymbol{x}) \frac{\partial}{\partial t}+\xi_{X}
$$


for some $X \in \mathfrak{e}$, the euclidean Lie algebra of $\mathbb{E}^{D}$, and some "supertranslations" $T \in$ $C^{\infty}\left(\mathbb{E}^{D}\right)$. As a vector space, then, the Lie algebra $\mathfrak{a}$ of carrollian Killing vector fields is given by $C^{\infty}\left(\mathbb{E}^{D}\right) \oplus \mathfrak{e}$, but as a Lie algebra it is a semidirect product

$$
\mathfrak{a}^{\mathrm{C}} \cong \mathfrak{e} \ltimes C^{\infty}\left(\mathbb{E}^{D}\right),
$$

where the action of $\mathfrak{e}$ on $C^{\infty}\left(\mathbb{E}^{D}\right)$ is via the Lie derivative. In other words, we have a split exact sequence

$$
0 \longrightarrow C^{\infty}\left(\mathbb{E}^{D}\right) \longrightarrow \mathfrak{a}^{\mathfrak{C}} \longrightarrow \mathfrak{e} \longrightarrow 0 .
$$

The carrollian algebra is embedded here by considering the subalgebra of $C^{\infty}\left(\mathbb{E}^{D}\right)$ consisting of polynomial functions of degree at most 1: with the constant function 1 corresponding to $H$ and the linear function $x^{a}$ corresponding to $B_{a}$. When we identify $J_{a b}$ and $P_{a}$ in $\mathfrak{e}$ in the obvious way we recover (5.52).

Let us now determine the carrollian conformal Killing vector fields. Let $\xi=\xi^{0} \frac{\partial}{\partial t}+$ $\xi^{a} \frac{\partial}{\partial x^{a}}$ satisfy equation (10.4) where $(\kappa, b)$ is again the invariant carrollian structure on C: $\kappa=\frac{\partial}{\partial t}$ and $b=\delta_{a b} d x^{a} d x^{b}$. The condition $\mathcal{L}_{\xi} \kappa=-\frac{\lambda}{N} \kappa$ imposes

$$
\frac{\partial \xi^{a}}{\partial t}=0 \quad \text { and } \quad \lambda=N \frac{\partial \xi^{0}}{\partial t} .
$$

The condition $\mathcal{L}_{\xi} b=\lambda b$ says that

$$
\frac{\partial \xi^{a}}{\partial x^{b}}+\frac{\partial \xi^{b}}{\partial x^{a}}=\lambda \delta_{a b}
$$

so that $\xi^{a} \frac{\partial}{\partial x^{a}}$ is a conformal Killing vector of $\mathbb{E}^{D}$. Since $\xi^{a}$ is independent of time, so is $\lambda=\frac{2}{D} \frac{\partial \xi^{a}}{\partial x^{a}}$, which we can now use to solve for $\xi^{0}$ in (10.9):

$$
\xi^{0}=T(\boldsymbol{x})+\frac{2 t}{N D} \frac{\partial \xi^{a}}{\partial x^{a}},
$$

for some "supertranslations" $T \in C^{\infty}\left(\mathbb{E}^{D}\right)$. The carrollian conformal symmetries vary with respect to the space dimension $D$.

Let $D \geq 3$. Thus we see that, as a vector space, the Lie algebra $\mathfrak{c}^{\mathrm{C}}$ of carrollian conformal Killing vector fields of $\mathcal{C}$ is isomorphic to $\mathfrak{s o}(D+1,1) \oplus C^{\infty}\left(\mathbb{E}^{D}\right)$, where $\mathfrak{s o}(D+1,1)$ is the Lie algebra of conformal Killing vectors on $\mathbb{E}^{D}$ which we denote by $\xi_{X}$. In summary we have the vector field

$$
\xi=\xi_{X}+\frac{2 t}{N D} \operatorname{div} \xi_{X} \frac{\partial}{\partial t}+T(\boldsymbol{x}) \frac{\partial}{\partial t} .
$$

for $X \in \mathfrak{s o}(D+1,1)$ and $T \in C^{\infty}\left(\mathbb{E}^{D}\right)$, and $\operatorname{div} \xi=\frac{\partial \xi^{a}}{\partial x^{a}}$. The vector space isomorphism is then given by

$$
X \mapsto \xi_{X}+\frac{2 t}{N D} \operatorname{div} \xi_{X} \frac{\partial}{\partial t} \quad \text { and } \quad T \mapsto T \frac{\partial}{\partial t} .
$$

As Lie algebras, $\mathfrak{c}^{\mathrm{C}}$ is a semidirect product. Indeed,

$$
\left[\xi_{X}+\frac{2 t}{N D} \operatorname{div} \xi_{X} \frac{\partial}{\partial t}, T \frac{\partial}{\partial t}\right]=\left(\xi_{X}(T)-\frac{2}{N D} \operatorname{div} \xi_{X} T\right) \frac{\partial}{\partial t},
$$


so that $T$ does not actually transform as a function but as a section of $\mathscr{L}^{\frac{2}{N}}$ where $\mathscr{L}$ is the density line bundle, normalised so that the spatial metric $b$ is a section of $S^{2} T^{*} M \otimes \mathscr{L}^{2}$.

It may help to spell this out. A conformal metric is a section of $S^{2} T^{*} M \otimes \mathscr{L}^{2}$ and a conformal Killing vector field is one which preserves the conformal metric. Now if $\zeta$ is a conformal Killing vector field for $(M, g)$, then

$$
\mathcal{L}_{\zeta} g=\frac{2}{D} \operatorname{div} \zeta g \Longleftrightarrow\left(\mathcal{L}_{\zeta}-\frac{2}{D} \operatorname{div} \zeta\right) g=0
$$

If we interpret this as the invariance of $g$ under the action of $\zeta$ on sections of $S^{2} T^{*} M \otimes \mathscr{L}^{2}$, we see that the action of $\xi_{X}$ on $T$, which is given in equation (10.14) by

$$
T \mapsto\left(\mathcal{L}_{\xi_{X}}-\frac{2}{N D} \operatorname{div} \xi_{X}\right) T
$$

says that $T$ is a section of $\mathscr{L}^{\frac{2}{N}}$, as claimed. In particular, if $N=2, T$ has conformal weight 1 in agreement with [5].

In summary, for $D \geq 3, \mathfrak{c}^{\mathrm{C}}$ is isomorphic to a split extension

$$
0 \longrightarrow \Gamma\left(\mathscr{L}^{\frac{2}{N}}\right) \longrightarrow \mathfrak{c}_{D \geq 3}^{\mathrm{C}} \longrightarrow \mathfrak{s o}(D+1,1) \longrightarrow 0,
$$

a result first derived in [3]. We notice that comparing to the Lie algebra of carrollian Killing vector fields in equation (10.8), all that has happened is that the Lie algebra $\mathfrak{e}$ of euclidean isometries gets enhanced to the Lie algebra $\mathfrak{s o}(D+1,1)$ of euclidean conformal symmetries, under which the "supertranslations" transform not as functions, but as sections of a (trivial) line bundle with conformal weight $2 / N$ (in conventions where the metric scales with weight 2 ). We did not see this when we calculated the carrollian Killing vector fields because the Lie algebra $\mathfrak{e}$ does not contain the generator of dilatations and cannot tell the weight.

Now let $D=2$. In this case, as reviewed in appendix B, the Lie algebra of conformal Killing vector fields on $\mathbb{E}^{2}$ is enhanced to the Lie algebra $\mathscr{O}(\mathbb{C})$ of entire functions on the complex plane with the wronskian Lie bracket: $[f, g]=f \partial g-g \partial f$. Hence for $D=2, \mathfrak{c}^{\mathrm{C}}$ is isomorphic to a split extension

$$
0 \longrightarrow \Gamma\left(\mathscr{L}^{\frac{2}{N}}\right) \longrightarrow \mathfrak{c}_{D=2}^{\complement} \longrightarrow \mathscr{O}(\mathbb{C}) \longrightarrow 0 .
$$

The vector field is given explicitly by

$$
\xi=\xi_{f}+\frac{t}{N} \operatorname{div} \xi_{f} \frac{\partial}{\partial t}+T(z) \frac{\partial}{\partial t} \quad \text { where } \quad \xi_{f}=f(z) \partial+\overline{f(z)} \bar{\partial} .
$$

Finally, if $D=1$, every vector field on $\mathbb{E}^{1}$ is conformal Killing and hence now $\mathfrak{c}^{\mathrm{C}}$ is isomorphic to

$$
0 \longrightarrow \Gamma\left(\mathscr{L}^{\frac{2}{N}}\right) \longrightarrow \mathfrak{c}_{D=1}^{\mathrm{C}} \longrightarrow C^{\infty}(\mathbb{R}) \longrightarrow 0,
$$

where the vector field is given by

$$
\xi=\xi(x) \frac{\partial}{\partial x}+\frac{2 t}{N} \xi^{\prime}(x) \frac{\partial}{\partial t}+T(x) \frac{\partial}{\partial t} .
$$

The last two results were already obtained in section IV of [29], to which we refer for further information. 


\subsection{Symmetries of the (anti) de Sitter carrollian structure (dSC and AdSC)}

We now investigate the symmetries of the (anti) de Sitter carrollian spacetimes (dSC and AdSC) with their carrollian structure. They can be embedded as null surfaces of the (anti) de Sitter spacetime. Unlike the carrollian space C, the invariant connection on these spacetimes is not flat. The carrollian structure becomes much more transparent if we work in modified exponential coordinates, as described in appendix A. In order to be able to treat both cases at once, let us introduce the functions

$$
C(r):=\left\{\begin{array}{ll}
\cos (r) & \text { for dSC } \\
\cosh (r) & \text { for AdSC }
\end{array} \quad S(r):=C^{\prime}(r) \quad \text { and } \quad G(r):=\frac{S(r)}{C(r)},\right.
$$

with the understanding that $r \in\left(0, \frac{\pi}{2}\right)$ for $\mathrm{dSC}$ and $r>0$ for AdSC. In those coordinates, the invariant carrollian structures are given by

$$
\kappa=C(r)^{-1} \frac{\partial}{\partial t} \quad \text { and } \quad b=d r^{2}+S(r)^{2} g_{\mathbb{S}^{D-1}} .
$$

The metric $b$ defines the round metric on the sphere $\mathbb{S}^{D}$ for dSC and the hyperbolic metric on $\mathbb{H}^{D}$ for AdSC. Although the coordinates only cover a hemisphere of $\mathbb{S}^{D}$, we proved in $[6$, $\S$ 4.2.5] that dSC is diffeomorphic to $\mathbb{R} \times \mathbb{S}^{D}$ for $D \geq 2$ and to $\mathbb{R}^{2}$ for $D=1$.

Now let $\xi=\xi^{0} \frac{\partial}{\partial t}+\xi^{a} \frac{\partial}{\partial x^{a}}$ be a carrollian Killing vector field, so that $\mathcal{L}_{\xi} \kappa=0$ and $\mathcal{L}_{\xi} b=0$. We calculate

$$
[\xi, \kappa]=-C(r)^{-1}\left(\left(\frac{\partial \xi^{0}}{\partial t}+\boldsymbol{x} \cdot \boldsymbol{\xi} \frac{G(r)}{r}\right) \frac{\partial}{\partial t}+\frac{\partial \xi^{a}}{\partial t} \frac{\partial}{\partial x^{a}}\right) \stackrel{?}{=} 0
$$

which is solved by

$$
\xi^{a}=\xi^{a}(\boldsymbol{x}) \quad \text { and } \quad \xi^{0}=T(\boldsymbol{x})-t \boldsymbol{x} \cdot \boldsymbol{\xi} \frac{G(r)}{r},
$$

for some $t$ independent "supertranslations" $T(\boldsymbol{x})$ and where we have introduced the shorthand notation $\boldsymbol{x} \cdot \boldsymbol{\xi}=\delta_{a b} x^{a} \xi^{b}$. Therefore,

$$
\xi=\left(T(\boldsymbol{x})-\frac{G(r)}{r} t \boldsymbol{x} \cdot \boldsymbol{\xi}\right) \frac{\partial}{\partial t}+\xi^{a}(\boldsymbol{x}) \frac{\partial}{\partial x^{a}} .
$$

Now we impose $\mathcal{L}_{\xi} b=0$. We observe that this does not constrain the $\frac{\partial}{\partial t}$ component of $\xi$, so it is only a condition on $\xi^{a}(\boldsymbol{x}) \frac{\partial}{\partial x^{a}}$. But in the submanifolds of constant $t, b$ defines a metric and $\mathcal{L}_{\xi} b=0$ says that $\xi^{a}(\boldsymbol{x}) \frac{\partial}{\partial x^{a}}$ is a Killing vector. Therefore, we have

$$
\xi=\left(T(\boldsymbol{x})-\frac{G(r)}{r} t \boldsymbol{x} \cdot \boldsymbol{\xi}_{X}\right) \frac{\partial}{\partial t}+\xi_{X}^{a}(\boldsymbol{x}) \frac{\partial}{\partial x^{a}} \quad \text { for } X \in \begin{cases}\mathfrak{s o}(D+1), & \text { for dSC } \\ \mathfrak{s o}(D, 1), & \text { for AdSC }\end{cases}
$$

In summary, the Lie algebra of carrollian Killing vector fields is isomorphic to

$$
\mathfrak{a}^{\mathrm{dSC}} \cong \mathfrak{s o}(D+1) \ltimes C^{\infty}\left(\mathbb{S}^{D}\right) \quad \text { and } \quad \mathfrak{a}^{\mathrm{AdSC}} \cong \mathfrak{s o}(D, 1) \ltimes C^{\infty}\left(\mathbb{H}^{D}\right),
$$


where the action of $\mathfrak{s o}$ on $C^{\infty}$ is given by

$$
[X, T]=\xi_{X} T+\frac{G(r)}{r} \boldsymbol{x} \cdot \boldsymbol{\xi}_{X} T .
$$

If we define $T \mapsto \widehat{T}:=-C(r) T$ then it follows that

$$
\widehat{[X, T]}=\xi_{X} \widehat{T}
$$

so the action of $\mathfrak{s o}$ on $C^{\infty}$ is just a "dressed" version of the standard action of vector fields on functions. ${ }^{11}$ In this way, we may identify the finite-dimensional transitive kinematical Lie algebras as the subalgebras

$$
\mathfrak{s o}(D+1) \ltimes C_{\leq 1}^{\infty}\left(\mathbb{S}^{D}\right) \quad \text { and } \quad \mathfrak{s o}(D, 1) \ltimes C_{\leq 1}^{\infty}\left(\Re^{D}\right),
$$

respectively, where $C_{\leq 1}^{\infty}$ denotes the functions $T(\boldsymbol{x})$ which are polynomial of degree $\leq 1$ in $\boldsymbol{x}$. Comparing with table 1 , one can see that the $\mathfrak{s o}$ factors are the span of $\boldsymbol{J}$ and $\boldsymbol{P}$, whereas $C_{\leq 1}^{\infty}$ are spanned by $H$ and $\boldsymbol{B}$, which do indeed commute.

Let us now consider the carrollian conformal Killing vector fields. Let $\xi=\xi^{0} \frac{\partial}{\partial t}+\xi^{a} \frac{\partial}{\partial x^{a}}$ satisfy equation (10.4). The condition $\mathcal{L}_{\xi} \kappa=[\xi, \kappa]=-\frac{\lambda}{N} \kappa$ is satisfied provided that

$$
\frac{\partial \xi^{a}}{\partial t}=0 \quad \text { and } \quad \lambda=N\left(\frac{\partial \xi^{0}}{\partial t}+\frac{G(r)}{r} \boldsymbol{x} \cdot \boldsymbol{\xi}\right)
$$

where $\boldsymbol{x} \cdot \boldsymbol{\xi}:=x^{a} \xi^{a}$. The condition $\mathcal{L}_{\xi} b=\lambda b$ says that $\xi^{a} \frac{\partial}{\partial x^{a}}$ is a conformal Killing vector field of the metric $b$ with $\lambda=\frac{2}{D} \nabla_{a} \xi^{a}$, with $\nabla$ the Levi-Civita connection for $b$, which is the round metric on $\mathbb{S}^{D}$ for dSC, and the metric on hyperbolic space $\mathbb{H}^{D}$ for AdSC.

Let $D \geq 3$. Both $\mathbb{S}^{D}$ and $\mathbb{H}^{D}$ are conformally flat, so their Lie algebras of conformal Killing vector fields are isomorphic, and indeed isomorphic to that of $\mathbb{E}^{D}$ : namely, $\mathfrak{s o}(D+1,1)$.

Solving for $\xi^{0}$ we find

$$
\xi^{0}=T(\boldsymbol{x})+t\left(\frac{2}{N D} \operatorname{div} \xi-\frac{G(r)}{r} \boldsymbol{x} \cdot \boldsymbol{\xi}\right),
$$

where $\operatorname{div} \xi:=\nabla_{a} \xi^{a}$ and where $T$ is a smooth function on $\mathbb{S}^{D}$ or $\mathfrak{H}^{D}$ depending on whether we are in dSC or AdSC, respectively. As vector spaces, the Lie algebras $\mathfrak{c}^{\mathrm{dSC}}$ (resp. $\mathfrak{c}^{\text {AdSC }}$ ) of conformal symmetries of dSC (resp. AdSC) are isomorphic to $C^{\infty}\left(\mathbb{S}^{D}\right) \oplus \mathfrak{s o}(D+1,1)$ (resp. $C^{\infty}\left(\mathbb{H}^{D}\right) \oplus \mathfrak{s o}(D+1,1)$ ), with the isomorphism given by

$$
X \mapsto \xi_{X}+\left(\frac{2}{N D} \operatorname{div} \xi_{X}+\frac{G(r)}{r} \boldsymbol{x} \cdot \boldsymbol{\xi}_{X}\right) t \frac{\partial}{\partial t} \quad \text { and } \quad T \mapsto T \frac{\partial}{\partial t},
$$

for $X \in \mathfrak{s o}(D+1,1)$ and $T$ a smooth function in the relevant space.

As Lie algebras, $\mathfrak{c}^{\mathrm{dSC}}$ and $\mathfrak{c}^{\mathrm{AdSC}}$ are again semidirect products. Indeed, if $X \in \mathfrak{s o}(D+$ $1,1)$ and $f \in C^{\infty}$, then we find

$$
[X, T]=\xi_{X}(T)+\frac{G(r)}{r} \boldsymbol{x} \cdot \boldsymbol{\xi}_{X} T-\frac{2}{N D} \operatorname{div} \xi_{X} T .
$$

\footnotetext{
${ }^{11}$ Alternatively, we may view this "dressing" as a change of coordinates to a new rescaled time $t^{\prime}=-t g(r)$.
} 
If we again define $T \mapsto \widehat{T}=-C(r) T$, then

$$
\widehat{[X, T]}=\xi_{X}(\widehat{T})-\frac{2}{N D} \operatorname{div} \xi_{X} \widehat{T}
$$

so that $\widehat{T}$ is a section of the line bundle $\mathscr{L}^{\frac{2}{N}}$. In summary, just as in the case of the flat carrollian spacetime $\mathrm{C}$, we find that the Lie algebras $\mathfrak{c}^{\mathrm{dSC}}$ and $\mathfrak{c}^{\mathrm{AdSC}}$ are split extensions

$$
0 \longrightarrow \Gamma\left(\mathscr{L}^{\frac{2}{N}}\right) \longrightarrow \mathfrak{c}_{D \geq 3}^{(\mathrm{A}) \mathrm{dSC}} \longrightarrow \mathfrak{s o}(D+1,1) \longrightarrow 0
$$

where $\mathscr{L}$ is the density bundle on $\mathbb{S}^{D}$ or $\mathfrak{H}^{D}$ for dSC or AdSC, respectively. So again we see that in going from the Lie algebras of symmetries to the Lie algebras of conformal symmetries, all that happens is that the isometries enhance to conformal symmetries and what earlier were thought (after the "dressing") to be functions are actually sections of $\mathscr{L}^{\frac{2}{N}}$.

Now let $D=2$. Here the situation differs. As reviewed in appendix B, the case of dSC is just as for $D \geq 3$, whereas for AdSC, the Lie algebra of conformal Killing vector fields on $\mathbb{H}^{2}$ is enhanced to $\mathscr{O}(\mathbb{H})$, the holomorphic functions on the upper half-plane with the wronskian Lie bracket $[f, g]=f \partial g-g \partial f$. Therefore we have

$$
0 \longrightarrow \Gamma\left(\mathscr{L}^{\frac{2}{N}}\right) \longrightarrow \mathfrak{c}_{D=2}^{\mathrm{dSC}} \longrightarrow \mathfrak{s o}(3,1) \longrightarrow 0
$$

but

$$
0 \longrightarrow \Gamma\left(\mathscr{L}^{\frac{2}{N}}\right) \longrightarrow \mathfrak{c}_{D=2}^{\mathrm{AdSC}} \longrightarrow \mathscr{O}(\mathrm{H}) \longrightarrow 0
$$

For $D=1$ again every vector field is conformal Killing and their Lie algebra is isomorphic to the Lie algebra of smooth functions on the real line or the circle with the wronskian Lie bracket:

$$
0 \longrightarrow \Gamma\left(\mathscr{L}^{\frac{2}{N}}\right) \longrightarrow \mathfrak{c}_{D=1}^{\mathrm{d} S C} \longrightarrow C^{\infty}\left(S^{1}\right) \longrightarrow 0
$$

but

$$
0 \longrightarrow \Gamma\left(\mathscr{L}^{\frac{2}{N}}\right) \longrightarrow \mathfrak{c}_{D=1}^{\mathrm{AdSC}} \longrightarrow C^{\infty}(\mathbb{R}) \longrightarrow 0 .
$$

Let us restrict the discussion to $N=2$. Then the conformal symmetries of the dS carrollian structure are (at least in $3+1$ dimension) isomorphic to the BMS symmetries [35, 36] (for a definition of the BMS algebra in higher dimension see, e.g., [38]). This could have been anticipated since the dS carrollian structure is, up to a rescaling of time, the same as in [3]. It should however not be forgotten that dSC is a null surface in de Sitter spacetime and has nowhere vanishing curvature. For $D=2$, if we allow for conformal Killing vector fields on the sphere which are not everywhere smooth, then we may extend $\mathfrak{s l}(2, \mathbb{C})$ to "superrotations" [39, 40] (see also [41]). For $D=1$, the superrotations are built in from the start, which again is in agreement with the BMS group for $2+1$ "bulk" dimensions [38, 42].

Let us also observe that we find for the AdS carrollian spacetime in $D=2$, a null surface of AdS in $3+1$ dimensions, an infinite dimensional enhancement with "superrotations", in addition to the supertranslations. 


\subsection{Symmetries of the carrollian light cone (LC)}

These were already determined in [3], but we present it here for completeness. To determine the symmetries of the carrollian structure of LC, it is convenient to change coordinates.

Let $D \geq 2$. As shown in $[3,6]$, LC can be embedded as the future light cone in $(D+2)$ dimensional Minkowski spacetime $M^{D+2}$ in such a way that the carrollian structure is the one induced by the Minkowski metric on that null hypersurface. We may parametrise the future light cone in $\mathbb{M}^{D+2}$ by $\boldsymbol{x} \in \mathbb{R}^{D+1} \backslash\{0\}$ and the map $i: \mathbb{R}^{D+1} \backslash\{0\} \rightarrow \mathbb{M}^{D+2}$ is given by $i(\boldsymbol{x})=(r, \boldsymbol{x})$, where $r=\|\boldsymbol{x}\|>0$. The carrollian structure $(\kappa, b)$ is given by $\kappa=r \frac{\partial}{\partial r}$ and $b=i^{*} g$, where $g$ is the Minkowski metric:

$$
g=\eta_{\mu \nu} d X^{\mu} d X^{\nu}=-\left(d X^{0}\right)^{2}+\sum_{i}\left(d X^{i}\right)^{2}
$$

where the $X^{\mu}$ are the affine coordinates on $\mathbb{M}^{D+2}$. On the future light cone, $X^{0}=r$ and $X^{i}=x^{i}$. Therefore, we see that

$$
b=i^{*} g=-d r^{2}+\left(d r^{2}+r^{2} g_{\mathbb{S} D}\right)=r^{2} g_{\mathbb{S} D} .
$$

In terms of the coordinates $\boldsymbol{x}$, we have that $\kappa=x^{a} \frac{\partial}{\partial x^{a}}$ and

$$
b=\left(\delta_{a b}-\frac{x^{a} x^{b}}{r^{2}}\right) d x^{a} d x^{b}
$$

Now let $\xi=\xi^{a} \frac{\partial}{\partial x^{a}}$ be a symmetry of the carrollian structure $(\kappa, b)$. Then $\mathcal{L}_{\xi} \kappa=$ $[\xi, \kappa]=0$ and $\mathcal{L}_{\xi} b=0$. We find it more convenient to write

$$
\xi=\xi^{r} \frac{\partial}{\partial r}+\zeta
$$

where $\xi^{r} \in C^{\infty}\left(\mathbb{R}^{D+1} \backslash\{0\}\right)$ and $\zeta$ is a possibly $r$-dependent vector field tangent to the spheres of constant $r$; that is, $\zeta r=0$. The condition $[\kappa, \xi]=0$ results in

$$
0 \stackrel{!}{=}[\kappa, \xi]=\left[r \frac{\partial}{\partial r}, \xi^{r} \frac{\partial}{\partial r}+\zeta\right]=\left(r \frac{\partial \xi^{r}}{\partial r}-\xi^{r}\right) \frac{\partial}{\partial r}+r \frac{\partial \zeta}{\partial r} .
$$

This implies that $\xi^{r}=r F$, where $F \in C^{\infty}\left(\mathbb{S}^{D}\right)$, so that $\frac{\partial F}{\partial r}=0$, and $\zeta$ is independent of $r$. The condition $\mathcal{L}_{\xi} b=0$ results in

$$
0 \stackrel{!}{=} \mathcal{L}_{\xi}\left(r^{2} g_{S^{D}}\right)=2 r^{2} F g_{S D}+r^{2} \mathcal{L}_{\zeta} g_{\overparen{ } D}
$$

so that $\zeta$ is a conformal Killing vector on $\mathbb{S}^{D}$ and $F=-\frac{1}{D} \operatorname{div} \zeta$, where $\operatorname{div} \zeta$ is the intrinsic divergence of $\zeta$ on the sphere relative to the round metric, but which agrees with $\frac{\partial \zeta^{a}}{\partial x^{a}}$ in this case. Therefore, the symmetry algebra of the carrollian structure on LC is isomorphic to $\mathfrak{s o}(D+1,1)$, even for $D=2$ as shown in appendix $\mathrm{B}$, which is the transitive kinematical Lie algebra. It is an intriguing result that among the homogeneous carrollian spacetimes, it is precisely the non-reductive one whose symmetry algebra is finite-dimensional. 
For $D=1$, LC is the universal cover of the future light cone in three-dimensional Minkowski spacetime. One can model LC as the submanifold of $\mathbb{R}^{3}$ with points

$$
\mathbf{L C}=\{(r \cos \theta, r \sin \theta, \theta) \mid r>0, \theta \in \mathbb{R}\},
$$

with the covering map from LC to the future light cone in $\mathbb{M}^{3}$ given by $(r \cos \theta, r \sin \theta, \theta) \mapsto$ $(r, r \cos \theta, r \sin \theta)$. Notice that the non-contractible circles of constant $r$ in the light cone lift to contractible helices in LC. The transitive kinematical Lie algebra is isomorphic to $\mathfrak{s l}(2, \mathbb{R})$ and is spanned by the vector fields

$$
\frac{\partial}{\partial \theta}, \quad \cos \theta \frac{\partial}{\partial \theta}+r \sin \theta \frac{\partial}{\partial r} \quad \text { and } \quad \sin \theta \frac{\partial}{\partial \theta}-r \cos \theta \frac{\partial}{\partial r} .
$$

Since they are periodic in $\theta$ with period $2 \pi$, they descend to tangent vector fields to the future light cone. The carrollian structure is given by $\kappa=r \frac{\partial}{\partial r}$ and $b=r^{2} d \theta^{2}$, except that $\theta$ is not angular in LC. It is straightforward to work out the Lie algebra of carrollian Killing vector fields and obtain that it is isomorphic to $C^{\infty}\left(\mathbb{R}_{\theta}\right)$ with the wronskian Lie bracket. Indeed, if $f \in C^{\infty}\left(\mathbb{R}_{\theta}\right)$, the corresponding vector field is

$$
\xi_{f}=f(\theta) \frac{\partial}{\partial \theta}-f^{\prime}(\theta) r \frac{\partial}{\partial r}
$$

and the Lie bracket is given by

$$
\left[\xi_{f}, \xi_{g}\right]=\xi_{h} \quad \text { with } \quad h=f g^{\prime}-f^{\prime} g .
$$

For the (non-simply connected) future light cone, we must consider periodic functions, so that the Lie algebra of carrollian Killing vector fields is $C^{\infty}\left(S^{1}\right)$ with the wronskian Lie bracket.

Let us now consider the carrollian conformal Killing vector fields. Again we first consider $D \geq 2$. This was treated already in [3], but we write it here for completeness. As before we work in embedding coordinates where the carrollian structure on LC is given by

$$
\kappa=r \frac{\partial}{\partial r} \quad \text { and } \quad b=r^{2} g_{\mathbb{S} D}
$$

and let $\xi=\xi^{r} \frac{\partial}{\partial r}+\zeta$, with $\zeta r=0$, satisfy equation (10.4). The condition $\mathcal{L}_{\xi} \kappa=-\frac{\lambda}{N} \kappa$ results in

$$
\frac{\partial \zeta}{\partial r}=0 \quad \text { and } \quad r \frac{\partial \xi^{r}}{\partial r}-\xi^{r}=\frac{\lambda}{N} r
$$

whereas the condition $\mathcal{L}_{\xi} b=\lambda b$ results in

$$
\mathcal{L}_{\zeta} g_{\mathbb{S} D}=\left(\lambda-\frac{2 \xi^{r}}{r}\right) g_{\mathbb{S} D}
$$

so that $\zeta$ is a conformal Killing vector field on $\mathbb{S}^{D}$ with divergence

$$
\operatorname{div} \zeta=\frac{D}{2}\left(\lambda-\frac{2 \xi^{r}}{r}\right) .
$$


Solving for $\xi^{r}$ we find

$$
\xi^{r}=r\left(r^{\frac{N}{2}} T-\frac{1}{D} \operatorname{div} \zeta\right)
$$

for some $T \in C^{\infty}\left(\mathbb{S}^{D}\right)$. Therefore, as a vector space, the Lie algebra $\mathfrak{c}^{\mathrm{LC}}$ of carrollian conformal Killing vector fields of LC is isomorphic to $C^{\infty}\left(\mathbb{S}^{D}\right) \oplus \mathfrak{s o}(D+1,1)$, with the isomorphism given by

$$
X \mapsto \zeta_{X}-\frac{1}{D} \operatorname{div} \zeta_{X} r \frac{\partial}{\partial r} \quad \text { and } \quad T \mapsto r^{\frac{2}{N}} \operatorname{Tr} \frac{\partial}{\partial r},
$$

for $X \in \mathfrak{s o}(D+1,1)$ and $T \in C^{\infty}\left(\mathbb{S}^{D}\right)$.

As a Lie algebra, $\mathfrak{c}^{\mathrm{LC}}$ is a semi-direct product with

$$
[X, T]=\zeta_{X}(T)-\frac{2}{N D} \operatorname{div} \zeta_{X} T
$$

so that $T$ is actually a section of $\mathscr{L}^{\frac{2}{N}}$. In summary, $\mathfrak{c}^{\mathrm{LC}}$ is a split extension

$$
0 \longrightarrow \Gamma\left(\mathscr{L}^{\frac{2}{N}}\right) \longrightarrow \mathfrak{c}^{\mathrm{LC}} \longrightarrow \mathfrak{s o}(D+1,1) \longrightarrow 0
$$

which shows that there is an isomorphism $\mathfrak{c}^{\mathrm{LC}} \cong \mathfrak{c}^{\mathrm{dSC}}$.

For $D=1$, analogous to the case of carrollian Killing vector fields, we find that now the Lie algebra of carrollian conformal Killing vector fields is larger. The carrollian conformal Killing vector fields at level $N$ are given by

$$
f(\theta) \frac{\partial}{\partial \theta}-f^{\prime}(\theta) r \frac{\partial}{\partial r}+r^{\frac{2}{N}} g(\theta) r \frac{\partial}{\partial r}
$$

for some $f, g \in C^{\infty}\left(\mathbb{R}_{\theta}\right)$. The Lie algebra structure is now a semidirect product of the wronskian Lie algebra $C^{\infty}\left(\mathbb{R}_{\theta}\right)$ of carrollian Killing vector fields and the abelian ideal of sections of $\mathscr{L}^{\frac{2}{N}}$ :

$$
0 \longrightarrow \Gamma\left(\mathscr{L}^{\frac{2}{N}}\right) \longrightarrow \mathfrak{c}_{D=1}^{\mathrm{LC}} \longrightarrow C^{\infty}\left(\mathbb{R}_{\theta}\right) \longrightarrow 0
$$

where under the isomorphism $\mathscr{L}^{\frac{2}{N}} \cong C^{\infty}\left(\mathbb{R}_{\theta}\right)$, to a function $g \in C^{\infty}\left(\mathbb{R}_{\theta}\right)$ there corresponds the vector field $\zeta_{g}=r^{\frac{2}{N}} g(\theta) r \frac{\partial}{\partial r}$, so that with $\xi_{f}=f(\theta) \frac{\partial}{\partial \theta}-f^{\prime}(\theta) r \frac{\partial}{\partial r}$, we have

$$
\left[\xi_{f}, \zeta_{g}\right]=\zeta_{h} \quad \text { with } \quad h=f g^{\prime}-\frac{2}{N} f^{\prime} g
$$

\subsection{Symmetries of galilean structures}

In this section, we will work out the Lie algebra of galilean Killing vector fields for the homogeneous galilean spacetimes. This Lie algebra has been termed the Coriolis algebra of a galilean spacetime in [37]. In the modified exponential coordinates of appendix A, the invariant galilean structure takes the same form in all the homogeneous spacetimes G, dSG, AdSG, dSG $\gamma, \mathrm{AdSG}_{\chi}$ and $\mathrm{S} 12_{\gamma, \chi}$ : the clock one-form is given by $\tau=d t$ and the inverse spatial metric by $h=\delta^{a b} \frac{\partial}{\partial x^{a}} \otimes \frac{\partial}{\partial x^{b}}$. 
Let $\xi=\xi^{0} \frac{\partial}{\partial t}+\xi^{a} \frac{\partial}{\partial x^{a}}$ satisfy equation (10.1). The condition that $\xi$ preserves the clock-one form says

$$
0 \stackrel{!}{=} \mathcal{L}_{\xi} \tau=\mathcal{L}_{\xi} d t=d \mathcal{L}_{\xi} t=d \xi^{0} \Longrightarrow \xi^{0} \text { is constant. }
$$

The condition that $\mathcal{L}_{\xi} h=0$ says that

$$
0 \stackrel{!}{=} \mathcal{L}_{\xi} h=-\left(\partial_{a} \xi_{b}+\partial_{b} \xi_{a}\right) \frac{\partial}{\partial x^{a}} \otimes \frac{\partial}{\partial x^{b}} \Longrightarrow \partial_{a} \xi_{b}+\partial_{b} \xi_{a}=0
$$

This equation says that $\xi^{a} \partial_{a}$ is a (possibly) $t$-dependent Killing vector field of the $D$ dimensional euclidean space $\mathbb{E}^{D}$, so that

$$
\xi^{a}(x, t)=f^{a}(t)+\Lambda_{b}^{a}(t) x^{b},
$$

where $\Lambda_{a b}=-\Lambda_{b a}$. In other words,

$$
\xi=\xi^{0} \frac{\partial}{\partial t}+f^{a}(t) \frac{\partial}{\partial x^{a}}+\Lambda(t)^{a}{ }_{b} x^{b} \frac{\partial}{\partial x^{a}},
$$

so that, as a vector space, the Lie algebra $\mathfrak{a}$ of vector fields which preserve the galilean structure $(\tau, h)$, is isomorphic to $\mathfrak{a} \cong \mathbb{R} \oplus C^{\infty}\left(\mathbb{R}_{t}, \mathfrak{e}\right)$, with $\mathfrak{e}$ the euclidean Lie algebra and $\mathbb{R}_{t}$ the real line with coordinate $t$. As a Lie algebra,

$$
\mathfrak{a} \cong \mathbb{R} \ltimes C^{\infty}\left(\mathbb{R}_{t}, \mathfrak{e}\right)
$$

has the structure of a semidirect product or, equivalently, a split extension

$$
0 \longrightarrow C^{\infty}\left(\mathbb{R}_{t}, \mathfrak{e}\right) \longrightarrow \mathfrak{a} \longrightarrow \mathbb{R} \longrightarrow 0
$$

where the splitting $\mathbb{R} \rightarrow \mathfrak{a}$ is given by sending $1 \in \mathbb{R}$ to $\frac{\partial}{\partial t}$, corresponding to the action of $H$. This was originally worked out in [37], who named it the Coriolis algebra.

We will now determine the Lie algebra $\mathfrak{c}$ of conformal symmetries of the galilean structure and we will see that it has a very similar structure to $\mathfrak{a}$ in equation (10.68), except that $\mathbb{R}$ gets enhanced to a non-abelian Lie algebra structure on $C^{\infty}\left(\mathbb{R}_{t}\right)$.

Let $\xi=\xi^{0} \frac{\partial}{\partial t}+\xi^{a} \frac{\partial}{\partial x^{a}}$ satisfy equation (10.2). The condition $\mathcal{L}_{\xi} \tau=-\frac{\lambda}{N} \tau$ results in

$$
\frac{\partial \xi^{0}}{\partial x^{a}}=0 \quad \text { and } \quad \frac{\partial \xi^{0}}{\partial t}=-\frac{\lambda}{N} \Longrightarrow \lambda=\lambda(t) .
$$

The condition $\mathcal{L}_{\xi} h=\lambda h$ results in

$$
\frac{\partial \xi^{a}}{\partial x^{b}}+\frac{\partial \xi^{b}}{\partial x^{a}}=-\lambda \delta_{a b}
$$

so that $\xi^{a} \frac{\partial}{\partial x^{a}}$ is a (possibly) $t$-dependent conformal Killing vector field on $\mathbb{E}^{D}$, but since $\lambda=\lambda(t)$, we see that that $\xi^{a} \frac{\partial}{\partial x^{a}}$ is either Killing or homothetic. In other words, we can write

$$
\xi^{a}=f^{a}(t)+\Lambda_{b}^{a}(t) x^{b}+g^{\prime}(t) x^{a}
$$


where we have found it convenient to think of the homothetic component as the derivative of a smooth function $g \in C^{\infty}\left(\mathbb{R}_{t}\right)$. Doing so, we may solve for $\xi^{0}$ to arrive at

$$
\xi^{0}=-\frac{2}{N D} g(t)
$$

so that

$$
\xi=\left(f^{a}(t)+\Lambda_{b}^{a}(t) x^{b}\right) \frac{\partial}{\partial x^{a}}+\left(-\frac{2}{N D} g(t) \frac{\partial}{\partial t}+g^{\prime}(t) x^{a} \frac{\partial}{\partial x^{a}}\right),
$$

in agreement with [43, eq. (3.12)] and [29, eq. (III.5)], who worked out the case of G.

Thus we see that, as a vector space, the Lie algebra $\mathfrak{c}$ of conformal symmetries of the galilean spacetime is isomorphic to $C^{\infty}\left(\mathbb{R}_{t}, \mathfrak{e}\right) \oplus C^{\infty}\left(\mathbb{R}_{t}\right)$, with the isomorphism such that $g \in C^{\infty}\left(\mathbb{R}_{t}\right)$ is sent to the vector field

$$
g(t) \mapsto g^{\prime}(t) x^{a} \frac{\partial}{\partial x^{a}}-\frac{2}{N D} g(t) \frac{\partial}{\partial t} .
$$

In particular, the Lie algebra structure on $C^{\infty}\left(\mathbb{R}_{t}\right)$ is not abelian, but rather if $f, g \in$ $C^{\infty}\left(\mathbb{R}_{t}\right)$, their Lie bracket is a multiple of the wronskian:

$$
[f, g]=\frac{-2}{N D}\left(f g^{\prime}-f^{\prime} g\right)
$$

As a Lie algebra, $\mathfrak{c}$ is a semidirect product, where $f \in C^{\infty}\left(\mathbb{R}_{t}\right)$ acts on $(\boldsymbol{v}(t), \Lambda(t)) \in$ $C^{\infty}\left(\mathbb{R}_{t}, \mathfrak{e}\right)$ by

$$
[f,(\boldsymbol{v}, \Lambda)]=\left(\frac{-2}{N D} f \boldsymbol{v}^{\prime}+f^{\prime} \boldsymbol{v}, \frac{-2}{N D} f \Lambda^{\prime}\right) .
$$

In summary, the Lie algebra $\mathfrak{c}$ is a split extension

$$
0 \longrightarrow C^{\infty}\left(\mathbb{R}_{t}, \mathfrak{e}\right) \longrightarrow \mathfrak{c} \longrightarrow C^{\infty}\left(\mathbb{R}_{t}\right) \longrightarrow 0,
$$

so that in going from the symmetries to the conformal symmetries, the abelian Lie algebra $\mathbb{R}$ has been enhanced to the non-abelian "wronskian" Lie algebra $C^{\infty}\left(\mathbb{R}_{t}\right)$.

It is intriguing that the galilean spacetimes, despite admitting non-isomorphic transitive kinematical Lie algebras, have isomorphic conformal symmetry Lie algebras. It would be interesting to investigate how the transitive Lie algebras relate via their embeddings in $\mathfrak{c}$.

\section{Conclusions}

The main results of this and our previous paper [6] are

1. the classification of simply-connected spatially isotropic homogeneous spacetimes, recorded in tables 1 and 2 ;

2. the proof that the boosts act with generic non-compact orbits on all spacetimes in table 1 except for the riemannian symmetric spaces, and

3. the determination of the Lie algebra of infinitesimal (conformal) symmetries of these structures. 
The second point is an important physical requirement, already mentioned in [1]. We also discussed the subtle interplay between the kinematical Lie algebras and their spacetimes [6]. Among them is the intriguing connection between the anti de Sitter carrollian and Minkowski spacetime, which are different homogeneous spacetimes, but based on the same Lie algebra.

In addition, we also determined the invariant affine connections on these homogeneous spacetimes and calculated their torsion and curvature. These connections allow us to define geodesics, which we hope to study in future work.

Table 3 summarises the basic geometric properties of the spacetimes. This table makes it clear that the bulk of the spacetimes do not admit an invariant metric and hence that there is a very rich landscape beyond lorentzian geometry, even if we remain within the realm of homogeneous spaces with space isotropy.

Another aspect of this work was the analysis of the, generically infinite dimensional, (conformal) symmetries of the carrollian and galilean structures. One observation is that the Lie algebra of infinitesimal conformal symmetries of carrollian (anti) de Sitter spacetime, which embeds as a null hypersurface of (anti) de Sitter spacetime, is infinite dimensional and reminiscent of the BMS algebra. It is tempting to speculate that this might be relevant for BMS physics (memory effect, ...) [4, 5] on these non-flat backgrounds (see also [7]).

Some of the above results were made possible by the introduction of local coordinates. We chose to consider exponential coordinates; although admittedly these are not always the simplest coordinates for calculations. We have found modified exponential coordinates to be quite useful as well, particularly for the determination of the infinitesimal (conformal) symmetries of the spacetimes. We expressed the kinematical vector fields - that is, the infinitesimal generators of rotations, boosts and translations - in terms of exponential coordinates, and we did the same for the invariant structures (if any). This was particularly useful in order to determine their infinitesimal (conformal) symmetries.

There are a number of possible directions for future research departing from our results.

One open problem we did not address is to exhibit the galilean spacetimes as null reductions of lorentzian spacetimes in one higher dimension. This would complement the description of the carrollian spacetimes as null hypersurfaces in an ambient lorentzian manifold.

We showed that all of the galilean spacetimes in this paper ( G, dSG, AdSG, dSG $\gamma$, AdSG $\chi$ and $\mathrm{S} 12_{\gamma, \chi}$ ) have isomorphic Lie algebras of infinitesimal conformal symmetries. We did not determine how the transitive kinematical Lie algebras are embedded in these infinitedimensional Lie algebras. Perhaps studying those embeddings might teach us something about how the kinematical Lie algebras relate to each other.

It would be interesting to promote the homogeneous spacetimes to Cartan geometries and hence study the possible theories based on them. For a discussion in $2+1$ dimensions see [44].

Another intriguing direction is to explore the applications of these geometries to nonAdS holography. It is not inconceivable that some of these homogeneous geometries might play a similar rôle in non-AdS holography to that played by anti de Sitter spacetime in 
the AdS/CFT correspondence [2]. One particularly interesting property of a non-zero cosmological constant is that acts as an infrared regulator (often paraphrased as "AdS is like a box") and it would be interesting to investigate if this persists in the non-relativistic or ultra-relativistic limits to AdSG or AdSC, respectively.

\section{Acknowledgments}

During the embryonic stages of this work, JMF and SP were participating at the MITP Topical Workshop "Applied Newton-Cartan Geometry" (APPNC2018), held at the Mainz Institute for Theoretical Physics, to whom we are grateful for their support, their hospitality and for providing such a stimulating research atmosphere. We are particularly grateful to Eric Bergshoeff and Niels Obers for the invitation to participate. We are grateful to Yvonne Calò for checking some calculations in the paper. JMF would like to acknowledge helpful conversations with Jelle Hartong, James Lucietti and Michael Singer. SP is grateful to Glenn Barnich, Carlo Heissenberg, Marc Henneaux, Yegor Korovin, Javier Matulich, Arash Ranjbar, Jan Rosseel, Romain Ruzziconi and Jakob Salzer for useful discussions.

The research of JMF is partially supported by the grant ST/L000458/1 "Particle Theory at the Higgs Centre" from the U.K. Science and Technology Facilities Council. The research of SP is partially supported by the ERC Advanced Grant "High-Spin-Grav" and by FNRS-Belgium (convention FRFC PDR T.1025.14 and convention IISN 4.4503.15). SP acknowledges support from the Erwin Schrödinger Institute during his stay at the "Higher Spins and Holography" workshop.

SP wants to dedicate this work to his "kleine Oma" Amelie Prohazka.

\section{A Modified exponential coordinates}

In this appendix we revisit the local geometry of the homogeneous carrollian and galilean spacetimes, but this time in modified exponential coordinates.

\section{A.1 Carrollian spacetimes}

\section{A.1.1 Carrollian (anti) de Sitter spacetimes}

Let $\sigma^{\prime}(t, \boldsymbol{x})=\exp (t H) \exp (\boldsymbol{x} \cdot \boldsymbol{P}) \cdot o$. We calculate the soldering form by pulling back the left-invariant Maurer-Cartan one-form $\vartheta$ on the Lie group:

$$
\sigma^{\prime *}(\vartheta)=\theta+\omega=\exp \left(-\operatorname{ad}_{A}\right) H d t+D\left(\operatorname{ad}_{A}\right)(d \boldsymbol{x} \cdot \boldsymbol{P}),
$$

where $A=\boldsymbol{x} \cdot \boldsymbol{P}$. We find

$$
\operatorname{ad}_{A} H=\varepsilon \boldsymbol{x} \cdot \boldsymbol{B} \quad \text { and } \quad \operatorname{ad}_{A}^{2} H=-\varepsilon x^{2} H,
$$

so that

$$
\exp \left(-\operatorname{ad}_{A}\right) H=\cosh \left(x_{+}\right) H-\frac{\sinh \left(x_{+}\right)}{x_{+}} \varepsilon \boldsymbol{x} \cdot \boldsymbol{B},
$$


where $x_{+}^{2}=-\varepsilon x^{2}$ and $x_{-}=-x_{+}$. Also, we find

$$
\begin{aligned}
\operatorname{ad}_{A} P_{a} & =\varepsilon J_{a b} x^{b} \\
\operatorname{ad}_{A}^{2} P_{a} & =-\varepsilon x^{2} P_{a}+\varepsilon x^{a} A \\
\Longrightarrow \operatorname{ad}_{A}^{3} P_{a} & =-\varepsilon x^{2} \operatorname{ad}_{A} P_{a} .
\end{aligned}
$$

Therefore,

$$
D\left(\operatorname{ad}_{A}\right) P_{a}=P_{a}+D^{-} \varepsilon x^{b} J_{a b}+\frac{1}{x_{+}^{2}}\left(D^{+}-1\right)\left(-\varepsilon x^{2} P_{a}+\varepsilon x^{a} \boldsymbol{x} \cdot \boldsymbol{P}\right),
$$

where $D^{-}=\frac{1}{2 x_{+}}\left(D\left(x_{+}\right)-D\left(x_{-}\right)\right)$and $D^{+}=\frac{1}{2}\left(D\left(x_{+}\right)+D\left(x_{-}\right)\right)$. In summary,

$$
\theta=\cosh \left(x_{+}\right) d t H+D^{+} d \boldsymbol{x} \cdot \boldsymbol{P}+\frac{\varepsilon}{x_{+}^{2}}\left(D^{+}-1\right) \boldsymbol{x} \cdot d \boldsymbol{x} \boldsymbol{x} \cdot \boldsymbol{P} .
$$

Using that $D^{+}=\frac{\sinh \left(x_{+}\right)}{x_{+}}$, we find

$$
\theta=\cosh \left(x_{+}\right) d t H+\frac{\sinh \left(x_{+}\right)}{x_{+}} d \boldsymbol{x} \cdot \boldsymbol{P}+\varepsilon \frac{\sinh \left(x_{+}\right)-x_{+}}{x_{+}^{3}} \boldsymbol{x} \cdot d \boldsymbol{x} \boldsymbol{x} \cdot \boldsymbol{P} .
$$

The carrollian structure is given by $\kappa=E_{H}=\operatorname{sech}\left(x_{+}\right) \frac{\partial}{\partial t}$ and $b=\pi^{2}(\theta, \theta)$, which expands to

$$
b=-\frac{\varepsilon}{x^{2}} \sinh ^{2}\left(x_{+}\right) d \boldsymbol{x} \cdot d \boldsymbol{x}+\frac{\varepsilon}{x^{4}}\left(\sinh ^{2}\left(x_{+}\right)-x_{+}^{2}\right)(\boldsymbol{x} \cdot d \boldsymbol{x})^{2} .
$$

If $\varepsilon=1, x_{+}=i r$, where $r=|\boldsymbol{x}|$ and hence $\sinh ^{2} x_{+}=-\sin ^{2} r$, so that

$$
b=\frac{\sin ^{2} r}{r^{2}}\left(d r^{2}+r^{2} g_{S^{D-1}}\right)-\frac{\left(\sin ^{2} r-r^{2}\right)}{r^{2}} d r^{2}=d r^{2}+\sin ^{2} r g_{S^{D-1}},
$$

which is the round metric on $\mathbb{S}^{D}$. The coordinate system is good provided that $r \in\left(0, \frac{\pi}{2}\right)$. On the other hand, if $\varepsilon=-1, x_{+}=r$ and, therefore,

$$
b=\frac{\sinh ^{2} r}{r^{2}}\left(d r^{2}+r^{2} g_{\mathbb{S}^{D-1}}\right)-\frac{\left(\sinh ^{2} r-r^{2}\right)}{r^{2}} d r^{2}=d r^{2}+\sinh ^{2} r g_{\mathbb{S}^{D-1}},
$$

which is the metric on hyperbolic space $\uplus^{D}$ and the coordinate system is good for all $r>0$. In summary, the carrollian structures in these coordinate systems are given by

$$
\begin{aligned}
& \kappa^{\mathrm{dSC}}=\sec (r) \frac{\partial}{\partial t} \\
& b^{\mathrm{dSC}}=d r^{2}+\sin ^{2} r g_{\mathbb{S} D-1} \\
& \kappa^{\mathrm{AdSC}}=\operatorname{sech}(r) \frac{\partial}{\partial t} \\
& b^{\mathrm{AdSC}}=d r^{2}+\sinh ^{2} r g_{\mathbb{S}^{D-1}} .
\end{aligned}
$$

\section{A.2 Galilean spacetimes}

The transitive kinematical Lie algebras for the homogeneous galilean spacetimes (with the exception of $\mathrm{S} 12_{\gamma, \chi}$, which will be treated separately below) has additional brackets of the form

$$
[H, \boldsymbol{B}]=-\boldsymbol{P} \quad \text { and } \quad[H, \boldsymbol{P}]=\alpha \boldsymbol{B}+\beta \boldsymbol{P},
$$


for some $\alpha, \beta \in \mathbb{R}$. In other words, $\operatorname{ad}_{H}$ is represented by a matrix of the form $\left(\begin{array}{cc}0 & \alpha \\ -1 & \beta\end{array}\right)$. We define

$$
M(t)=\exp \left(t\left(\begin{array}{cc}
0 & \alpha \\
-1 & \beta
\end{array}\right)\right) .
$$

We introduce modified exponential coordinates $(t, \boldsymbol{x})$ by acting with $L(t, \boldsymbol{x}):=$ $\exp (t H) \exp (\boldsymbol{x} \cdot \boldsymbol{P})$ on the origin $o$. Relative to them $\xi_{H}=\frac{\partial}{\partial t}$ and $\xi_{J_{a b}}$ are as in exponential coordinates. We will determine $\xi_{B_{a}}$ and then calculate $\xi_{P_{a}}=\left[\xi_{H}, \xi_{B_{a}}\right]$.

Let $s \in(-\varepsilon, \varepsilon)$ and consider

$$
\exp (s \boldsymbol{v} \cdot \boldsymbol{B}) L(t, \boldsymbol{x}) \cdot o=L(\tau(s), \boldsymbol{y}(s)) \cdot o,
$$

where $\tau(0)=t$ and $\boldsymbol{y}(0)=\boldsymbol{x}$. This is equivalent to

$$
\exp (s \boldsymbol{v} \cdot \boldsymbol{B}) L(t, \boldsymbol{x})=L(\tau(s), \boldsymbol{y}(s)) \exp (\boldsymbol{w}(s) \cdot \boldsymbol{B}),
$$

where $\boldsymbol{w}(0)=0$, which we may re-write yet again as

$$
\exp (\tau(s) H) \exp (\boldsymbol{y}(s) \cdot \boldsymbol{P})=\exp (s \boldsymbol{v} \cdot \boldsymbol{B}) L(t, \boldsymbol{x}) \exp (-\boldsymbol{w}(s) \cdot \boldsymbol{B}) .
$$

We now differentiate with respect to $s$ at $s=0$ to obtain (in the notation of matrix groups)

$$
\left(\tau^{\prime}(0) H\right) L(t, \boldsymbol{x})+L(t, \boldsymbol{x})\left(\boldsymbol{y}^{\prime}(0) \cdot \boldsymbol{P}\right)=(\boldsymbol{v} \cdot \boldsymbol{B}) L(t, \boldsymbol{x})-L(t, \boldsymbol{x})\left(\boldsymbol{w}^{\prime}(0) \cdot \boldsymbol{B}\right),
$$

which implies that $\tau^{\prime}(0)=0$ and

$$
\boldsymbol{y}^{\prime}(0) \cdot \boldsymbol{P}+\boldsymbol{w}^{\prime}(0) \cdot \boldsymbol{B}=M(-t) \boldsymbol{v} \cdot \boldsymbol{B} \quad \text { or } \quad\left(\begin{array}{l}
\boldsymbol{w}^{\prime}(0) \\
\boldsymbol{y}^{\prime}(0)
\end{array}\right)=M(-t)\left(\begin{array}{l}
\boldsymbol{v} \\
\mathbf{0}
\end{array}\right) .
$$

We now proceed to treat the different galilean spacetimes in turn, but first we simply comment on the fact that the galilean structure is formally identical in all cases. Indeed,

$$
L(t, \boldsymbol{x})^{-1} d L(t, \boldsymbol{x})=H d t+\left(\beta d t x^{a}+d x^{a}\right) P_{a}+\alpha d t x^{a} B_{a},
$$

where $[H, \boldsymbol{P}]=\alpha \boldsymbol{B}+\beta \boldsymbol{P}$ defines $\alpha$ and $\beta$. It follows from this that the soldering form is given by

$$
\theta^{H}=d t \quad \text { and } \quad \theta^{P_{a}}=d x^{a}+\beta x^{a} d t
$$

the invariant canonical connection by

$$
\omega=\alpha d t \boldsymbol{x} \cdot \boldsymbol{B}
$$

and the vielbein is

$$
E_{H}=\frac{\partial}{\partial t}-\beta x^{a} \frac{\partial}{\partial x^{a}} \quad \text { and } \quad E_{P_{a}}=\frac{\partial}{\partial x^{a}} .
$$

The galilean structure is given by the clock one-form

$$
\eta(\theta)=d t
$$


and the inverse spatial metric

$$
\delta^{a b} E_{P_{a}} \otimes E_{P_{b}}=\delta^{a b} \frac{\partial}{\partial x^{a}} \otimes \frac{\partial}{\partial x^{b}} .
$$

The torsion and curvature are, respectively

$$
\Theta=-\beta d t \wedge d \boldsymbol{x} \cdot \boldsymbol{P} \quad \text { and } \quad \Omega=-\alpha d t \wedge d \boldsymbol{x} \cdot \boldsymbol{B} .
$$

We now work out the expressions of the fundamental vector fields $\xi_{B_{a}}$ and $\xi_{P_{a}}$ in each case.

\section{A.2.1 Galilean spacetime}

For the galilean spacetime G,

$$
M(t)=\exp \left(t\left(\begin{array}{cc}
0 & 0 \\
-1 & 0
\end{array}\right)\right)=\left(\begin{array}{cc}
1 & 0 \\
-t & 1
\end{array}\right),
$$

and hence

$$
\left(\begin{array}{c}
\boldsymbol{w}^{\prime}(0) \\
\boldsymbol{y}^{\prime}(0)
\end{array}\right)=\left(\begin{array}{c}
\boldsymbol{v} \\
t \boldsymbol{v}
\end{array}\right)
$$

from where we read off

$$
\xi_{B_{a}}=t \frac{\partial}{\partial x^{a}} \quad \text { and hence } \quad \xi_{P_{a}}=\frac{\partial}{\partial x^{a}} .
$$

\section{A.2.2 Galilean de Sitter spacetime}

For the galilean de Sitter spacetime dSG,

$$
M(t)=\left(\begin{array}{cc}
\cosh t & -\sinh t \\
-\sinh t & \cosh t
\end{array}\right),
$$

and hence

$$
\left(\begin{array}{l}
\boldsymbol{w}^{\prime}(0) \\
\boldsymbol{y}^{\prime}(0)
\end{array}\right)=\left(\begin{array}{l}
\boldsymbol{v} \cosh t \\
\boldsymbol{v} \sinh t
\end{array}\right)
$$

from where we read off

$$
\xi_{B_{a}}=\sinh t \frac{\partial}{\partial x^{a}} \quad \text { and hence } \quad \xi_{P_{a}}=\cosh t \frac{\partial}{\partial x^{a}} .
$$

\section{A.2.3 Torsional galilean de Sitter spacetime}

For the torsional galilean de Sitter spacetime $\mathrm{dSG}_{\gamma}$,

$$
M(t)=\frac{1}{1-\gamma}\left(\begin{array}{cc}
e^{t \gamma}-\gamma e^{t} & \gamma\left(e^{t}-e^{t \gamma}\right) \\
e^{t \gamma}-e^{t} & e^{t}-\gamma e^{t \gamma}
\end{array}\right),
$$

and hence

$$
\left(\begin{array}{l}
\boldsymbol{w}^{\prime}(0) \\
\boldsymbol{y}^{\prime}(0)
\end{array}\right)=\frac{1}{1-\gamma}\left(\begin{array}{c}
\left(e^{t \gamma}-\gamma e^{t}\right) \boldsymbol{v} \\
\left(e^{t \gamma}-e^{t}\right) \boldsymbol{v}
\end{array}\right)
$$

from where we read off

$$
\xi_{B_{a}}=\frac{e^{t \gamma}-e^{t}}{1-\gamma} \frac{\partial}{\partial x^{a}} \quad \text { and hence } \quad \xi_{P_{a}}=\frac{\gamma e^{t \gamma}-e^{t}}{1-\gamma} \frac{\partial}{\partial x^{a}} .
$$




\section{A.2.4 Galilean anti de Sitter spacetime}

For the galilean anti de Sitter spacetime AdSG,

$$
M(t)=\left(\begin{array}{cc}
\cos t & \sin t \\
-\sin t & \cos t
\end{array}\right),
$$

and hence

$$
\left(\begin{array}{l}
\boldsymbol{w}^{\prime}(0) \\
\boldsymbol{y}^{\prime}(0)
\end{array}\right)=\left(\begin{array}{l}
\boldsymbol{v} \cos t \\
\boldsymbol{v} \sin t
\end{array}\right)
$$

from where we read off

$$
\xi_{B_{a}}=\sin t \frac{\partial}{\partial x^{a}} \quad \text { and hence } \quad \xi_{P_{a}}=\cos t \frac{\partial}{\partial x^{a}} .
$$

\section{A.2.5 Torsional galilean anti de Sitter spacetime}

For the torsional galilean anti de Sitter spacetime $\mathrm{AdSG}_{\chi}$,

$$
M(t)=\left(\begin{array}{cc}
e^{t \chi}(\cos t-\chi \sin t) & e^{t \chi}\left(1+\chi^{2}\right) \sin t \\
-e^{t \chi}\left(1+\chi^{2}\right) \sin t & e^{t \chi}(\cos t+\chi \sin t)
\end{array}\right),
$$

and hence

$$
\left(\begin{array}{c}
\boldsymbol{w}^{\prime}(0) \\
\boldsymbol{y}^{\prime}(0)
\end{array}\right)=\left(\begin{array}{c}
e^{-t \chi}(\cos t+\chi \sin t) \boldsymbol{v} \\
e^{-t \chi} \sin t \boldsymbol{v}
\end{array}\right)
$$

from where we read off

$$
\xi_{B_{a}}=e^{-t \chi} \sin t \frac{\partial}{\partial x^{a}} \quad \text { and hence } \quad \xi_{P_{a}}=e^{-t \chi}(\cos t-\chi \sin t) \frac{\partial}{\partial x^{a}} .
$$

\section{A.2.6 Spacetime $\mathrm{S}_{2} 2_{\gamma, \chi}$}

For spacetime $\mathrm{S} 12_{\gamma, \chi}$, the expression for the fundamental vector fields $\xi_{B_{a}}$ and $\xi_{P_{a}}$ are not particularly transparent in modified exponential coordinates, so we will not give them here. We will show, however, that the galilean structure is formally identical to that of all the other homogeneous galilean spacetimes.

The transitive Lie algebra in this case is defined by the following brackets

$$
\left[H, B_{a}\right]=-P_{a} \quad \text { and } \quad\left[H, P_{a}\right]=(1+\gamma) P_{a}+\gamma B_{a}-\chi \epsilon_{a b}\left(B_{b}+P_{b}\right) .
$$

Letting $L(t, \boldsymbol{x})=\exp (t H) \exp (\boldsymbol{x} \cdot H)$, we find

$$
L(t, \boldsymbol{x})^{-1} d L(t, \boldsymbol{x})=H d t+(1+\gamma) x^{a} P_{a}-\chi \epsilon_{a b} x^{a} P_{b}+\gamma x^{a} B_{a}-\chi \epsilon_{a b} x^{a} B_{b}+d x^{a} P_{a},
$$

so that the soldering form has components

$$
\theta^{H}=d t \quad \text { and } \quad \theta^{P_{a}}=d x^{a}+f^{a}(x) d t,
$$

where $f^{a}(x):=(1+\gamma) x^{a}+\chi \epsilon_{a b} x^{b}$. The vielbein has components

$$
E_{H}=\frac{\partial}{\partial t}-f^{a}(x) \frac{\partial}{\partial x^{a}} \quad \text { and } \quad E_{P_{a}}=\frac{\partial}{\partial x^{a}} .
$$


Therefore, the invariant galilean structure has clock one-form

$$
\eta(\theta)=d t
$$

and inverse spatial metric

$$
\delta^{a b} E_{P_{a}} \otimes E_{P_{b}}=\delta^{a b} \frac{\partial}{\partial x^{a}} \otimes \frac{\partial}{\partial x^{b}}
$$

\section{B Conformal Killing vectors in low dimension}

In this appendix we collect some results concerning the conformal Killing vectors of euclidean space $\mathbb{E}^{D}$, round sphere $\mathbb{S}^{D}$ and hyperbolic space $\mathbb{H}^{D}$ for $D \leq 2$. We have used these results in determining the infinitesimal (conformal) symmetries of the carrollian spacetimes.

For $D=1$, every smooth vector field is conformal Killing. For example, the "metric" on $\mathbb{E}^{1}$ is given by $g=d x^{2}$ relative to the global coordinate $x$. Since the tangent bundle is trivial, we may identify smooth vector fields with smooth functions globally, so $\xi=f(x) \frac{d}{d x}$ for some $f \in C^{\infty}(\mathbb{R})$. Then we see that $\mathcal{L}_{\xi} g=2 d f d x=2 f^{\prime} g$. Similar considerations apply to $\mathbb{S}^{1}$ and $\mathbb{H}^{1}$, with conformal Killing vector fields being in bijective correspondence with the smooth functions $C^{\infty}\left(\mathbb{S}^{1}\right)$ and $C^{\infty}(\mathbb{R})$, respectively.

In all cases, the Lie algebra of conformal Killing vector fields is isomorphic to the Lie algebra of smooth functions under the wronskian Lie bracket:

$$
[f, g]=f g^{\prime}-g f^{\prime}
$$

Things are more interesting for $D=2$. Let us first consider euclidean space with metric $g=d x^{2}+d y^{2}$ relative to global coordinates $(x, y)$. Every vector field is of the form $\xi=u(x, y) \frac{\partial}{\partial x}+v(x, y) \frac{\partial}{\partial y}$ for $u, v \in C^{\infty}\left(\mathbb{R}^{2}\right)$. Then the conformal Killing condition

$$
\mathcal{L}_{\xi} g=2 \frac{\partial u}{\partial x} d x^{2}+2 \frac{\partial u}{\partial y} d y^{2}+2\left(\frac{\partial u}{\partial y}+\frac{\partial v}{\partial x}\right) d x d y \stackrel{!}{=} \lambda\left(d x^{2}+d y^{2}\right)
$$

is equivalent to

$$
\frac{\partial u}{\partial y}+\frac{\partial v}{\partial x}=0 \quad \text { and } \quad \frac{\partial u}{\partial x}=\frac{\partial v}{\partial y}=\frac{\lambda}{2} .
$$

This says that $u$ and $v$ obey the Cauchy-Riemann equations and, since they are smooth, that $w=u(x, y)+i v(x, y)$ is a holomorphic function $f(z)$, say, of $z=x+i y$. In other words, every conformal Killing vector field on $\mathbb{E}^{2}$ is given by

$$
\xi=f(z) \partial+\overline{f(z)} \bar{\partial}
$$

for some entire function $f: \mathbb{C} \rightarrow \mathbb{C}$. The Lie algebra of conformal Killing vector fields on $\mathbb{E}^{2}$ is therefore isomorphic to the Lie algebra $\mathscr{O}(\mathbb{C})$ of entire functions relative to the "wronskian" Lie bracket:

$$
[f, g]=f \partial g-g \partial f
$$

The round sphere $\mathbb{S}^{2}$ is the one-point compactification of $\mathbb{E}^{2}$. A conformal Killing vector field on $\mathbb{S}^{2}$ takes the form $\xi=f(z) \partial+\overline{f(z)} \bar{\partial}$ away from the North pole, say. But demanding 
that $f(z) \partial$ extends to a holomorphic vector field at the North Pole, says that if $\zeta=1 / z$, then $-\zeta^{2} f(1 / \zeta)$ should be holomorphic at $\zeta=0$ and this requires $f(z)=a_{0}+a_{1} z+a_{2} z^{2}$, for some $a_{0}, a_{1}, a_{2} \in \mathbb{C}$. This is the well-known result that the (everywhere smooth) conformal Killing vector fields on $\mathbb{S}^{2}$ define a real Lie algebra isomorphic to $\mathfrak{s l}(2, \mathbb{C}) \cong \mathfrak{s o}(3,1)$. Indeed, the wronskian Lie bracket of the polynomials of degree $\leq 2$ is given by

$$
[1, z]=1, \quad\left[1, z^{2}\right]=2 z \quad \text { and } \quad\left[z, z^{2}\right]=z^{2} .
$$

Finally, let us consider hyperbolic space $\mathbb{H}^{2}$, which we model as the upper half-plane $\left\{(x, y) \in \mathbb{R}^{2} \mid y>0\right\}$ with metric

$$
g=\frac{d x^{2}+d y^{2}}{y^{2}}
$$

The tangent bundle is trivial so that we can write any smooth vector field as $\xi=u(x, y) \frac{\partial}{\partial x}+$ $v(x, y) \frac{\partial}{\partial y}$ for some $u, v \in C^{\infty}\left(\mathbb{R}^{2}\right)$. The conformal Killing condition

$$
\mathcal{L}_{\xi} g=\frac{2}{y^{2}}\left(\frac{\partial u}{\partial y}+\frac{\partial v}{\partial x}\right) d x d y+\frac{2}{y^{2}}\left(\frac{\partial u}{\partial x}-\frac{v}{y}\right) d x^{2}+\frac{2}{y^{2}}\left(\frac{\partial v}{\partial y}-\frac{v}{y}\right) d y^{2} \stackrel{!}{=} \lambda \frac{d x^{2}+d y^{2}}{y^{2}}
$$

results in

$$
\frac{\partial u}{\partial y}+\frac{\partial v}{\partial x}=0 \quad \text { and } \quad \frac{\partial u}{\partial x}=\frac{\partial v}{\partial y}=\frac{\lambda}{2}+\frac{v}{y}
$$

In particular, $u, v$ satisfy the Cauchy-Riemann equations and hence again $w=u+i v=f(z)$, where $f$ is a holomorphic function of $z=x+i y$ in the upper half-plane. The Schwarz reflection principle says that if $f$ extends continuously to $y=0$ then it extends to an entire function on the whole complex plane such that $f(z)=\overline{f(\bar{z})}$ for $z$ in the lower half-plane. But of course $f$ may develop singularities as $y \rightarrow 0$ and hence there are more holomorphic functions on the upper half-plane than can be obtained by restricting entire functions.

In summary, the Lie algebra of conformal Killing vector fields is isomorphic to the Lie algebra $\mathscr{O}(H)$ of holomorphic functions on the upper half-plane relative to the "wronskian" Lie bracket $[f, g]=f \partial g-g \partial f$.

Open Access. This article is distributed under the terms of the Creative Commons Attribution License (CC-BY 4.0), which permits any use, distribution and reproduction in any medium, provided the original author(s) and source are credited.

\section{References}

[1] H. Bacry and J. Levy-Leblond, Possible kinematics, J. Math. Phys. 9 (1968) 1605 [InSPIRE].

[2] J.M. Maldacena, The large $N$ limit of superconformal field theories and supergravity, Int. J. Theor. Phys. 38 (1999) 1113 [hep-th/9711200] [INSPIRE].

[3] C. Duval, G.W. Gibbons and P.A. Horvathy, Conformal Carroll groups and BMS symmetry, Class. Quant. Grav. 31 (2014) 092001 [arXiv: 1402 .5894] [INSPIRE].

[4] A. Strominger, Lectures on the infrared structure of gravity and gauge theory, arXiv: 1703.05448 [INSPIRE]. 
[5] A. Ashtekar, M. Campiglia and A. Laddha, Null infinity, the BMS group and infrared issues, Gen. Rel. Grav. 50 (2018) 140 [arXiv:1808.07093] [InSPIRE].

[6] J. Figueroa-O'Farrill and S. Prohazka, Spatially isotropic homogeneous spacetimes, JHEP 01 (2019) 229 [arXiv: 1809.01224] [INSPIRE].

[7] K. Morand, Embedding Galilean and Carrollian geometries I. Gravitational waves, arXiv:1811.12681 [INSPIRE].

[8] A. Farahmand Parsa, H.R. Safari and M.M. Sheikh-Jabbari, On rigidity of $3 d$ asymptotic symmetry algebras, JHEP 03 (2019) 143 [arXiv: 1809.08209] [INSPIRE].

[9] T. Harmark et al., Strings with non-relativistic conformal symmetry and limits of the AdS/CFT correspondence, JHEP 11 (2018) 190 [arXiv:1810.05560] [INSPIRE].

[10] C. Batlle, J. Gomis, S. Ray and J. Zanelli, Lie symmetries of nonrelativistic and relativistic motions, Phys. Rev. D 99 (2019) 064015 [arXiv: 1812.05837] [InSPIRE].

[11] A. Campoleoni et al., Two-dimensional fluids and their holographic duals, Nucl. Phys. B (2019) 114692 [arXiv:1812.04019] [InSPIRE].

[12] A. Bagchi, A. Mehra and P. Nandi, Field theories with conformal Carrollian symmetry, JHEP 05 (2019) 108 [arXiv: 1901.10147] [INSPIRE].

[13] H.R. Safari and M.M. Sheikh-Jabbari, BMS algebra, its stability and deformations, JHEP 04 (2019) 068 [arXiv: 1902.03260] [INSPIRE].

[14] G.W. Gibbons, The Ashtekar-Hansen universal structure at spatial infinity is weakly pseudo-Carrollian, arXiv: 1902.09170 [INSPIRE].

[15] N. Ozdemir, M. Ozkan, O. Tunca and U. Zorba, Three-dimensional extended newtonian (super)gravity, JHEP 05 (2019) 130 [arXiv:1903.09377] [INSPIRE].

[16] D. Hansen, J. Hartong and N.A. Obers, Gravity between Newton and Einstein, arXiv: 1904.05706 [INSPIRE].

[17] E. Bergshoeff, J.M. Izquierdo, T. Ortín and L. Romano, Lie algebra expansions and actions for non-relativistic gravity, JHEP 08 (2019) 048 [arXiv: 1904.08304] [INSPIRE].

[18] H. Bacry and J. Nuyts, Classification of ten-dimensional kinematical groups with space isotropy, J. Math. Phys. 27 (1986) 2455.

[19] J.M. Figueroa-O'Farrill, Kinematical Lie algebras via deformation theory, J. Math. Phys. 59 (2018) 061701 [arXiv:1711.06111] [INSPIRE].

[20] J.M. Figueroa-O'Farrill, Higher-dimensional kinematical Lie algebras via deformation theory, J. Math. Phys. 59 (2018) 061702 [arXiv:1711.07363] [InSPIRE].

[21] T. Andrzejewski and J.M. Figueroa-O'Farrill, Kinematical Lie algebras in $2+1$ dimensions, J. Math. Phys. 59 (2018) 061703 [arXiv:1802.04048] [InSPIRE].

[22] S. Schäfer-Nameki, M. Yamazaki and K. Yoshida, Coset construction for duals of non-relativistic CFTs, JHEP 05 (2009) 038 [arXiv: 0903.4245] [INSPIRE].

[23] J.I. Jottar, R.G. Leigh, D. Minic and L.A. Pando Zayas, Aging and holography, JHEP 11 (2010) 034 [arXiv: 1004.3752] [INSPIRE].

[24] A. Bagchi and A. Kundu, Metrics with Galilean conformal isometry, Phys. Rev. D 83 (2011) 066018 [arXiv: 1011.4999] [INSPIRE].

[25] C. Duval and S. Lazzarini, Schrödinger manifolds, J. Phys. A 45 (2012) 395203 [arXiv: 1201.0683] [INSPIRE].

[26] E. Bergshoeff, J. Gomis and L. Parra, The symmetries of the Carroll superparticle, J. Phys. A 49 (2016) 185402 [arXiv: 1503.06083] [InSPIRE]. 
[27] X. Bekaert and K. Morand, Connections and dynamical trajectories in generalised Newton-Cartan gravity I. An intrinsic view, J. Math. Phys. 57 (2016) 022507 [arXiv: 1412.8212] [INSPIRE].

[28] K.T. Grosvenor, J. Hartong, C. Keeler and N.A. Obers, Homogeneous nonrelativistic geometries as coset spaces, Class. Quant. Grav. 35 (2018) 175007 [arXiv:1712.03980] [INSPIRE].

[29] C. Duval, G.W. Gibbons and P.A. Horvathy, Conformal Carroll groups, J. Phys. A 47 (2014) 335204 [arXiv: 1403.4213] [INSPIRE].

[30] Y. Voglaire, Strongly exponential symmetric spaces, Int. Math. Res. Not. IMRN 21 (2014) 5974 [arXiv: 1303. 5925].

[31] P.K. Rozanov, On the exponentiality of affine symmetric spaces, Funkt. Anal. i Prilozhen. 43 (2009) 68.

[32] W. Rossmann, Lie groups. An introduction through linear groups, Oxford Graduate Texts in Mathematics volume 5, Oxford University Press, Oxford U.K. (2002).

[33] K. Nomizu, Invariant affine connections on homogeneous spaces, Amer. J. Math. 76 (1954) 33.

[34] C. Duval, G.W. Gibbons, P.A. Horvathy and P.M. Zhang, Carroll versus Newton and Galilei: two dual non-Einsteinian concepts of time, Class. Quant. Grav. 31 (2014) 085016 [arXiv: 1402.0657] [INSPIRE].

[35] H. Bondi, M.G.J. van der Burg and A.W.K. Metzner, Gravitational waves in general relativity. 7. Waves from axisymmetric isolated systems, Proc. Roy. Soc. Lond. A 269 (1962) 21 [INSPIRE].

[36] R. Sachs, Asymptotic symmetries in gravitational theory, Phys. Rev. 128 (1962) 2851 [INSPIRE].

[37] C. Duval, On Galileian isometries, Class. Quant. Grav. 10 (1993) 2217 [arXiv:0903.1641] [INSPIRE].

[38] G. Barnich and G. Compere, Classical central extension for asymptotic symmetries at null infinity in three spacetime dimensions, Class. Quant. Grav. 24 (2007) F15 [gr-qc/0610130] [INSPIRE].

[39] G. Barnich and C. Troessaert, Symmetries of asymptotically flat 4 dimensional spacetimes at null infinity revisited, Phys. Rev. Lett. 105 (2010) 111103 [arXiv:0909.2617] [INSPIRE].

[40] G. Barnich and C. Troessaert, Aspects of the BMS/CFT correspondence, JHEP 05 (2010) 062 [arXiv: 1001.1541] [INSPIRE].

[41] T. Banks, A critique of pure string theory: Heterodox opinions of diverse dimensions, hep-th/0306074 [INSPIRE].

[42] A. Ashtekar, J. Bicak and B.G. Schmidt, Asymptotic structure of symmetry reduced general relativity, Phys. Rev. D 55 (1997) 669 [gr-qc/9608042] [InSPIRE].

[43] C. Duval and P.A. Horvathy, Non-relativistic conformal symmetries and Newton-Cartan structures, J. Phys. A 42 (2009) 465206 [arXiv:0904.0531] [InSPIRE].

[44] J. Matulich, S. Prohazka and J. Salzer, Limits of three-dimensional gravity and metric kinematical Lie algebras in any dimension, JHEP 07 (2019) 118 [arXiv:1903.09165] [INSPIRE]. 